\title{
Rotating Einstein-Yang-Mills Black Holes
}

\author{
Burkhard Kleihaus \\ Department of Mathematical Physics, University College, Dublin, \\ Belfield, Dublin 4, Ireland \\ Jutta Kunz and Francisco Navarro-Lérida ${ }^{1}$ \\ Fachbereich Physik, Universität Oldenburg, Postfach 2503 \\ D-26111 Oldenburg, Germany
}

November 8, 2018

\begin{abstract}
We construct rotating hairy black holes in SU(2) Einstein-Yang-Mills theory. These stationary axially symmetric black holes are asymptotically flat. They possess non-trivial non-Abelian gauge fields outside their regular event horizon, and they carry non-Abelian electric charge. In the limit of vanishing angular momentum, they emerge from the neutral static spherically symmetric EinsteinYang-Mills black holes, labelled by the node number of the gauge field function. With increasing angular momentum and mass, the non-Abelian electric charge of the solutions increases, but remains finite. The asymptotic expansion for these black hole solutions includes non-integer powers of the radial variable.
\end{abstract}

1 Dept. de Física Teórica II, Ciencias Físicas, Universidad Complutense de Madrid, E-28040 Madrid, Spain 


\section{Introduction}

The unique family of stationary asymptotically flat black holes of Einstein-Maxwell (EM) theory comprises the rotating Kerr-Newman and Kerr black holes and the static Reissner-Nordstrøm and Schwarzschild black holes. EM black holes are completely determined by their mass, their charge and their angular momentum, i.e. EM black holes have "no hair" [1, 2].

The EM "no-hair" theorem does not generalize to theories with non-Abelian gauge fields coupled to gravity [3]. The generic black hole solutions of SU(2) Einstein-YangMills (EYM) theory possess non-trivial magnetic fields outside their regular event horizon, representing non-Abelian "hair" [3]. Besides static spherically symmetric hairy black holes [4], there are also static hairy black holes, which are not spherically but only axially symmetric [5, 6, 7]. This shows that Israel's theorem neither generalizes to theories with non-Abelian gauge fields coupled to gravity.

Obviously, also hairy stationary black hole solutions, representing the non-Abelian generalizations of the Kerr-Newman black hole solutions, should exist, as conjectured long ago [8]. The construction of such hairy rotating black hole solutions, however, appeared very difficult. First of all, it was not clear, whether the standard LewisPapapetrou parametrization of the stationary axially symmetric metric [9] would be sufficiently general and whether an ansatz for the gauge fields, satisfying the Ricci circularity and Frobenius conditions [9, 10], was available [11, 12, 13]. Second, even within the standard metric parametrization the black hole solutions would only possess axial symmetry. Therefore the construction of such solutions would involve the solution of a large system of coupled non-linear partial differential equations for the metric and gauge field functions and thus represent a numerical challenge.

First progress was achieved with the construction of perturbative stationary nonAbelian black hole solutions in SU(2) EYM theory [12]. Based on the static hairy black hole solutions, these slowly rotating black hole solutions revealed an unexpected property. The rotating black holes carry non-Abelian electric charge, whereas their static counterparts are electrically neutral [12]. In the static spherically symmetric case electrically charged $\mathrm{SU}(2)$ black holes are even prohibited by the 'non-Abelian baldness' theorem [14]. Indeed, the non-Abelian electric charge of the slowly rotating black hole solutions turned out to be proportional to their angular momentum, and thus vanishes in the static limit. Subsequently, perturbative calculations with more general boundary conditions predicted even more exotic stationary hairy black hole solutions [15].

Only recently non-pertubative rotating hairy black hole solutions were obtained

in $\mathrm{SU}(2)$ EYM theory [16], confirming the perturbative calculations [12]. They are obtained within the standard Lewis-Papapetrou parametrization of the metric, and the ansatz for the gauge fields is consistent with the circularity and Frobenius conditions. 
Representing the first set of non-perturbative rotating hairy black hole solutions, they possess three global charges, a mass, an angular momentum, and a small non-Abelian electric charge. They do not carry non-Abelian magnetic charge, although they possess non-trivial magnetic gauge fields outside their regular event horizon.

Here we present a detailed account of these rotating hairy black holes, announced in [16. We analyze their properties. In particular we discuss their global charges and their horizon charges [17]. We further introduce local charges, to illustrate the contributions of the gauge fields outside the event horizon. The black hole solutions depend on two continuous parameters, the horizon size, and the angular velocity of the horizon. Like their static spherically symmetric counterparts, they further depend on the integer node number $k$ of the gauge field functions. Thus for a given horizon size, when a small angular velocity of the horizon is imposed, a sequence of rotating black hole solutions, labelled by the node number $k$, emerges from the sequence of static black hole solutions.

Furthermore, we present the expansions of the metric and gauge field functions at the horizon and at infinity for these black holes. The asymptotic expansion for these black holes has the surprising feature, that the magnetic gauge field functions approach their asymptotic values with non-integer powers of the radial coordinate. In particular, the non-integer powers depend on the non-Abelian electric charge. In the static limit, the non-Abelian charge vanishes and the well-known integer power fall-off of the static spherically symmetric functions is recovered.

In section 2 we recall the SU(2) EYM action and the equations of motion. We present the stationary ansatz for the metric and the gauge field, and discuss its residual $\mathrm{U}(1)$ gauge invariance. The global properties of the black hole solutions and their horizon properties are presented in section 3. They are obtained from the expansions at infinity and at the horizon. The expansions also suggest the set of boundary conditions to be satisfied by the solutions at the horizon and at infinity. The boundary conditions along the axes, follow from symmetry and regularity conditions. For comparison, we present in section 4 the embedded Kerr-Newman solutions. In particular, we transform the Kerr-Newman solutions from Boyer-Lindquist to isotropic coordinates, and further transform them to the gauge employed for the non-Abelian solutions. Our numerical results are discussed in section 5. In section 6 we present our conclusions. Appendix A demonstrates the circularity condition, Appendix $\mathrm{B}$ and $\mathrm{C}$ give details of the expansion at infinity and at the origin, respectively.

\section{$2 \mathrm{SU}(2)$ EYM action and stationary Ansatz}

We here briefly recall the SU(2) EYM action and the general set of EYM equations to be satisfied by the stationary hairy black hole solutions. We then discuss the ansatz for the metric and the gauge field functions [16]. The metric chosen is the stationary 
axially symmetric Lewis-Papapetrou metric [9] in isotropic coordinates. The ansatz for the gauge field represents a generalization of the previously employed static axially symmetric parameterization [18, 5] to the stationary case, satisfying the Ricci circularity and Frobenius conditions [9].

\section{$2.1 \quad \mathrm{SU}(2)$ EYM equations}

We consider the SU(2) Einstein-Yang-Mills action

$$
S=\int\left(\frac{R}{16 \pi G}-\frac{1}{2} \operatorname{Tr}\left(F_{\mu \nu} F^{\mu \nu}\right)\right) \sqrt{-g} d^{4} x
$$

with curvature scalar $R$, Newton's constant $G$, and $\mathrm{SU}(2)$ field strength tensor

$$
F_{\mu \nu}=\partial_{\mu} A_{\nu}-\partial_{\nu} A_{\mu}+i e\left[A_{\mu}, A_{\nu}\right]
$$

where $A_{\mu}$ denotes the gauge fields

$$
A_{\mu}=\frac{1}{2} \tau^{a} A_{\mu}^{a}
$$

and $e$ the Yang-Mills coupling constant. The gauge fields transform as

$$
A_{\mu}^{\prime}=U A_{\mu} U^{\dagger}+\frac{i}{e}\left(\partial_{\mu} U\right) U^{\dagger}
$$

under $\mathrm{SU}(2)$ gauge transformations $U$.

Variation of the action (1) with respect to the metric $g^{\mu \nu}$ leads to the Einstein equations

$$
G_{\mu \nu}=R_{\mu \nu}-\frac{1}{2} g_{\mu \nu} R=8 \pi G T_{\mu \nu}
$$

with stress-energy tensor

$$
\begin{aligned}
T_{\mu \nu} & =g_{\mu \nu} L_{M}-2 \frac{\partial L_{M}}{\partial g^{\mu \nu}} \\
& =2 \operatorname{Tr}\left(F_{\mu \alpha} F_{\nu \beta} g^{\alpha \beta}-\frac{1}{4} g_{\mu \nu} F_{\alpha \beta} F^{\alpha \beta}\right),
\end{aligned}
$$

variation with respect to the gauge field $A_{\mu}$ leads to the matter field equations,

$$
\frac{1}{\sqrt{-g}} D_{\mu}\left(\sqrt{-g} F^{\mu \nu}\right)=0
$$




\subsection{Stationary ansatz for the metric}

To construct rotating axially symmetric EYM black hole solutions, we employ isotropic coordinates for the metric. In terms of spherical coordinates $r, \theta$ and $\varphi$ the LewisPapapetrou metric is parameterized as 16]

$$
d s^{2}=-f d t^{2}+\frac{m}{f} d r^{2}+\frac{m r^{2}}{f} d \theta^{2}+\frac{l r^{2} \sin ^{2} \theta}{f}\left(d \varphi+\frac{\omega}{r} d t\right)^{2},
$$

where the four metric functions $f, m, l$ and $\omega$ depend only on the coordinates $r$ and $\theta$. The $z$-axis represents the symmetry axis.

The metric has Killing vector fields $\xi=\partial_{t}$ and $\eta=\partial_{\varphi}$. The regularity condition along the $z$-axis [9],

$$
\frac{X,{ }_{\mu} X^{, \mu}}{4 X} \longrightarrow 1, \quad X=\eta^{\mu} \eta_{\mu}
$$

requires

$$
\left.m\right|_{\theta=0}=\left.l\right|_{\theta=0} .
$$

The ansatz for the metric, Eq. (8), satisfies the Ricci circularity conditions [9, 10, 2]

$$
\xi^{\mu} R_{\mu[\alpha} \xi_{\beta} \eta_{\gamma]}=0=\eta^{\mu} R_{\mu[\alpha} \xi_{\beta} \eta_{\gamma]}
$$

where $R_{\mu \nu}$ is the Ricci tensor, and the Frobenius conditions

$$
\xi_{[\mu} \eta_{\nu} \eta_{\lambda] ; \tau}=0=\eta_{[\mu} \xi_{\nu} \xi_{\lambda] ; \tau}
$$

implying the corresponding conditions for the stress-energy tensor for solutions of the EYM equations (see section 2.3.2 and Appendix A).

The event horizon of stationary black hole solutions resides at a surface of constant radial coordinate, $r=r_{\mathrm{H}}$, and is characterized by the condition $f\left(r_{\mathrm{H}}\right)=0$ [16]. The Killing vector field

$$
\chi=\partial_{t}-\omega_{\mathrm{H}} / r_{\mathrm{H}} \partial_{\varphi}
$$

is orthogonal to and null on the horizon [19.

The ergosphere, defined as the region in which $\xi_{\mu} \xi^{\mu}$ is positive, is bounded by the event horizon and by the surface where

$$
-f+\sin ^{2} \theta \frac{l}{f} \omega^{2}=0
$$




\subsection{Stationary ansatz for the gauge field}

For the gauge field $A_{\mu}$ we choose the ansatz [16]

$$
A_{\mu} d x^{\mu}=\Psi d t+A_{\varphi}\left(d \varphi+\frac{\omega}{r} d t\right)+\left(\frac{H_{1}}{r} d r+\left(1-H_{2}\right) d \theta\right) \frac{\tau_{\varphi}}{2 e}
$$

with

$$
\Psi=B_{1} \frac{\tau_{r}}{2 e}+B_{2} \frac{\tau_{\theta}}{2 e}
$$

and

$$
A_{\varphi}=-\sin \theta\left[H_{3} \frac{\tau_{r}}{2 e}+\left(1-H_{4}\right) \frac{\tau_{\theta}}{2 e}\right] .
$$

Here the symbols $\tau_{r}, \tau_{\theta}$ and $\tau_{\varphi}$ denote the dot products of the Cartesian vector of Pauli matrices, $\vec{\tau}=\left(\tau_{x}, \tau_{y}, \tau_{z}\right)$, with the spherical spatial unit vectors,

$$
\begin{gathered}
\vec{e}_{r}=(\sin \theta \cos \varphi, \sin \theta \sin \varphi, \cos \theta), \\
\vec{e}_{\theta}=(\cos \theta \cos \varphi, \cos \theta \sin \varphi,-\sin \theta), \\
\vec{e}_{\varphi}=(-\sin \varphi, \cos \varphi, 0) .
\end{gathered}
$$

The two electric gauge field functions $B_{i}$ and the four magnetic gauge field functions $H_{i}$ depend only on the coordinates $r$ and $\theta$.

In the static limit, the ansatz reduces to the static spherically symmetric $\mathrm{SU}(2)$ EYM ansatz, where

$$
l=m, \quad \omega=0, \quad H_{2}=H_{4}, \quad H_{1}=H_{3}=B_{1}=B_{2}=0,
$$

and all non-trivial functions depend only on the radial coordinate $r$.

\subsubsection{Residual gauge invariance}

The ansatz (15)-(17) is axially symmetric in the sense, that a rotation around the symmetry axis can be compensated by a gauge rotation. The ansatz is form-invariant under Abelian gauge transformations where [18, 0, 0, 16]

$$
U=\exp \left(\frac{i}{2} \tau_{\varphi} \Gamma(r, \theta)\right)
$$

The functions $H_{1}$ and $H_{2}$ transform inhomogeneously under such gauge transformations,

$$
\begin{aligned}
& H_{1} \rightarrow H_{1}-r \partial_{r} \Gamma \\
& H_{2} \rightarrow H_{2}+\partial_{\theta} \Gamma
\end{aligned}
$$


like a 2-dimensional gauge field. The functions $H_{3}$ and $H_{4}$ combine to form a scalar doublet, $\left(H_{3}+\cot \theta,-H_{4}\right)$,

$$
\begin{aligned}
H_{3}+\cot \theta & \rightarrow \cos \Gamma\left(H_{3}+\cot \theta\right)+\sin \Gamma\left(-H_{4}\right), \\
-H_{4} & \rightarrow \cos \Gamma\left(-H_{4}\right)-\sin \Gamma\left(H_{3}+\cot \theta\right) .
\end{aligned}
$$

Similarly, the functions $B_{1}$ and $B_{2}$ transform as

$$
\begin{aligned}
& B_{1}+\cos \theta \frac{\omega}{r} \rightarrow \cos \Gamma\left(B_{1}+\cos \theta \frac{\omega}{r}\right)+\sin \Gamma\left(B_{2}-\sin \theta \frac{\omega}{r}\right) \\
& B_{2}-\sin \theta \frac{\omega}{r} \rightarrow \cos \Gamma\left(B_{2}-\sin \theta \frac{\omega}{r}\right)-\sin \Gamma\left(B_{1}+\cos \theta \frac{\omega}{r}\right) .
\end{aligned}
$$

As previously [18, 5, 16], we choose the gauge condition

$$
r \partial_{r} H_{1}-\partial_{\theta} H_{2}=0
$$

with respect to this residual gauge degree of freedom.

\subsubsection{Stress-energy tensor}

Let us now address the stress-energy tensor $T_{\mu \nu}$ and the consistency of the ansatz for the gauge fields, Eqs. (15)-(17), with the Ricci circularity conditions. The stress-energy is circular, when [9, 10, 2]

$$
\xi^{\mu} T_{\mu[\alpha} \xi_{\beta} \eta_{\gamma]}=0=\eta^{\mu} T_{\mu[\alpha} \xi_{\beta} \eta_{\gamma]}
$$

To verify these circularity conditions for the stress-energy tensor $T_{\mu \nu}$, Eq. (6), we expand the field strength tensor in the form

$$
F_{\mu \nu}=\sum_{\alpha} F_{\mu \nu}^{(\alpha)} \frac{\tau_{\alpha}}{2 e}, \quad \alpha=r, \theta, \varphi
$$

where its non-vanishing components $F_{\mu \nu}^{(\alpha)}$ are given by

$$
\begin{aligned}
F_{r \theta}^{(\varphi)} & =-\frac{1}{r}\left[H_{1, \theta}+r H_{2, r}\right], \\
F_{r \varphi}^{(r)} & =-\frac{\sin \theta}{r}\left[r H_{3, r}-H_{1} H_{4}\right], \\
F_{r \varphi}^{(\theta)} & =\frac{\sin \theta}{r}\left[r H_{4, r}+H_{1}\left(H_{3}+\cot \theta\right)\right], \\
F_{\theta \varphi}^{(r)} & =-\sin \theta\left[H_{3, \theta}+H_{3} \cot \theta+H_{2} H_{4}-1\right], \\
F_{\theta \varphi}^{(\theta)} & =\sin \theta\left[H_{4, \theta}+\cot \theta\left(H_{4}-H_{2}\right)-H_{2} H_{3}\right],
\end{aligned}
$$




$$
\begin{aligned}
F_{t r}^{(r)} & =-\frac{1}{r}\left[r B_{1, r}+H_{1} B_{2}-\frac{\omega}{r} \sin \theta\left(H_{1}\left(1-H_{4}\right)-H_{3}+r H_{3, r}\right)-\omega_{, r} \sin \theta H_{3}\right], \\
F_{t r}^{(\theta)} & =-\frac{1}{r}\left[r B_{2, r}-H_{1} B_{1}+\frac{\omega}{r} \sin \theta\left(H_{1} H_{3}+\left(1-H_{4}\right)+r H_{4, r}\right)-\omega_{, r} \sin \theta\left(1-H_{4}\right)\right], \\
F_{t \theta}^{(r)} & =-\left[B_{1, \theta}-H_{2} B_{2}+\frac{\omega}{r} \sin \theta\left(H_{2}\left(1-H_{4}\right)-\cot \theta H_{3}-H_{3, \theta}\right)-\frac{\omega_{, \theta}}{r} \sin \theta H_{3}\right], \\
F_{t \theta}^{(\theta)} & =-\left[B_{2, \theta}+H_{2} B_{1}-\frac{\omega}{r} \sin \theta\left(H_{2} H_{3}+\cot \theta\left(1-H_{4}\right)-H_{4, \theta}\right)-\frac{\omega_{, \theta}}{r} \sin \theta\left(1-H_{4}\right)\right], \\
F_{t \varphi}^{(\varphi)} & =-\sin \theta\left[B_{1} H_{4}+B_{2}\left(H_{3}+\cot \theta\right)-\frac{\omega}{r} \sin \theta\left(\cot \theta\left(1-H_{4}\right)+H_{3}\right)\right] .
\end{aligned}
$$

Now it is easily seen, that $T_{t r}=T_{t \theta}=T_{\varphi r}=T_{\varphi \theta}=0$. Thus the circularity conditions are satisfied (see Appendix A).

Of particular interest are also the energy density of the matter fields, $\varepsilon=-T_{0}^{0}$, and the angular momentum density $j=T_{\varphi}^{0}$. They are given by

$$
\begin{aligned}
-T_{0}^{0}= & \frac{1}{2 e^{2} r^{4} m}\left\{\frac{f^{2}}{m}\left(r F_{r \theta}^{(\varphi)}\right)^{2}+\left(\frac{f^{2}}{l \sin ^{2} \theta}-\omega^{2}\right)\left[\left(r F_{r \varphi}^{(r)}\right)^{2}+\left(r F_{r \varphi}^{(\theta)}\right)^{2}+\left(F_{\theta \varphi}^{(r)}\right)^{2}+\left(F_{\theta \varphi}^{(\theta)}\right)^{2}\right]\right. \\
& \left.+r^{2}\left[\left(r F_{t r}^{(r)}\right)^{2}+\left(r F_{t r}^{(\theta)}\right)^{2}+\left(F_{t \theta}^{(r)}\right)^{2}+\left(F_{t \theta}^{(\theta)}\right)^{2}+\frac{m}{l \sin ^{2} \theta}\left(F_{t \varphi}^{(\varphi)}\right)^{2}\right]\right\},
\end{aligned}
$$

and

$$
\begin{aligned}
T_{\varphi}^{0}= & \frac{1}{r^{2} m}\left\{r^{2}\left(F_{r \varphi}^{(r)} F_{t r}^{(r)}+F_{r \varphi}^{(\theta)} F_{t r}^{(\theta)}\right)+F_{\theta \varphi}^{(r)} F_{t \theta}^{(r)}+F_{\theta \varphi}^{(\theta)} F_{t \theta}^{(\theta)}\right. \\
& \left.+\frac{\omega}{r}\left[\left(r F_{r \varphi}^{(r)}\right)^{2}+\left(r F_{r \varphi}^{(\theta)}\right)^{2}+\left(F_{\theta \varphi}^{(r)}\right)^{2}+\left(F_{\theta \varphi}^{(\theta)}\right)^{2}\right]\right\},
\end{aligned}
$$

respectively. As seen from Eqs. (27) and (28), regularity of the energy density on the $z$-axis requires

$$
\left.H_{2}\right|_{\theta=0}=\left.H_{4}\right|_{\theta=0}
$$

\section{Black Hole properties}

To obtain stationary axially symmetric black hole solutions which are asymptotically flat, and possess a regular event horizon, as well as a finite mass, angular momentum and electric charge, we need to impose the appropriate set of boundary conditions. Looking for black hole solutions with parity reflection symmetry, we need to consider the solutions only for $0 \leq \theta \leq \pi / 2$. Thus appropriate boundary conditions must be imposed at infinity and at the horizon, along the $\rho$-axis and along the $z$-axis (i. e. for $\theta=\pi / 2$ and $\theta=0)$.

In the following we present these boundary conditions at infinity, at the horizon, and along the axes. The choice of boundary conditions is consistent with the equations 
of motion, as seen from the expansions of the metric and gauge field functions at infinity and at the horizon. These expansions also allow the extraction of the physical properties of these solutions, such as their global charges or their horizon charges. We also introduce local charges of the black hole solutions.

\subsection{Behaviour at infinity}

\subsubsection{Boundary conditions at infinity}

For notational simplicity we now introduce the dimensionless coordinate $x$,

$$
x=\frac{e}{\sqrt{4 \pi G}} r
$$

and the dimensionless electric gauge field functions $\bar{B}_{1}$ and $\bar{B}_{2}$,

$$
\bar{B}_{1}=\frac{\sqrt{4 \pi G}}{e} B_{1}, \quad \bar{B}_{2}=\frac{\sqrt{4 \pi G}}{e} B_{2} .
$$

To obtain asymptotically flat solutions, we impose on the metric functions at infinity $(x=\infty)$ the boundary conditions

$$
\left.f\right|_{x=\infty}=\left.m\right|_{x=\infty}=\left.l\right|_{x=\infty}=1,\left.\quad \omega\right|_{x=\infty}=0 .
$$

By requiring the four magnetic gauge field functions $H_{i}$ to satisfy

$$
\left.H_{1}\right|_{x=\infty}=\left.H_{3}\right|_{x=\infty}=0,\left.\quad H_{2}\right|_{x=\infty}=\left.H_{4}\right|_{x=\infty}= \pm 1,
$$

and the two electric gauge field functions $\bar{B}_{i}$ to satisfy

$$
\left.\bar{B}_{1}\right|_{x=\infty}=\left.\bar{B}_{2}\right|_{x=\infty}=0
$$

the black hole solutions are magnetically neutral, but they may carry a non-Abelian electric charge.

The node number $k$ of the gauge field functions is defined by the number of nodes of the functions $H_{2}$ and $H_{4}$ [18, 5]. For each node number there are two degenerate solutions, specified by the value of $H_{2}$ and $H_{4}$ at infinity, $H_{2}(\infty)=H_{4}(\infty)= \pm 1$. These solutions are related by the large gauge transformation $U=\tau_{r}$, transforming the gauge field functions according to

$$
\begin{gathered}
H_{1} \rightarrow-H_{1}, \quad H_{2} \rightarrow-H_{2}, \quad H_{3} \rightarrow+H_{3}, \quad H_{4} \rightarrow-H_{4} \\
\bar{B}_{1} \rightarrow+\bar{B}_{1}, \quad \bar{B}_{2} \rightarrow-\bar{B}_{2}+\sin \theta \frac{\omega}{x}
\end{gathered}
$$

Because of this gauge symmetry we can choose the gauge field functions to satisfy $H_{2}(\infty)=H_{4}(\infty)=-1$. Solutions with an odd number of nodes then have positive values of $\mathrm{H}_{2}$ and $\mathrm{H}_{4}$ at the horizon, whereas solutions with an even number of nodes have negative values of $H_{2}$ and $H_{4}$ at the horizon. 


\subsubsection{Expansion at infinity}

The asymptotic expressions for the metric and gauge field functions are

$$
\begin{aligned}
& f=1-\frac{2 M}{x}+O\left(\frac{1}{x^{2}}\right), \\
& m=1+\frac{C_{1}}{x^{2}}+\frac{Q^{2}-M^{2}-2 C_{1}}{x^{2}} \sin ^{2} \theta+o\left(\frac{1}{x^{2}}\right), \\
& l=1+\frac{C_{1}}{x^{2}}+o\left(\frac{1}{x^{2}}\right) \\
& \omega=-\frac{2 a M}{x^{2}}+O\left(\frac{1}{x^{3}}\right) \\
& H_{1}=\left[\frac{2 C_{5}}{x^{2}}+\frac{8 C_{4}}{\beta-1} x^{-\frac{1}{2}(\beta-1)}-\frac{2 C_{2} C_{3}(\alpha+3)}{(\alpha+5) Q^{2}} x^{-\frac{1}{2}(\alpha+1)}\right] \sin \theta \cos \theta+o\left(\frac{1}{x^{2}}\right) \\
& H_{2}=-1+C_{3} x^{-\frac{1}{2}(\alpha-1)}+o\left(x^{-\frac{1}{2}(\alpha-1)}\right), \\
& H_{3}=\left(\frac{C_{2}}{x}+C_{3} x^{-\frac{1}{2}(\alpha-1)}\right) \sin \theta \cos \theta+o\left(\frac{1}{x}\right), \\
& H_{4}=-1+C_{3} x^{-\frac{1}{2}(\alpha-1)}-\left(\frac{C_{2}}{x}+C_{3} x^{-\frac{1}{2}(\alpha-1)}\right) \sin ^{2} \theta+o\left(\frac{1}{x}\right), \\
& \bar{B}_{1}=\frac{Q \cos \theta}{x}+O\left(\frac{1}{x^{2}}\right) \\
& \bar{B}_{2}=\frac{Q \sin \theta}{x}+O\left(\frac{1}{x^{2}}\right),
\end{aligned}
$$

where $a, M, Q$, and $C_{1}, \ldots, C_{5}$ are dimensionless constants, and $\alpha$ and $\beta$ determine the non-integer fall-off of the magnetic gauge field functions $H_{i}$, with

$$
\alpha=\sqrt{9-4 Q^{2}}, \quad \beta=\sqrt{25-4 Q^{2}} .
$$

Further details of the asymptotic expansion are presented in Appendix B. 


\subsubsection{Global charges}

Let us now obtain the global charges of the EYM solutions from the asymptotic behaviour of the metric and gauge field functions.

The mass and the angular momentum are obtained from the metric components $g_{t t}$ and $g_{t \varphi}$, respectively [20]. The asymptotic expansions for the metric functions $f$ and $\omega$, Eqs. (36) and (39), yield for the dimensionless mass $M$ and the dimensionless angular momentum $J=a M$ the expressions

$$
M=\frac{1}{2} \lim _{x \rightarrow \infty} x^{2} \partial_{x} f, \quad J=\frac{1}{2} \lim _{x \rightarrow \infty} x^{2} \omega .
$$

The asymptotic behaviour of the gauge fields yields the non-Abelian electric charge $Q^{\mathrm{YM}}$ and magnetic charge $P^{\mathrm{YM}}$ of the black hole solutions. Let us define the gaugeinvariant non-Abelian electric charge $Q^{\mathrm{YM}}$ [17]

$$
Q^{\mathrm{YM}}=\frac{1}{4 \pi} \oint \sqrt{\sum_{i}\left({ }^{*} F_{\theta \varphi}^{i}\right)^{2}} d \theta d \varphi=\frac{Q}{e},
$$

where the integral is evaluated at spatial infinity, and ${ }^{*} F$ represents the dual field strength tensor. Insertion of the asymptotic expansion of the gauge field functions, Eqs. (40)-(45), into the respective field strength tensor components, Eq. (26), then yields for the dimensionless non-Abelian electric charge

$$
Q=\lim _{x \rightarrow \infty} x\left(\cos \theta \bar{B}_{1}+\sin \theta \bar{B}_{2}\right),
$$

i. e. the non-Abelian electric charge can be read off directly from the asymptotic behaviour of the electric gauge field functions $\bar{B}_{1}$ and $\bar{B}_{2}$.

Likewise, the gauge-invariant non-Abelian magnetic charge $P^{\mathrm{YM}}$ is obtained from [17]

$$
P^{\mathrm{YM}}=\frac{1}{4 \pi} \oint \sqrt{\sum_{i}\left(F_{\theta \varphi}^{i}\right)^{2}} d \theta d \varphi=\frac{P}{e},
$$

where the integral is evaluated at spatial infinity. Again, insertion of the asymptotic expansion of the gauge field functions into the respective field strength tensor components yields the global charge. For the dimensionless non-Abelian magnetic charge we thus obtain

$$
P=0 \text {. }
$$

As imposed by the boundary conditions, the EYM solutions carry no magnetic charge. 


\subsection{Boundary conditions along the axes}

The boundary conditions along the $\rho$ - and $z$-axis $(\theta=\pi / 2$ and $\theta=0)$ are determined by the symmetries. The metric functions satisfy along the axes

$$
\begin{aligned}
& \left.\partial_{\theta} f\right|_{\theta=0}=\left.\partial_{\theta} m\right|_{\theta=0}=\left.\partial_{\theta} l\right|_{\theta=0}=\left.\partial_{\theta} \omega\right|_{\theta=0}=0, \\
& \left.\partial_{\theta} f\right|_{\theta=\frac{\pi}{2}}=\left.\partial_{\theta} m\right|_{\theta=\frac{\pi}{2}}=\left.\partial_{\theta} l\right|_{\theta=\frac{\pi}{2}}=\left.\partial_{\theta} \omega\right|_{\theta=\frac{\pi}{2}}=0 .
\end{aligned}
$$

For the gauge field functions symmetry considerations lead to the boundary conditions

$$
\begin{gathered}
\left.H_{1}\right|_{\theta=0}=\left.H_{3}\right|_{\theta=0}=0,\left.\quad \partial_{\theta} H_{2}\right|_{\theta=0}=\left.\partial_{\theta} H_{4}\right|_{\theta=0}=0, \\
\left.\bar{B}_{2}\right|_{\theta=0}=0,\left.\quad \partial_{\theta} \bar{B}_{1}\right|_{\theta=0}=0, \\
\left.H_{1}\right|_{\theta=\frac{\pi}{2}}=\left.H_{3}\right|_{\theta=\frac{\pi}{2}}=0,\left.\quad \partial_{\theta} H_{2}\right|_{\theta=\frac{\pi}{2}}=\left.\partial_{\theta} H_{4}\right|_{\theta=\frac{\pi}{2}}=0, \\
\left.\bar{B}_{1}\right|_{\theta=\frac{\pi}{2}}=0,\left.\quad \partial_{\theta} \bar{B}_{2}\right|_{\theta=\frac{\pi}{2}}=0
\end{gathered}
$$

along the axes. In addition, regularity on the $z$-axis requires condition (10) for the metric functions to be satisfied, and condition (30) for the gauge field functions.

\subsection{Behaviour at the horizon}

\subsubsection{Boundary conditions at the horizon}

The event horizon of stationary black hole solutions resides at a surface of constant radial coordinate, $x=x_{\mathrm{H}}$, and is characterized by the condition $f\left(x_{\mathrm{H}}\right)=0$ [16]. Requiring the horizon to be regular, we obtain the boundary conditions at the horizon.

At a regular horizon the metric functions must satisfy

$$
\left.f\right|_{x=x_{\mathrm{H}}}=\left.m\right|_{x=x_{\mathrm{H}}}=\left.l\right|_{x=x_{\mathrm{H}}}=0,\left.\quad \omega\right|_{x=x_{\mathrm{H}}}=\omega_{\mathrm{H}}
$$

where $\omega_{\mathrm{H}}$ is constant at the horizon.

To obtain the boundary conditions for the gauge field functions, we start by requiring that the electro-static potential $\Psi=\left(\chi^{\mu} A_{\mu}\right)$, with Killing vector $\chi$, Eq. (13), is constant at the horizon [21],

$$
\Psi_{\mathrm{H}}=\left.\left(\chi^{\mu} A_{\mu}\right)\right|_{x=x_{\mathrm{H}}}=\text { const },
$$

(see also Appendix C). This yields the conditions

$$
\begin{aligned}
& \left.\bar{B}_{1}\right|_{x=x_{\mathrm{H}}}=-\cos \theta \Psi_{0}, \\
& \left.\bar{B}_{2}\right|_{x=x_{\mathrm{H}}}=\sin \theta \Psi_{0} .
\end{aligned}
$$


The equations of motion, Eqs. (可), then yield for non-Abelian solutions the conditions

$$
\begin{gathered}
\Psi_{0}=\omega_{\mathrm{H}} / x_{\mathrm{H}}, \\
\left.\partial_{x} \omega\right|_{x=x_{\mathrm{H}}}=\omega_{\mathrm{H}} / x_{\mathrm{H}},
\end{gathered}
$$

as well as

$$
\left.\left(x \partial_{x} \bar{B}_{1}\right)\right|_{x=x_{\mathrm{H}}}=0,\left.\quad\left(x \partial_{x} \bar{B}_{2}\right)\right|_{x=x_{\mathrm{H}}}=0,
$$

and, taking these conditions into account,

$$
\begin{aligned}
\left.\left(\partial_{\theta} H_{1}+x \partial_{x} H_{2}\right)\right|_{x=x_{\mathrm{H}}} & =0, \\
\left.\left(x \partial_{x} H_{3}-H_{1} H_{4}\right)\right|_{x=x_{\mathrm{H}}} & =0, \\
\left.\left(x \partial_{x} H_{4}+H_{1}\left(H_{3}+\cot \theta\right)\right)\right|_{x=x_{\mathrm{H}}} & =0,
\end{aligned}
$$

which derive from $F_{r \theta}^{(\varphi)}, F_{r \phi}^{(r)}$, and $F_{r \phi}^{(\theta)}$, respectively. Thus the regularity conditions at the horizon imply

$$
\left.\chi^{\mu} F_{\mu \nu}\right|_{x=x_{\mathrm{H}}}=0,\left.\quad F_{r \theta}\right|_{x=x_{\mathrm{H}}}=0,\left.\quad F_{r \varphi}\right|_{x=x_{\mathrm{H}}}=0 .
$$

Furthermore, the gauge condition, Eq. (24), implies

$$
\left.\left(x \partial_{x} H_{1}-\partial_{\theta} H_{2}\right)\right|_{x=x_{\mathrm{H}}}=0 .
$$

However, for black hole solutions the gauge condition (24) still allows for non-trivial gauge transformations satisfying

$$
x^{2} \partial_{x}^{2} \Gamma+x \partial_{x} \Gamma+\partial_{\theta}^{2} \Gamma=0 .
$$

To fix the gauge, we choose the additional condition [18, 5, 16]

$$
\left.\left(\partial_{\theta} H_{1}\right)\right|_{x=x_{\mathrm{H}}}=0,
$$

which implies $\left.H_{1}\right|_{x=x_{\mathrm{H}}}=0$, when we take into account the boundary condition on the axes, $\left.H_{1}\right|_{\theta=0, \pi / 2}=0$, Eq. (53).

For the numerical solutions we then impose on the gauge field functions the set of boundary conditions

$$
\begin{gathered}
\left.H_{1}\right|_{x=x_{\mathrm{H}}}=0,\left.\quad \partial_{x} H_{2}\right|_{x=x_{\mathrm{H}}}=0,\left.\quad \partial_{x} H_{3}\right|_{x=x_{\mathrm{H}}}=0,\left.\quad \partial_{x} H_{4}\right|_{x=x_{\mathrm{H}}}=0, \\
\left.x_{\mathrm{H}} \bar{B}_{1}\right|_{x=x_{\mathrm{H}}}=-\cos \theta \omega_{\mathrm{H}},\left.\quad x_{\mathrm{H}} \bar{B}_{2}\right|_{x=x_{\mathrm{H}}}=\sin \theta \omega_{\mathrm{H}} .
\end{gathered}
$$




\subsubsection{Expansion at the horizon}

Expanding the metric and gauge field functions at the horizon in powers of

$$
\delta=\frac{x}{x_{\mathrm{H}}}-1
$$

yields

$$
\begin{gathered}
f(\delta, \theta)=\delta^{2} f_{2}(1-\delta)+O\left(\delta^{4}\right), \\
m(\delta, \theta)=\delta^{2} m_{2}(1-3 \delta)+O\left(\delta^{4}\right), \\
l(\delta, \theta)=\delta^{2} l_{2}(1-3 \delta)+O\left(\delta^{4}\right), \\
\omega(\delta, \theta)=\omega_{\mathrm{H}}(1+\delta)+O\left(\delta^{2}\right), \\
H_{1}(\delta, \theta)=\delta\left(1-\frac{1}{2} \delta\right) H_{11}+O\left(\delta^{3}\right), \\
H_{2}(\delta, \theta)=H_{20}+O\left(\delta^{2}\right), \\
H_{3}(\delta, \theta)=H_{30}+O\left(\delta^{2}\right), \\
H_{4}(\delta, \theta)=H_{40}+O\left(\delta^{2}\right), \\
\bar{B}_{1}(\delta, \theta)=-\frac{\omega_{\mathrm{H}} \cos \theta}{x_{\mathrm{H}}}+O\left(\delta^{2}\right), \\
\bar{B}_{2}(\delta, \theta)=\frac{\omega_{\mathrm{H}} \sin \theta}{x_{\mathrm{H}}}+O\left(\delta^{2}\right) .
\end{gathered}
$$

The expansion coefficients $f_{2}, m_{2}, l_{2}, H_{11}, H_{20}, H_{30}$, and $H_{40}$ are functions of the variable $\theta$. Among these coefficients the following relations hold,

$$
\begin{gathered}
0=\frac{\partial_{\theta} m_{2}}{m_{2}}-2 \frac{\partial_{\theta} f_{2}}{f_{2}} \\
H_{11}=\partial_{\theta} H_{20} .
\end{gathered}
$$

Further details of the expansion at the horizon are given in Appendix C.

\subsubsection{Horizon properties}

Let us now obtain the horizon properties of the EYM solutions from the expansion at the horizon of the metric and gauge field functions.

The first quantity of interest is the area of the horizon. The dimensionless area $A$ is given by

$$
A=2 \pi \int_{0}^{\pi} d \theta \sin \theta \frac{\sqrt{l_{2} m_{2}}}{f_{2}} x_{\mathrm{H}}^{2} .
$$


The area $A$ of the black hole horizon defines the area parameter $x_{\Delta}$ via

$$
A=4 \pi x_{\Delta}^{2} .
$$

The entropy $S$ of the black hole then corresponds to

$$
S=\frac{A}{4} \text {. }
$$

To obtain a measure for the deformation of the horizon we compare the dimensionless circumference of the horizon along the equator, $L_{e}$, with the dimensionless circumference of the horizon along the poles, $L_{p}$,

$$
L_{e}=\left.\int_{0}^{2 \pi} d \varphi \sqrt{\frac{l}{f}} x \sin \theta\right|_{x=x_{\mathrm{H}}, \theta=\pi / 2}, \quad L_{p}=\left.2 \int_{0}^{\pi} d \theta \sqrt{\frac{m}{f}} x\right|_{x=x_{\mathrm{H}}, \varphi=\text { const. }},
$$

and consider, in particular, their ratio $L_{e} / L_{p}$.

The surface gravity of the black hole solutions is obtained from [19]

$$
\kappa_{\mathrm{sg}}^{2}=-1 / 4\left(D_{\mu} \chi_{\nu}\right)\left(D^{\mu} \chi^{\nu}\right),
$$

with Killing vector $\chi=\xi-\left(\omega_{\mathrm{H}} / x_{\mathrm{H}}\right) \eta$. Inserting the expansion in $\delta=\left(x / x_{\mathrm{H}}-1\right)$ at the horizon, Eqs. (66)-(69), yields for the dimensionless surface gravity

$$
\kappa_{\mathrm{sg}}=\frac{f_{2}(\theta)}{x_{\mathrm{H}} \sqrt{m_{2}(\theta)}} .
$$

As seen from Eq. (76), $\kappa_{\mathrm{sg}}$ is indeed constant on the horizon, as required by the zeroth law of black hole mechanics. The dimensionless temperature $T$ of the black hole is proportional to the surface gravity,

$$
T=\frac{\kappa_{\mathrm{sg}}}{2 \pi} .
$$

Let us now consider the Yang-Mills horizon charges. By evaluating the integrals, Eqs. (48) and (50), at the horizon, we obtain the horizon electric charge $Q_{\Delta}$ and the horizon magnetic charge $P_{\Delta}$ [17], respectively,

$$
\begin{gathered}
Q_{\Delta}=\frac{e}{4 \pi} \oint \sqrt{\sum_{i}\left({ }^{*} F_{\theta \varphi}^{i}\right)^{2}} d \theta d \varphi, \\
P_{\Delta}=\frac{e}{4 \pi} \oint \sqrt{\sum_{i}\left(F_{\theta \varphi}^{i}\right)^{2}} d \theta d \varphi,
\end{gathered}
$$

where $Q_{\Delta}$ and $P_{\Delta}$ again represent the dimensionless quantities. 


\subsection{Local charges}

To define the local mass let us first consider the general definition of the total mass (see e. g. [19])

$$
\mathcal{M}=2 \int_{\Sigma}\left(T_{\mu \nu}-\frac{1}{2} T g_{\mu \nu}\right) n^{\mu} \xi^{\nu} d V-\frac{1}{8 \pi G} \int_{\mathrm{H}} \frac{1}{2} \varepsilon_{\mu \nu \rho \sigma} \nabla^{\rho} \xi^{\sigma} d x^{\mu} d x^{\nu},
$$

where $\Sigma$ denotes an asymptotically flat hypersurface bounded by the horizon $\mathrm{H}, d V$ is the natural volume element on $\Sigma, n^{\mu}=(1,0,0,-\omega / r) / \sqrt{f}$ is normal to $\Sigma$ with $n_{\mu} n^{\mu}=-1$, and $\xi^{\nu}$ denotes the time like Killing vector field.

Note, that $T=0$ for the action, Eq. (11). Straightforward calculation yields

$$
T_{\mu \nu} n^{\mu} \xi^{\nu} d V=-T_{0}^{0} \sqrt{-g} d r d \theta d \varphi=-\frac{1}{8 \pi G} R_{0}^{0} \sqrt{-g} d r d \theta d \varphi,
$$

where in the last step the Einstein equations have been used.

Integration over $\Sigma$ yields

$-\frac{1}{8 \pi G} \int_{\Sigma} R_{0}^{0} \sqrt{-g} d r d \theta d \varphi=\left.\frac{2 \pi}{4 \pi G} \int_{0}^{\frac{\pi}{2}}\left[\frac{\sqrt{l}}{f} r^{2} \sin \theta\left(\frac{\partial f}{\partial r}-\frac{l}{f} \sin ^{2} \theta \omega\left(\frac{\partial \omega}{\partial r}-\frac{\omega}{r}\right)\right)\right]\right|_{r_{\mathrm{H}}} ^{\infty} d \theta$.

The calculation of the boundary term in Eq. (87) yields the same expression, but evaluated at $r=r_{\mathrm{H}}$.

Consequently ,

$$
\begin{aligned}
\mathcal{M} & =\left.\lim _{r \rightarrow \infty} \frac{2 \pi}{4 \pi G} \int_{0}^{\frac{\pi}{2}}\left[\frac{\sqrt{l}}{f} r^{2} \sin \theta\left(\frac{\partial f}{\partial r}-\frac{l}{f} \sin ^{2} \theta \omega\left(\frac{\partial \omega}{\partial r}-\frac{\omega}{r}\right)\right)\right]\right|_{r} d \theta \\
& =\left.\lim _{x \rightarrow \infty} \frac{\sqrt{4 \pi G}}{e} \frac{1}{G} \frac{1}{2} \int_{0}^{\frac{\pi}{2}}\left[\frac{\sqrt{l}}{f} x^{2} \sin \theta\left(\frac{\partial f}{\partial x}-\frac{l}{f} \sin ^{2} \theta \omega\left(\frac{\partial \omega}{\partial x}-\frac{\omega}{x}\right)\right)\right]\right|_{x} d \theta \\
& =\lim _{x \rightarrow \infty} \frac{\sqrt{4 \pi G}}{e} \frac{1}{G} M(x),
\end{aligned}
$$

where we changed to the dimensionless coordinate $x$, Eq. (31), and defined the dimensionless local mass $M(x)$,

$$
M(x)=\frac{1}{2} \int_{0}^{\frac{\pi}{2}} \frac{\sqrt{l}}{f} x^{2} \sin \theta\left(\frac{\partial f}{\partial x}-\frac{l}{f} \sin ^{2} \theta \omega\left(\frac{\partial \omega}{\partial x}-\frac{\omega}{x}\right)\right) d \theta .
$$

In a similar way we obtain the dimensionless local angular momentum $J(x)$,

$$
J(x)=\frac{1}{4} x^{2} \int_{0}^{\frac{\pi}{2}} \sin ^{3} \theta \frac{l^{3 / 2}}{f^{2}}\left(x \frac{\partial \omega}{\partial x}-\omega\right) d \theta .
$$


from the expression for the total angular momentum,

$$
\mathcal{J}=-\left[\frac{1}{8 \pi G} \int_{\Sigma} R_{\mu \nu} n^{\mu} \eta^{\nu} d V-\frac{1}{16 \pi G} \int_{\mathrm{H}} \frac{1}{2} \varepsilon_{\mu \nu \rho \sigma} \nabla^{\rho} \eta^{\sigma} d x^{\mu} d x^{\nu}\right]
$$

Defining the horizon mass of the black hole $M_{\Delta}=M\left(x_{\mathrm{H}}\right)$ and its horizon angular momentum $J_{\Delta}=J\left(x_{\mathrm{H}}\right)$, we obtain the relation

$$
M_{\Delta}=2 T S-2 \frac{\omega_{\mathrm{H}}}{x_{\mathrm{H}}} J_{\Delta} .
$$

We further define the dimensionless local non-Abelian electric and magnetic charges, $Q(x)$, and $P(x)$,

$$
Q(x)=\frac{e}{4 \pi} \oint \sqrt{\sum_{i}\left({ }^{*} F_{\theta \varphi}^{i}\right)^{2}} d \theta d \varphi
$$

and

$$
P(x)=\frac{e}{4 \pi} \oint \sqrt{\sum_{i}\left(F_{\theta \varphi}^{i}\right)^{2}} d \theta d \varphi,
$$

respectively, where the integrals are evaluated on surfaces with fixed radial coordinate $x$. Explicitly,

$$
\begin{aligned}
& Q(x)= \int_{0}^{\frac{\pi}{2}} \sin \theta \frac{\sqrt{l}}{f}\left\{\left[-x \sin ^{2} \theta \frac{\partial \omega}{\partial x} H_{3}+\sin \theta \cos \theta \omega\left(1-H_{4}\right)-\right.\right. \\
& x \sin \theta \cos \theta \frac{\partial \omega}{\partial x}\left(1-H_{4}\right)+\sin ^{2} \theta \omega H_{3}-x H_{1}\left(\cos \theta \bar{B}_{1}-\sin \theta \bar{B}_{2}\right)- \\
&\left.\omega H_{1}+x^{2}\left(\sin \theta \frac{\partial \bar{B}_{1}}{\partial x}+\cos \theta \frac{\partial \bar{B}_{2}}{\partial x}\right)\right]^{2}+\left[-x \sin \theta \cos \theta \frac{\partial \omega}{\partial x} H_{3}-\right. \\
& \sin ^{2} \theta \omega\left(1-H_{4}\right)+x \sin ^{2} \theta \frac{\partial \omega}{\partial x}\left(1-H_{4}\right)+\sin \theta \cos \theta \omega H_{3}+ \\
&\left.\left.x H_{1}\left(\sin \theta \bar{B}_{1}+\cos \theta \bar{B}_{2}\right)+x^{2}\left(\cos \theta \frac{\partial \bar{B}_{1}}{\partial x}-\sin \theta \frac{\partial \bar{B}_{2}}{\partial x}\right)\right]^{2}\right\}^{1 / 2} d \theta \\
& P(x)= \int_{0}^{\frac{\pi}{2}}\left\{\left[-\sin ^{2} \theta+\sin \theta \cos \theta H_{3}\left(1+H_{2}\right)+\cos { }^{2} \theta\left(H_{2}-H_{4}\right)+\right.\right. \\
&\left.\sin ^{2} \theta \frac{\partial H_{3}}{\partial \theta}-\sin \theta \cos \theta \frac{\partial H_{4}}{\partial \theta}+\sin ^{2} \theta H_{2} H_{4}\right]^{2}+[-\sin \theta \cos \theta- \\
& \sin ^{2} \theta H_{3}\left(1+H_{2}\right)+H_{3}-\sin \theta \cos \theta\left(H_{2}-H_{4}\right)+\sin \theta \cos \theta \frac{\partial H_{3}}{\partial \theta}+ \\
&\left.\sin ^{2} \theta \frac{\partial H_{4}}{\partial \theta}+\sin \theta \cos \theta H_{2} H_{4}\right]^{2} d \theta \\
& 1 / 2
\end{aligned}
$$




\section{Embedded Kerr-Newman Black Holes}

Here we briefly recall Kerr-Newman black holes, embedded in SU(2) EYM theory [22]. For better comparison with the non-Abelian rotating black holes, we first consider the coordinate transformation from Boyer-Lindquist coordinates to isotropic coordinates. Then we perform an SU(2) gauge transformation to the gauge employed for the nonAbelian black holes.

\subsection{Boyer-Lindquist coordinates}

In Boyer-Lindquist coordinates the metric of Kerr-Newman black holes is given by

$$
d s^{2}=-\frac{\Delta}{\rho^{2}}\left(d t+a \sin ^{2} \theta d \varphi\right)^{2}+\frac{\sin ^{2} \theta}{\rho^{2}}\left(a d t+\rho_{0}^{2} d \varphi\right)^{2}+\frac{\rho^{2}}{\Delta} d \tilde{x}^{2}+\rho^{2} d \theta^{2},
$$

where

$$
\rho^{2}=\tilde{x}^{2}+a^{2} \cos ^{2} \theta, \quad \rho_{0}^{2}=\tilde{x}^{2}+a^{2}, \quad \Delta=\tilde{x}^{2}-2 M \tilde{x}+a^{2}+C^{2},
$$

and $M$ denotes the black hole mass, $a$ the angular momentum per unit mass, $a=J / M$, and $C$ the "total charge" (see Eq. (98)).

The gauge field of embedded Kerr-Newman solutions is given by [22]

$$
A_{\mu}^{a} d x^{\mu}=\frac{Q^{a} \tilde{x}}{\rho^{2}}\left(d t+a \sin ^{2} \theta d \varphi\right)+\frac{P^{a} \cos \theta}{\rho^{2}}\left(a d t+\rho_{0}^{2} d \varphi\right),
$$

where $Q^{a}$ and $P^{a}$ are constant vectors in the Lie algebra, considered the Yang-Mills analogue of the electric and magnetic charge, respectively [22, 23]. They define the "total charge" $C$,

$$
C^{2}=Q^{a} Q^{a}+P^{a} P^{a}
$$

and $Q^{a}$ is proportional to $P^{a}$ 22.

The condition $\Delta\left(\tilde{x}_{\mathrm{H}}\right)=0$ yields the regular event horizon of the Kerr-Newman solutions,

$$
\tilde{x}_{\mathrm{H}}=M+\sqrt{M^{2}-\left(a^{2}+C^{2}\right)},
$$

extremal Kerr-Newman solutions satisfy $\tilde{x}_{\mathrm{H}}=M=\sqrt{a^{2}+C^{2}}$.

\subsection{Isotropic coordinates}

Let us now consider the Kerr-Newman solution in isotropic coordinates. For KerrNewman solutions the isotropic radial coordinate $x$ is related to the Boyer-Lindquist radial coordinate $\tilde{x}$ by

$$
x(\tilde{x})=\frac{1}{2}\left[\sqrt{\tilde{x}^{2}-2 M \tilde{x}+\left(a^{2}+C^{2}\right)}+(\tilde{x}-M)\right],
$$


and the metric functions $f(x, \theta), m(x, \theta), l(x, \theta)$ and $\omega(x, \theta)$ (see Eq. (8)) are given by

$$
\begin{gathered}
f(x, \theta)=\frac{\rho^{2} \Delta}{\rho_{0}^{4}-a^{2} \sin ^{2} \theta \Delta}, \\
\frac{m(x, \theta)}{x}=\frac{\tilde{x}^{2}+a^{2} \cos ^{2} \theta}{x^{2}}, \\
\frac{x^{2} l(x, \theta)}{f(x, \theta)}=\rho_{0}^{2}+a^{2} \sin ^{2} \theta \frac{2 M \tilde{x}-C^{2}}{\rho^{2}},
\end{gathered}
$$

and

$$
\frac{\omega(x, \theta)}{x}=\frac{a\left(2 M \tilde{x}-C^{2}\right)}{\rho_{0}^{4}-a^{2} \sin ^{2} \theta \Delta},
$$

where

$$
\tilde{x}(x)=x+M+\frac{M^{2}-\left(a^{2}+C^{2}\right)}{4 x} .
$$

In isotropic coordinates the event horizon resides at

$$
x_{\mathrm{H}}=1 / 2 \sqrt{M^{2}-\left(a^{2}+C^{2}\right)},
$$

thus extremal Kerr-Newman solutions satisfy $x_{\mathrm{H}}=0$. At the event horizon, the metric function $f$ is identically zero, the metric function ratios $(m / f)$ and $(l / f)$ are finite, and the metric function $\omega$ is constant,

$$
\omega\left(x_{\mathrm{H}}\right)=\frac{a x_{\mathrm{H}}}{4 M x_{\mathrm{H}}+2 M^{2}-C^{2}}=\omega_{\mathrm{H}} .
$$

Consequently the Kerr-Newman solutions satisfy

$$
\frac{\omega_{\mathrm{H}}}{x_{\mathrm{H}}}=\frac{\sqrt{M^{2}-4 x_{\mathrm{H}}^{2}-C^{2}}}{2 M\left(M+2 x_{\mathrm{H}}\right)-C^{2}}=\frac{a}{\left(M+2 x_{\mathrm{H}}\right)^{2}+a^{2}} .
$$

\subsection{Gauge transformation}

Let us now consider a Kerr-Newman solution with Lie algebra vectors $Q^{a}$ and $P^{a}$ pointing in the $z$-direction,

$$
A_{\mu} d x^{\mu}=\frac{Q \tilde{x}}{\rho^{2}}\left(d t+a \sin ^{2} \theta d \varphi\right) \frac{\tau_{z}}{2}+\frac{P \cos \theta}{\rho^{2}}\left(a d t+\rho_{0}^{2} d \varphi\right) \frac{\tau_{z}}{2} .
$$

To obtain the solution in the gauge employed for the non-Abelian ansatz (15)-(17), we perform a gauge transformation, where

$$
U(\theta, \varphi)=e^{-i \frac{\varphi}{2} \tau_{z}} e^{-i \frac{\theta}{2} \tau_{y}} .
$$


The gauge transformed vector field reads

$$
A^{\prime}{ }_{\mu} d x^{\mu}=\left(\frac{Q \tilde{x}}{\rho^{2}}+\frac{P \cos \theta}{\rho^{2}} a\right) \frac{\tau_{r}}{2} d t+A_{\varphi} d \varphi+\frac{\tau_{\varphi}}{2} d \theta
$$

with

$$
A_{\varphi}=\left(\frac{Q \tilde{x}}{\rho^{2}} a \sin ^{2} \theta+\frac{P \cos \theta}{\rho^{2}} \rho_{0}^{2}+\cos \theta\right) \frac{\tau_{r}}{2}-\sin \theta \frac{\tau_{\theta}}{2} .
$$

By comparing this embedded Abelian expression for the gauge fields with the nonAbelian ansatz, Eqs. (15)-(17), we obtain the embedded Abelian gauge field functions $H_{i}$ and $\bar{B}_{i}$,

$$
\begin{gathered}
H_{1}=H_{2}=H_{4}=0, \quad-\sin \theta H_{3}=\frac{Q \tilde{x}}{\rho^{2}} a \sin ^{2} \theta+\frac{P \cos \theta}{\rho^{2}} \rho_{0}^{2}+\cos \theta, \\
\bar{B}_{1}=\sin \theta \frac{\omega}{x} H_{3}+\frac{Q \tilde{x}+P \cos \theta a}{\rho^{2}}, \quad \bar{B}_{2}=\sin \theta \frac{\omega}{x} .
\end{gathered}
$$

The asymptotic expansion for the Abelian gauge field and metric functions is given in Appendix B, the expansion at the horizon in Appendix C.

\section{$5 \quad$ Numerical Results}

The rotating hairy black hole solutions of SU(2) EYM theory emerge from the static hairy black hole solutions, when a small value of the angular velocity of the horizon is imposed via the boundary conditions. We therefore briefly recall the properties of the static hairy black hole solutions of SU(2) EYM theory [4].

The static hairy black hole solutions of SU(2) EYM theory carry non-trivial magnetic gauge fields outside their regular event horizon, but carry no non-Abelian magnetic charge. Since their electric fields vanish identically, their only global charge is their mass. These black holes can be characterized by their mass and by two integers, the azimuthal winding number $n$ of their gauge fields [24] and the node number $k$ of their magnetic gauge field functions [4, 5, 25]. For a given winding number $n$, the solutions form sequences labelled by the node number $k$. With increasing $k$, the non-Abelian black hole solutions approach a limiting solution, corresponding to an embedded Abelian solution with magnetic charge $n$ [26].

In contrast to the static hairy black hole solutions, their rotating generalizations carry electric gauge fields. Notably, the rotating hairy black hole solutions possess a non-Abelian electric charge [12, 16, whereas their static counterparts are neutral. Thus their global charges are their mass, their angular momentum and their non-Abelian electric charge. Their non-Abelian magnetic charge is identically zero. Like their static 
counterparts, the rotating hairy black hole solutions form sequences depending on the winding number $n$ and the node number $k$ of the gauge fields.

Here we consider the sequence of rotating hairy black hole solutions with winding number $n=1$ and node number $k$. In the static limit these black hole solutions approach spherically symmetric SU(2) EYM black hole solutions [4]. Rotating black hole solutions with higher winding number $n$ approach in the static limit SU(2) EYM black hole solutions, which possess only axial symmetry [5]. Such black hole solutions will be considered elsewhere.

For a given node number $k$, the rotating hairy black hole solutions depend on the isotropic horizon radius $x_{\mathrm{H}}$ and on the value of the metric function $\omega$ at the horizon, $\omega_{\mathrm{H}}$, via the boundary conditions. The ratio $\omega_{\mathrm{H}} / x_{\mathrm{H}}$ represents the rotational velocity of the horizon.

When the black hole solutions are constructed, their global charges are obtained from the asymptotic fall-off of the metric and gauge field functions of the solutions. Likewise, their horizon properties are obtained from the expansion at the horizon.

To construct the rotating hairy black hole solutions, we solve the set of ten coupled non-linear elliptic partial differential equations numerically [27], subject to the above boundary conditions. We employ compactified dimensionless coordinates, mapping spatial infinity to the finite value $\bar{x}=1$, where

$$
\bar{x}=1-\frac{x_{\mathrm{H}}}{x} .
$$

We furthermore introduce the functions $\bar{f}(\bar{x}, \theta), \bar{l}(\bar{x}, \theta), \bar{m}(\bar{x}, \theta)$, and $g(\bar{x}, \theta)$ [18, 5, , 7 ,

$$
\bar{f}(\bar{x}, \theta)=\frac{f(\bar{x}, \theta)}{\bar{x}^{2}}, \quad \bar{l}(\bar{x}, \theta)=\frac{l(\bar{x}, \theta)}{\bar{x}^{2}}, \quad \bar{m}(\bar{x}, \theta)=\frac{m(\bar{x}, \theta)}{\bar{x}^{2}}, \quad g(\bar{x}, \theta)=\frac{m(\bar{x}, \theta)}{l(\bar{x}, \theta)} .
$$

The numerical calculations, based on the Newton-Raphson method, are performed with help of the program FIDISOL 27. The equations are discretized on a non-equidistant grid in $\bar{x}$ and $\theta$. Typical grids used have sizes $100 \times 20$, covering the integration region $0 \leq \bar{x} \leq 1$ and $0 \leq \theta \leq \pi / 2$. (See [18, 5, 4] and [27] for further details on the numerical procedure.)

\subsection{Global charges}

To construct a rotating black hole solution with one node and horizon radius $x_{\mathrm{H}}$, we start from the static spherically symmetric SU(2) EYM black hole solution with one node and the same horizon radius, which represents the limiting solution in the static limit $\omega_{\mathrm{H}} \rightarrow 0$. By imposing a small but finite value of $\omega_{\mathrm{H}}$ via the boundary conditions, a rotating black hole solution is obtained, which possesses non-trivial functions $\omega, H_{1}$, $H_{3}, \bar{B}_{1}, \bar{B}_{2}$. The rotating black hole solution is only axially symmetric. It possesses a non-vanishing electric gauge field, giving rise to a non-Abelian electric charge [12, 16]. 
When increasing $\omega_{\mathrm{H}}$ from zero, while keeping $x_{\mathrm{H}}$ fixed, a branch of black hole solutions forms, the lower branch. The lower branch extends up to a maximal value $\omega_{\mathrm{H}}^{\max }$, where a second branch, the upper branch, bends backwards towards $\omega_{\mathrm{H}}=0$. Along both branches the mass $M$, the angular momentum $J$, and the non-Abelian electric charge $Q$ continuously increase [16]. This is seen in Figs. 1a-c, where the mass $M$, the angular momentum per unit mass $a=J / M$ and the non-Abelian electric charge $Q$ are shown as functions of the parameter $\omega_{\mathrm{H}}$ for three values of the isotropic horizon radius, $x_{\mathrm{H}}=0.1,0.5$ and 1 .

Whereas both mass $M$ and angular momentum per unit mass $a$ of the non-Abelian solutions increase strongly along the upper branch, diverging with $\omega_{\mathrm{H}}^{-1}$ in the limit $\omega_{\mathrm{H}} \rightarrow 0$, the non-Abelian electric charge $Q$ remains small. It approaches apparently a finite limiting value, $Q_{\lim } \approx 0.124$ (as discussed in section 5.3), independent of the isotropic horizon radius $x_{\mathrm{H}}$.

For comparison, we exhibit in Figs. 1a-b also the mass and the angular momentum of embedded Abelian solutions with the same horizon radii: those of the Kerr solutions and those of the Kerr-Newman solutions with $Q=0$ and $P=-1$. (Mass and angular momentum of embedded Kerr-Newman solutions, possessing the same charge $Q$ as the non-Abelian solutions but with $P=0$ are graphically indistinguishable from the mass and angular momentum of the Kerr solutions, shown. Likewise, mass and angular momentum of embedded Kerr-Newman solutions, possessing the same charge $Q$ as the non-Abelian solutions and $P=-1$ are graphically indistinguishable from the mass and angular momentum of the Kerr-Newman solutions, shown.)

Interestingly, both mass and angular momentum of the non-Abelian solutions, which carry a small electric charge $Q$ and no magnetic charge, are close to mass and angular momentum of the embedded Kerr-Newman solutions with magnetic charge $P=-1$. This is seen in particular for solutions with small horizon radii $x_{\mathrm{H}}$, where large deviations of these global properties from those of the corresponding Kerr solutions arise. For non-Abelian black hole solutions with large horizon radii, these global charges are also close to those of the Kerr solutions. Here a magnetic charge of magnitude $|P|=1$ is less important for the global properties of the solutions. Thus also Kerr and Kerr-Newman solutions differ less for large horizon radii.

As a point in case, let us inspect the maximal value of $\omega_{\mathrm{H}}$ reached, $\omega_{\mathrm{H}}^{\max }$. The maximal value $\omega_{\mathrm{H}}^{\max }$ of the non-Abelian black hole solutions depends on the horizon radius $x_{\mathrm{H}}$, and increases monotonically with $x_{\mathrm{H}}$. For the solutions shown in Figs. 1a-c, $\omega_{\mathrm{H}}^{\max }\left(x_{\mathrm{H}}=0.1\right) \approx 0.0288, \omega_{\mathrm{H}}^{\max }\left(x_{\mathrm{H}}=0.5\right) \approx 0.0649$, and $\omega_{\mathrm{H}}^{\max }\left(x_{\mathrm{H}}=1.0\right) \approx 0.0719$. For Kerr-Newman solutions with $Q=0$ and $P=-1$ very similar values for $\omega_{\mathrm{H}}^{\max }$ are obtained, $\omega_{\mathrm{H}}^{\max }\left(x_{\mathrm{H}}=0.1\right) \approx 0.0278, \omega_{\mathrm{H}}^{\max }\left(x_{\mathrm{H}}=0.5\right) \approx 0.0646$, and $\omega_{\mathrm{H}}^{\max }\left(x_{\mathrm{H}}=1.0\right) \approx$ 
0.0718. In contrast, for the Kerr solutions the maximal value of $\omega_{\mathrm{H}}$,

$$
\omega_{\mathrm{H}}^{\max , \mathrm{K}}=\frac{1}{2(\sqrt{5}+1)} \sqrt{\frac{\sqrt{5}-1}{\sqrt{5}+3}} \approx 0.07507,
$$

is independent of the horizon radius $x_{\mathrm{H}}$. We conjecture that, in the limit $x_{\mathrm{H}} \rightarrow \infty$, the

maximal value $\omega_{\mathrm{H}}^{\max }$ of the non-Abelian solutions tends to the maximal value $\omega_{\mathrm{H}}^{\max , \mathrm{K}}$ of the Kerr solutions.

So far we have limited the discussion to non-Abelian solutions with one node. When we consider black hole solutions with higher node numbers, we observe similar features. The rotating hairy black hole solution with $k$ nodes emerges from the corresponding static black hole solution, when a finite value of $\omega_{\mathrm{H}}$ is imposed via the boundary conditions. When $\omega_{\mathrm{H}}$ increases, the lower branch of black hole solutions forms. It extends up to a maximal value $\omega_{\mathrm{H}}^{\max }$, where the upper branch forms and bends backwards towards $\omega_{\mathrm{H}}=0$. Along both branches mass, angular momentum, and non-Abelian electric charge continuously increase.

For node number $k=3$, the mass and angular momentum of the black hole solutions differ only little from the mass and angular momentum of the $k=1$ black hole solutions. In general, the values are still closer to those of the Kerr-Newman solutions with $Q=0$ and $P=-1$. Also, the values of $\omega_{\mathrm{H}}^{\max }$ are very close to those of the Kerr-Newman solutions with $Q=0$ and $P=-1$. Along the lower branch, the non-Abelian electric charge of the $k=3$ solutions is much smaller than the electric charge of the $k=1$ solutions, typically it is on the order of $10^{-4}$. Along the upper branch the non-Abelian electric charge increases relatively strongly. However, because of numerical inaccuracies encountered along the upper branch for the higher node solutions, we cannot infer from the numerical calculations, that the charge tends to a limiting value $Q_{\text {lim. }}$.

\section{$5.2 \quad$ Energy density}

Let us now discuss the rotating black hole solutions in more detail and begin with the energy density of the black hole solutions, $\varepsilon=-T_{0}^{0}$.

Due to the rotation, the energy density of the matter fields is angle-dependent, and, in particular, not constant at the horizon. The maximum of the energy density resides on the $\rho$-axis at the horizon, as seen in the following two representative examples.

In Figs. 2a-d we exhibit the energy density of the matter fields of the black hole solution with horizon radius $x_{\mathrm{H}}=1$ and $\omega_{\mathrm{H}}=0.04$ on the lower branch. Fig. 2a shows a 3-dimensional plot of the energy density as a function of the coordinates $\rho=x \sin \theta$ and $z=x \cos \theta$ together with a contour plot, and Figs. 2b-d show surfaces of constant energy density. The surfaces of constant energy density appear ellipsoidal, being flatter at the poles than in the equatorial plane. For the largest values of the energy density, as shown in Fig. 2d, the horizon is seen in the pole region. 
Analogously, we exhibit in Figs. 3a-d the energy density of the matter fields of the black hole solution with horizon radius $x_{\mathrm{H}}=1$ and $\omega_{\mathrm{H}}=0.04$ on the upper branch. The energy density is much stronger deformed now, showing a large peak on the $\rho$-axis. Consequently, the surfaces of constant energy density appear torus-shaped, with the horizon seen in the center of the torus. Further away form the black hole horizon, however, the surfaces of constant energy density appear again ellipsoidal,

We do not observe a strong dependence of the energy density on the node number $k$. On the lower branch the $k=3$ densities are similar to the $k=1$ densities. On the upper branch, the numerical determination of the $k=3$ densities becomes, however, unreliable.

\subsection{Gauge field and metric functions}

We now turn to the metric and gauge field functions of the non-Abelian black hole solutions. In Figs. 4a-j we show the functions for the solutions with one node on the upper and lower branch for horizon radius $x_{\mathrm{H}}=1$ and $\omega_{\mathrm{H}}=0.04$. Also shown are the functions of the three node solution on the lower branch for the same set of parameters. We first discuss the magnetic gauge field functions, shown in Figs. 4a$4 \mathrm{~d}$, then the electric gauge field functions, shown in Figs. 4e-4f, and finally the metric functions, shown in Figs. $4 \mathrm{~g}-4 \mathrm{j}$. Since the global mass and angular momentum of the non-Abelian solutions are rather close to those of the Kerr-Newman solutions with $Q=0$ and $P=-1$, we compare with the corresponding functions of these KerrNewman solutions.

The magnetic gauge field function $H_{1}$, shown in Fig. 4a, is always very small. For the $k=1$ solutions, its magnitude is two orders of magnitude smaller on the lower branch than on the upper branch. For the $k=3$ lower branch solution it is still smaller by two orders of magnitude than for the lower branch $k=1$ solution. $H_{1}$ shows a distinct angle dependence, which is similar for the three black holes exhibited. The Kerr-Newman solutions have vanishing $H_{1}$. This is consistent with the observed smallness of $H_{1}$ for the non-Abelian solutions.

The magnetic gauge field function $H_{2}$ is almost spherically symmetric for the $k=1$ solutions on both branches as well as for the $k=3$ solution on the lower branch. It is exhibited in Fig. 4b. Whereas $H_{2}$ decreases monotonically for the $k=1$ solutions from a finite positive value at the horizon to its boundary value of -1 at infinity, $H_{2}$ oscillates around zero (possessing three nodes) for the $k=3$ solution in an extended interior region, before it decreases to its boundary value -1 for $x \rightarrow \infty$. Thus $H_{2}$ precisely keeps its features of the static non-Abelian solutions, where with increasing node number $k$, it becomes close to zero in an increasing interior region. In particular, in the limit $k \rightarrow \infty$, a limiting solution with $H_{2}=0$ is reached [26].

The magnetic gauge field function $H_{3}$, shown in Fig. 4c, is very similar for the $k=1$ 
and $k=3$ solutions on the lower branch, and about an order of magnitude smaller on the lower branch than on the upper branch. Comparison of the function $\mathrm{H}_{3}$ of the nonAbelian solutions with the function $\mathrm{H}_{3}$, Eq. (113), of the corresponding Kerr-Newman solutions with $Q=0$ and $P=-1$ reveals very good agreement. Thus $H_{3}$, like $H_{1}$, corresponds approximately to the Abelian function.

The magnetic gauge field function $H_{4}$, shown in Fig. $4 \mathrm{~d}$, is rather similar to the function $\mathrm{H}_{2}$ on the lower branch, both for the $k=1$ and the $k=3$ solutions. Its angle dependence on the lower branch is only small. On the upper branch, however, a distinct angle dependence appears. $H_{4}$, like $H_{2}$, retains the non-Abelian character of the solutions.

Turning to the electric gauge field functions $\bar{B}_{1}$ and $\bar{B}_{2}$ we observe, that the angle dependence is largely determined by the boundary conditions at the horizon. To eliminate this trivial angle dependence, we consider the new functions $\hat{B}_{1}$ and $\hat{B}_{2}$, defined via

$$
\hat{B}_{1}=-\cos \theta \bar{B}_{1}+\sin \theta \bar{B}_{2}, \quad \hat{B}_{2}=\sin \theta \bar{B}_{1}+\cos \theta \bar{B}_{2} .
$$

As seen in Fig. 4e, the function $\hat{B}_{1}$ then shows only a relatively small deviation from spherical symmetry for all three black hole solutions considered. The function $\hat{B}_{2}$ is considerably smaller in amplitude than the function $\hat{B}_{1}$. Its angle dependence is similar for the $k=1$ and $k=3$ solutions on the lower branch, but different on the upper branch.

Let us again compare with the electric gauge field functions of the corresponding Kerr-Newman solutions, Eq. (114), with $Q=0$ and $P=-1$. We again find good agreement between these Abelian and the non-Abelian functions. Thus $\hat{B}_{1}$ and $\hat{B}_{2}$ also correspond in good approximation to the Abelian functions.

Thus we conclude, that the non-Abelian gauge field functions, which are nonvanishing in the static limit, $H_{2}$ and $H_{4}$, retain their non-Abelian features. Whereas all gauge field functions which vanish in the static limit, $H_{1}, H_{3}, B_{1}$ and $B_{2}$, are very close to the corresponding functions of the Kerr-Newman solution with $Q=0$ and $P=-1$.

Let us now turn to the metric functions. In order to exhibit the behaviour of the metric functions $f, m$ and $l$ at the horizon more clearly we show the functions $\bar{f}(x, \theta)$, $\bar{l}(x, \theta)$, and $g(x, \theta)$, Eq. (116).

The function $\bar{f}$ is shown in Fig. $4 \mathrm{~g}$. It is almost spherically symmetric on the lower branch and deviates from spherical symmetry only slightly on the upper branch. On the upper branch the magnitude of $\bar{f}$ and its slope at the horizon are considerably smaller than on the lower branch. There is very little dependence of the metric function $\bar{f}$ on the node number. This is also true for the other metric functions.

In Fig. 4 he show the ratio $g=m / l$. Note, that for a spherically symmetric metric the function $g$ is identical to one, thus the deviation of this function from one indicates the deviation from spherical symmetry. We observe that on the lower branch $g$ is close to one for all $x$ except in a region near the horizon, where it deviates slightly from one. On the upper branch, in contrast, the deviation from one is large and $g$ approaches its 
asymptotic value only for larger values of $x$.

The metric function $\bar{l}$ is shown in Fig. 4i. For solutions with $k=1$ and $k=3$ nodes, as well as for solutions on the lower and upper branch, the functions $\bar{l}$ are almost identical. Also, their deviation from spherical symmetry is extremely small.

We exhibit the function $\omega$ in Fig. 4j. The shape of the function $\omega$ is similar for solutions on the lower and upper branch. However, on the upper branch the maximum is larger than on the lower branch. The angle dependence is small, except near the maximum of the function on the upper branch.

In general, the deviation from spherical symmetry is small on the lower branch. We also observe little dependence of the metric functions on the node number, although for solutions with smaller horizon radius the node number dependence of the metric functions increases slightly. All metric functions are rather close to the corresponding functions of the Kerr-Newman solutions with $Q=0$ and $P=-1$.

We conclude, that the functions of the non-Abelian solutions are rather close to those of the embedded Kerr-Newman solutions with $Q=0$ and $P=-1$, except for those gauge field functions, which do not vanish in the static limit. It is surprising, that already the one node solutions follows so closely these Abelian solutions, because their functions $H_{2}$ and $H_{4}$ are still very different from zero, the value assumed by the Abelian solutions, Eq. (113). With increasing node number, though, one expects the solutions to get close to the Abelian solution, since $H_{2}$ and $H_{4}$ are close to zero in an increasing interval.

Let us now address the limiting non-Abelian solutions, obtained in the limit $\omega_{\mathrm{H}} \rightarrow$ 0. Along the lower branch the limiting solution corresponds to a static spherically symmetric non-Abelian black hole solution. In contrast, along the upper branch mass and angular momentum of the solution diverges, thus the limiting solution is singular. Comparison with the Abelian solutions shows, that in the limit $\omega_{\mathrm{H}} \rightarrow 0$, electric and magnetic charge become negligible when $Q \ll 1$ and $|P|=1$. Consequently, the ratio $M / a$ tends to one for fixed $x_{\mathrm{H}}$.

We gain some understanding of the limiting solution by noting, that it can be obtained numerically, as a non-Abelian gauge field solution in an extremal background metric. Scaling the radial coordinate and angular momentum per unit mass by the mass, and taking the limit $M \rightarrow \infty$, the metric decouples from the gauge field, i. e. $G_{\mu \nu}=0$. Taking the limiting extremal Kerr solution as background metric, one can solve the gauge field equations numerically. The corresponding solution is then independent of the isotropic horizon radius $x_{\mathrm{H}}$. The limiting value of the electric charge obtained in this way is $Q_{\lim }=0.124$. 


\subsection{Local charges}

In Figs. 5a-d we present the local charges $M(x), J(x), Q(x)$ and $P(x)$, Eqs. (88)-(89) and Eqs. (93)-(94), for the black hole solutions with one node on the lower and upper branch, for horizon radius $x_{\mathrm{H}}=1$ and $\omega_{\mathrm{H}}=0.04$. We also show these charges for the black hole solution with three nodes on the lower branch for the same set of parameters.

In Fig. 5a the local mass $M(x)$ is shown. We observe, that the mass at infinity, representing the global mass, is only slightly larger than the mass at the horizon. For the solutions shown, we find on the lower branch the global masses $M_{k=1} \approx 2.357$ and $M_{k=3} \approx 2.371$, and on the upper branch we find $M_{k=1} \approx 10.25$. Thus the fields outside the horizon contribute little to the mass.

In Fig. 5b the local angular momentum $J(x)$ is shown. Again, the global angular momentum, read off at infinity, is only slightly larger than the angular momentum at the horizon. For the solutions shown, the global angular momentum on the lower branch is $J_{k=1} \approx 1.852$ and $J_{k=3} \approx 1.872$, and on the upper branch it is $J_{k=1} \approx 102.5$.

The local electric charge $Q(x)$ is shown in Fig. 5c. For solutions on both branches $Q(x)$ decreases significantly with increasing $x$, giving rise to a large difference between the horizon electric charge and the global electric charge. In general $Q(x)$ is smaller on the lower branch than on the upper branch, where it assumes values between $\approx 0.9$ at the horizon and 0.09 at infinity for the solutions shown. The decrease with $x$ indicates the presence of a charge density outside the horizon, cancelling part of the horizon charge.

We show the local magnetic charge $P(x)$ in Fig. 5d. In contrast to the local electric charge, the local magnetic charge is not monotonic but possesses a maximum of magnitude one, for the solutions on both branches. At infinity $P(x)$ vanishes, in accordance with the boundary conditions, corresponding to a vanishing global magnetic charge. With increasing node number, the local magnetic charge remains close to the value one in an increasing region, as in the static case [3]. This again indicates, that in the limit $k \rightarrow \infty$, the rotating non-Abelian black hole solutions tend to a limiting solution with magnetic charge $|P|=1$, like their static counterparts [26].

\subsection{Horizon properties}

We now turn to the horizon properties of the rotating non-Abelian black holes. In Figs. 6a-d we demonstrate the dependence of the horizon mass, the horizon angular momentum, and the horizon electric and magnetic charges on $\omega_{\mathrm{H}}$ for fixed isotropic horizon radius $x_{\mathrm{H}}$, for black hole solutions with one node. The corresponding horizon size, the deformation of the horizon and the surface gravity are shown in Figs. 7a-c.

The horizon mass $M_{\Delta}$ follows closely the global mass $M$, as seen in Fig. 6a, where the horizon mass is shown for black hole solutions with horizon radius $x_{\mathrm{H}}=0.1$ and

$x_{\mathrm{H}}=1$. Similarly, the horizon angular momentum $J_{\Delta}$ closely follows the global an- 
gular momentum $J$. The horizon angular momentum per unit mass, $a_{\Delta}=J_{\Delta} / M_{\Delta}$, is shown in Fig. 6b. Both horizon mass and horizon angular momentum are close to the corresponding Kerr-Newman values for solutions with $Q=0$ and $P=-1$.

The horizon electric charge $Q_{\Delta}$, shown in Fig. 6c, increases monotonically along both branches, like the global non-Abelian electric charge $Q$. It is, however, about an order of magnitude larger than the global non-Abelian electric charge $Q$. The horizon magnetic charge $P_{\Delta}$ is shown in Fig. 6d. Whereas the black holes carry no global non-Abelian magnetic charge, their horizon magnetic charge is on the order of one. Starting from finite values on the lower branch, which correspond to the horizon magnetic charges of the static solutions, $P_{\Delta}$ decreases monotonically along the lower branch. It then reaches a minimum along the upper branch and increases again. The location of the minimum strongly depends on $x_{\mathrm{H}}$.

The horizon size as quantified by the area parameter $x_{\Delta}$ is shown in Fig. $7 \mathrm{a}$ for black holes with isotropic horizon radii $x_{\mathrm{H}}=0.1$ and $x_{\mathrm{H}}=1$ as a function of $\omega_{\mathrm{H}}$. The horizon area grows monotonically along both branches, and diverges along the upper branch. Comparison with the corresponding Abelian black hole solutions again shows, that the non-Abelian horizon size follows closely the Kerr-Newman horizon size for $Q=0$ and $P=-1$, whereas only for large $x_{\mathrm{H}}$ it is also close to the Kerr horizon size.

The deformation of the horizon is revealed by measuring the circumference of the horizon along the equator, $L_{e}$, and the circumference of the horizon along the poles, $L_{p}$, Eqs. (81). The deformation of the horizon, quantified by $L_{e} / L_{p}$, Eq. (81), is shown in Fig. 7b. The ratio $L_{e} / L_{p}$ grows monotonically along both branches. As $\omega_{\mathrm{H}}$ tends to zero on the upper branch, the ratio tends to the value $L_{e} / L_{p} \approx 1.645$, corresponding to the value of the limiting extremal Kerr solution, for $M \rightarrow \infty$. On the lower branch the ratio $L_{e} / L_{p}$ assumes the value one in the limit $\omega_{\mathrm{H}} \rightarrow 0$, corresponding to the value of a static spherically symmetric hairy black hole. Also shown are the corresponding embedded Abelian solutions, approaching the same limiting values of the deformation.

The surface gravity $\kappa_{\mathrm{sg}}$ is exhibited in Fig. 7c, along with the surface gravity of the Kerr and the Kerr-Newman solutions for $Q=0$ and $P=-1$. The surface gravity of the non-Abelian black holes decreases monotonically along both branches, starting from the value of the corresponding static non-Abelian black hole solution on the lower branch in the limit $\omega_{\mathrm{H}} \rightarrow 0$. On the upper branch the surface gravity tends to zero in the limit $\omega_{\mathrm{H}} \rightarrow 0$, the value assumed by extremal black hole solutions. The surface gravity of Kerr-Newman and non-Abelian solutions agrees well for black hole solutions with large horizon radii, for black hole solutions with small horizon radii, however, a difference is observed on the lower branch. Here, in the limit $\omega_{\mathrm{H}} \rightarrow 0$, the rotating non-Abelian black hole solutions approach the corresponding static non-Abelian black hole, and the Kerr-Newman solutions approach the corresponding Reissner-Nordstrøm black hole with $Q=0$ and $P=-1$. For these static solutions the difference in surface gravity in known to decrease with increasing horizon size [3]. 


\subsection{Fixed $\omega_{H}$}

Having considered the rotating hairy black hole solutions for fixed horizon radius $x_{\mathrm{H}}$ as a function of $\omega_{\mathrm{H}}$, let us now keep $\omega_{\mathrm{H}}$ fixed and vary the horizon radius.

In Fig. 8 we show the mass $M$ of the non-Abelian black hole solutions as a function of the isotropic horizon radius $x_{\mathrm{H}}$ for $\omega_{\mathrm{H}}=0.01, \omega_{\mathrm{H}}=0.02$, and $\omega_{\mathrm{H}}=0.05$. For a given value of $\omega_{\mathrm{H}}$ there is a minimal value of the horizon radius $x_{\mathrm{H}}$. In particular, the limit $x_{\mathrm{H}} \rightarrow 0$ is only reached for $\omega_{\mathrm{H}} \rightarrow 0$. Thus we do not obtain globally regular rotating solutions in the limit $x_{\mathrm{H}} \rightarrow 0$ [12].

For comparison, Fig. 8 also presents the mass $M$ of the corresponding Kerr solutions and Kerr-Newman solutions with $Q=0$ and $P=-1$. For a fixed value of $\omega_{\mathrm{H}}$, the mass of the Kerr solutions forms two straight lines, extending from the origin. The non-Abelian solutions tend toward these lines for large values of the horizon radius. The mass of the Kerr-Newman solutions again is close to the mass of the non-Abelian solutions. In particular, we observe, that the minimal values of the horizon radius only differ slightly for the non-Abelian and Kerr-Newman solutions.

\section{$5.7 \quad$ Fixed $\omega_{\mathrm{H}} / x_{\mathrm{H}}$}

Let us finally consider variation of the parameters $x_{\mathrm{H}}$ and $\omega_{\mathrm{H}}$, while keeping their ratio $\omega_{\mathrm{H}} / x_{\mathrm{H}}$ fixed.

The global charges of the black hole solutions with one node are shown in Fig. 9 for fixed ratio $\omega_{\mathrm{H}} / x_{\mathrm{H}}=0.04$. The mass $M$ and the angular momentum per unit mass $a$ increase monotonically along both branches, reaching finite limiting values on the upper branch for $\omega_{\mathrm{H}} \rightarrow 0$. Except in a small region close to $\omega_{\mathrm{H}}=0$ on the lower branch the non-Abelian electric charge $Q$ also increases monotonically. The non-Abelian electric charge also approaches a finite limiting value on the upper branch, apparently close to the limiting value $Q_{\text {lim }} \approx 0.124$, observed previously.

To gain a better understanding of the limiting behaviour on the upper branch, we consider also the horizon properties for these black hole solutions. In Fig. 10 we show their horizon size $x_{\Delta}$, their deformation $L_{e} / L_{p}$ and their surface gravity $\kappa_{\mathrm{sg}}$. On the upper branch the horizon size, the deformation and the surface gravity of the nonAbelian black hole solutions are very close to those of the Kerr-Newman solution with $Q=0$ and $P=-1$. In particular, the horizon size and deformation remain finite on the upper branch in the limit $\omega_{\mathrm{H}} \rightarrow 0$, and the surface gravity tends to zero. This indicates, that an extremal black hole is approached in this limit. Since the limiting black hole solution retains its non-Abelian character in the gauge field functions, it should correspond to a rotating hairy extremal black hole.

On the lower branch in the limit $\omega_{\mathrm{H}} \rightarrow 0$, the corresponding Bartnik-McKinnon solution is approached by the non-Abelian solutions, whereas the Kerr-Newman solutions approach a Reissner-Nordstrøm solution. This limiting behaviour is suggested by 
a detailed inspection of the metric and gauge field functions. In Fig. 10 this limiting behaviour is reflected in the fact, that the horizon size of the non-Abelian solutions tends to zero, whereas the horizon size of the Kerr-Newman solutions tends to a finite value. Furthermore, the surface gravity of the non-Abelian solutions apparently diverges in the limit, in agreement with the static non-Abelian results [3], whereas the surface gravity of the Kerr-Newman solutions tends to zero, the value of an extremal Reissner-Nordstrøm solution.

\section{Conclusions}

We have given a detailed account of a new class of black hole solutions in SU(2) EYM theory, which represent the first examples of non-perturbative stationary non-Abelian black hole solutions [5]. These black hole solutions carry mass, angular momentum and a non-Abelian electric charge. Although they do not carry a non-Abelian magnetic charge, they still possess non-trivial magnetic gauge fields outside their regular event horizon. They therefore represent rotating hairy black hole solutions.

The global charges of the rotating hairy black hole solutions are not independent. For a given mass and angular momentum, as well as node number of the solution, a unique electric charge is obtained. Whereas mass and angular momentum are unbounded, we observe, that the electric charge remains very small.

The event horizon of the static axially symmetric black hole solutions resides at a surface of constant isotropic radial coordinate, $x=x_{\mathrm{H}}$. The boundary conditions at the horizon ensure regularity of the horizon. The horizon mass and horizon angular momentum are only slightly smaller than the global mass and the global angular momentum of the black hole solutions. The horizon electric charge is, however, significantly larger than the global electric charge, and the solutions possess horizon magnetic charge of order one, whereas their global magnetic charge vanishes.

The rotating hairy black hole solutions emerge from the static hairy black hole solutions in the limit of vanishing angular momentum. Since the static spherically symmetric black hole solutions form a sequence labelled by the node number $k$ of the gauge field function, we obtain the corresponding sequence of rotating black hole solutions, by starting from the static black hole solutions and imposing a small angular velocity of the horizon via the boundary conditions.

The rotating non-Abelian black hole solutions are rather close to the Kerr-Newman solutions with $Q=0$ and $P=-1$. In particular, the metric and gauge field functions of the non-Abelian solutions are very close to those of the Abelian solutions, except for those gauge field function, which do not vanish in the static limit. These retain the non-Abelian character of the solutions. However, with increasing node number, also these functions tend to their Abelian counterparts. 
The asymptotic expansion performed for the metric and gauge field functions, contains non-integer powers for the magnetic gauge field functions. In particular, the non-integer exponents depend on the non-Abelian electric charge. The expansion then imposes constraints on the possible values of the electric charge. Since in the static limit the non-Abelian electric charge vanishes, the well known power law decay of the static gauge field functions is recovered.

The expansions of the metric and gauge field functions at the horizon show, that the rotating hairy black hole solutions satisfy the zeroth law of black hole mechanics [19. Relations obtained recently within the isolated horizon framework [17, 21] concerning various horizon properties, such as the horizon mass and the horizon charges, will be considered elsewhere.

The hairy black hole solutions constructed here non-perturbatively, were first considered perturbatively [12]. To compare with these perturbative calculations, where linear rotational excitations of the static EYM black holes were studied, we consider the slowly rotating non-Abelian solutions in the limit $\omega_{\mathrm{H}} \rightarrow 0$. In the perturbative calculations $Q \propto J[12]$, and the ratio $Q / J$ depends only on the horizon radius. The non-perturbative calculations show good agreement with the non-perturbative results for the slowly rotating solutions with large values of the horizon radius.

Besides these non-Abelian stationary black hole solutions with finite angular momentum $J$ and finite electric charge $Q$, perturbative studies [15] have predicted two more types of stationary non-Abelian black hole solutions. These correspond to rotating black hole solutions which are uncharged $(J>0, Q=0)$, and non-static charged black hole solutions, which have vanishing angular momentum $(J=0, Q \neq 0)$. Both types satisfy a different set of boundary conditions at infinity.

This different set of boundary conditions at infinity should also be observed by rotating regular non-Abelian solutions [15]. The numerical construction of such nonperturbative regular solutions has been attempted recently [28], and arguments have been put forward, that such solutions should not exist. Our attempts to obtain numerically the non-perturbative counterparts of the predicted further types of black hole solutions have met with the same difficulties for the same reasons [29].

Besides the rotating black hole solutions considered here, there might be rapidly rotating branches of non-Abelian black holes solutions in EYM theory, not connected to the static solutions. There should also be rotating hairy black hole solutions in other non-Abelian theories such as Einstein-Yang-Mills-Higgs theory. For instance, we expect rotating black hole solutions with magnetic charge and with magnetic dipole hair [7, 6], Furthermore, the recent conjecture, that "any dyon solution with nonzero angular momentum necessarily contains an event horizon" 28 awaits investigation. 


\section{Appendix A: Ricci circularity conditions}

Let us demonstrate that the ansatz for the metric (8) satisfies the Ricci circularity conditions, Eq. (11).

The Killing vectors $\xi=\partial_{t}$ and $\eta=\partial_{\varphi}$ have components

$$
\begin{array}{ll}
\xi^{\mu}=(1,0,0,0), & \xi_{\mu}=\xi^{\nu} g_{\mu \nu}=\left(g_{t t}, 0,0, g_{t \varphi}\right), \\
\eta^{\mu}=(0,0,0,1), & \eta_{\mu}=\eta^{\nu} g_{\mu \nu}=\left(g_{\varphi t}, 0,0, g_{\varphi \varphi}\right) .
\end{array}
$$

Consider the Ricci circularity condition $\xi^{\mu} R_{\mu[\alpha} \xi_{\beta} \eta_{\gamma]}=0$, Eq. (11), where the square bracket denotes antisymmetrization. Hence, $\alpha \neq \beta \neq \gamma$. Thus $\xi^{\mu} R_{\mu[\alpha} \xi_{\beta} \eta_{\gamma]} \neq 0$ is only possible if either $\beta=0, \gamma=3$ or $\beta=3, \gamma=0$. In both cases $\alpha=1$ or $\alpha=2$.

For $\alpha=1$,

$$
\begin{aligned}
\xi^{\mu} R_{\mu[1} \xi_{0} \eta_{3]} & =R_{0[1} \xi_{0} \eta_{3]} \\
& =\frac{1}{6}\left(R_{01} \xi_{0} \eta_{3}+R_{00} \xi_{3} \eta_{1}+R_{03} \xi_{1} \eta_{0}-R_{03} \xi_{0} \eta_{1}-R_{00} \xi_{1} \eta_{3}-R_{01} \xi_{3} \eta_{0}\right) \\
& =\frac{1}{3} R_{01}\left(\xi_{0} \eta_{3}-\xi_{3} \eta_{0}\right) \\
& =\frac{1}{3} R_{01}\left(g_{t t} g_{\varphi \varphi}-g_{\varphi t}^{2}\right)
\end{aligned}
$$

and similarly, for $\alpha=2$,

$$
\xi^{\mu} R_{\mu[2} \xi_{0} \eta_{3]}=\frac{1}{3} R_{02}\left(g_{t t} g_{\varphi \varphi}-g_{\varphi t}^{2}\right)
$$

since all other components vanish due to $\xi_{1}=0, \xi_{2}=0$ and $\eta_{1}=0, \eta_{2}=0$.

On the other hand, the ansatz for the metric Eq. (8) yields

$$
R_{01}=R_{t r}=0, \quad R_{02}=R_{t \theta}=0 .
$$

Consequently, $\xi^{\mu} R_{\mu[\alpha} \xi_{\beta} \eta_{\gamma]}=0$.

Note that $R_{t r}=0, R_{t \theta}=0$ implies $G_{t r}=R_{t r}-1 / 2 g_{t r} R=0$ and $G_{t \theta}=R_{t \theta}-$ $1 / 2 g_{t \theta} R=0$, respectively. Therefore $T_{t r}=0$ and $T_{t \theta}=0$ is a necessary condition for the solutions of the Einstein equations. In a similar fashion it can be shown that the Ricci circularity condition $\eta^{\mu} R_{\mu[\alpha} \xi_{\beta} \eta_{\gamma]}=0$, Eq. (11), is satisfied by the metric (8) and implies $T_{\varphi r}=0$ and $T_{\varphi \theta}=0$. However, for the Ansatz (15) for the gauge field these conditions are fullfilled identically. Hence the solutions satisfy the Ricci circularity conditions. 


\section{Appendix B: Asymptotic expansion}

\subsection{General asymptotic expansion}

The asymptotic expansion of the fields may be obtained from the field equations and the corresponding boundary conditions. However, the process is rather involved. Indeed, the most natural assumption for the asymptotic $r$-dependence of the functions, i.e. polynomial, seems to be in contradiction with the presence of a non-vanishing electric charge. In view of that and inspired by perturbative results [12], we allowed for the presence of logarithms in the expansions. We then observed that, when keeping only a finite number of logarithmic terms, the charge was forced to vanish. By permitting an infinite number of such terms, however, it became possible to obtain a consistent expansion in the presence of an electric charge. This feature then suggested to include non-integer powers of $r$, with the exponents depending on the electric charge.

Numerically, we realized that there should be a splitting of some terms in the $1 / r$ Taylor series expansion of the static spherically symmetric case. However, the exponents of the new terms turned out to be very close to integer numbers, even though the behavior of the functions could not be described just by means of a $1 / r$ Taylor series. The procedure of how to compute this expansion was then clear.

First of all, we introduced a formal parameter $\epsilon$ in order to characterize terms of the order of $1 / r$, without assuming a Taylor series in $1 / r$ for the functions. Then we expanded all the functions in this formal parameter, the coefficients of such expansions depending on $r$ and $\theta$. Finally, we introduced those $\epsilon$ series into the system of field equations, taking into account the explicit dependence on $r$ in the equations by including $\epsilon$ appropriately. The last step was collecting coefficients in $\epsilon$ for each equation of the system, and solving the equations so formed, order by order in this formal parameter, keeping in mind the boundary conditions and the fact that the coefficients of the $\epsilon$ series had to behave consistently with the corresponding power of $\epsilon$.

Proceeding in this way, the expression for this asymptotic expansion is found to be:

$$
\begin{aligned}
f & =1-\frac{2 M}{x}+\frac{2 M^{2}+Q^{2}}{x^{2}}+o\left(\frac{1}{x^{2}}\right), \\
m & =1+\frac{C_{1}}{x^{2}}+\frac{Q^{2}-M^{2}-2 C_{1}}{x^{2}} \sin ^{2} \theta+o\left(\frac{1}{x^{2}}\right), \\
l & =1+\frac{C_{1}}{x^{2}}+o\left(\frac{1}{x^{2}}\right) \\
\omega & =-\frac{2 a M}{x^{2}}+\frac{6 a M^{2}+C_{2} Q}{x^{3}}+o\left(\frac{1}{x^{3}}\right), \\
H_{1} & =\left[\frac{2 C_{5}}{x^{2}}+\frac{8 C_{4}}{\beta-1} x^{-\frac{1}{2}(\beta-1)}-\frac{2 C_{2} C_{3}(\alpha+3)}{(\alpha+5) Q^{2}} x^{-\frac{1}{2}(\alpha+1)}\right] \sin \theta \cos \theta+o\left(\frac{1}{x^{2}}\right),
\end{aligned}
$$




$$
\begin{aligned}
& H_{2}=-1+C_{3} x^{-\frac{1}{2}(\alpha-1)}+\left[\frac{C_{5}}{x^{2}}+C_{4} x^{-\frac{1}{2}(\beta-1)}+\frac{C_{3}\left(\alpha^{2}+2 \alpha-11\right)}{2(\alpha+1)}\right. \\
& \left.\left(M-\frac{2 C_{2}(\alpha+3)}{(\alpha+5) Q^{2}}\right) x^{-\frac{1}{2}(\alpha+1)}\right]+\left[-\frac{2 C_{5}}{x^{2}}-2 C_{4} x^{-\frac{1}{2}(\beta-1)}\right. \\
& \left.+\frac{C_{2} C_{3}(\alpha+1)(\alpha+3)}{2(\alpha+5) Q^{2}} x^{-\frac{1}{2}(\alpha+1)}\right] \sin ^{2} \theta+o\left(\frac{1}{x^{2}}\right), \\
& H_{3}=\left(\frac{C_{2}}{x}+C_{3} x^{-\frac{1}{2}(\alpha-1)}\right) \sin \theta \cos \theta+\left[\frac{C_{3}\left(\alpha^{2}+2 \alpha-11\right)}{2(\alpha+1)}\right. \\
& \left(M-\frac{2 C_{2}(\alpha+3)}{(\alpha+5) Q^{2}}\right) x^{-\frac{1}{2}(\alpha+1)}+C_{4} x^{-\frac{1}{2}(\beta-1)}+\frac{3 C_{3}^{2}}{(\alpha+1)(\alpha-2)} x^{-(\alpha-1)} \\
& \left.+\frac{2 C_{5}+C_{2} M-3 a M Q}{2 x^{2}}\right] \sin \theta \cos \theta+o\left(\frac{1}{x^{2}}\right), \\
& H_{4}=-1+C_{3} x^{-\frac{1}{2}(\alpha-1)}-\left(\frac{C_{2}}{x}+C_{3} x^{-\frac{1}{2}(\alpha-1)}\right) \sin ^{2} \theta+\left[\frac{C_{3}\left(\alpha^{2}+2 \alpha-11\right)}{2(\alpha+1)}\right. \\
& \left.\left(M-\frac{2 C_{2}(\alpha+3)}{(\alpha+5) Q^{2}}\right) x^{-\frac{1}{2}(\alpha+1)}+C_{4} x^{-\frac{1}{2}(\beta-1)}+\frac{C_{5}}{x^{2}}\right] \\
& -\left[\frac{C_{3}\left(\alpha^{2}+2 \alpha-11\right)}{2(\alpha+1)}\left(M-\frac{2 C_{2}(\alpha+3)}{(\alpha+5) Q^{2}}\right) x^{-\frac{1}{2}(\alpha+1)}+C_{4} x^{-\frac{1}{2}(\beta-1)}\right. \\
& \left.+\frac{3 C_{3}^{2}}{(\alpha+1)(\alpha-2)} x^{-(\alpha-1)}+\frac{2 C_{5}+C_{2} M-3 a M Q}{2 x^{2}}\right] \sin ^{2} \theta+o\left(\frac{1}{x^{2}}\right), \\
& \bar{B}_{1}=\frac{Q \cos \theta}{x}-\frac{M Q \cos \theta}{x^{2}}+\left[\frac{2 C_{4}(\beta-5)}{(\beta-1) Q} x^{-\frac{1}{2}(\beta+1)}-\frac{4 C_{2} C_{3} Q}{(\alpha-3)(\alpha+5)} x^{-\frac{1}{2}(\alpha+3)}\right. \\
& \left.+\frac{C_{3}^{2}\left(\alpha^{2}-\alpha-8\right) Q}{\alpha(\alpha-1)(\alpha+2)(\alpha-3)} x^{-\alpha}+\frac{2 Q^{3}-C_{1} Q+4 M^{2} Q+4 C_{5} Q-2 C_{6}}{6 x^{3}}\right] \cos \theta \\
& +\left[\frac{C_{6}}{x^{3}}+\frac{C_{3}^{2} Q}{(\alpha+2)(\alpha-3)} x^{-\alpha}+\frac{8 C_{4} Q}{(\beta-1)(\beta+5)} x^{-\frac{1}{2}(\beta+1)}\right. \\
& \left.+\frac{4 C_{2} C_{3} Q}{(\alpha-3)(\alpha+5)} x^{-\frac{1}{2}(\alpha+3)}\right] \cos ^{3} \theta+o\left(\frac{1}{x^{3}}\right), \\
& \bar{B}_{2}=\frac{Q \sin \theta}{x}-\frac{M Q \sin \theta}{x^{2}}+\left[\frac{2 Q^{3}-C_{1} Q+4 M^{2} Q-2 C_{5} Q-2 C_{6}-24 a M}{6 x^{3}}\right. \\
& \left.+\frac{C_{3}^{2}\left(\alpha^{2}-\alpha-8\right) Q}{\alpha(\alpha-1)(\alpha+2)(\alpha-3)} x^{-\alpha}\right] \sin \theta+\left[\frac{C_{6}}{x^{3}}+\frac{C_{3}^{2} Q}{(\alpha+2)(\alpha-3)} x^{-\alpha}\right. \\
& \left.+\frac{4 C_{2} C_{3} Q}{(\alpha-3)(\alpha+5)} x^{-\frac{1}{2}(\alpha+3)}-\frac{2 C_{4}(\beta-5)}{(\beta-1) Q} x^{-\frac{1}{2}(\beta+1)}\right] \sin \theta \cos ^{2} \theta+o\left(\frac{1}{x^{3}}\right)
\end{aligned}
$$

with the notation of section 3.1. 


\subsection{Asymptotic expansion: relation with static case}

Here we show how the previous asymptotic expansions reduce to the ones corresponding to the static case in the limit of vanishing charge. Moreover, as these are nonperturbative expansions, they must include the perturbative expansions reported in [12]. This is indeed the case. In order to perform perturbative expansions in terms of the electric charge, we must recall that, for a fixed value of the horizon radius, all the parameters in these expansions are functions of $Q$. Due to the presence of $Q$ in some denominators, the dependence on $Q$ of these coefficients has to be singular, in such a way that the resulting series in $Q$ turns out to be regular. The behavior of the constants as functions of $Q$ reads:

$$
\begin{aligned}
M & =M_{0}+Q^{2} K_{0}(Q), \\
C_{1} & =-\frac{1}{2} M_{0}^{2}+Q^{2} K_{1}(Q) \\
C_{2} & =-b+Q^{2} K_{2}(Q) \\
C_{3} & =b+Q^{2} K_{3}(Q) \\
C_{4} & =-\frac{5}{4} \frac{b^{2}}{Q^{2}}+K_{4}(Q) \\
C_{5} & =\frac{1}{2} \frac{b^{2}}{Q^{2}}+\left[\frac{7}{48} b^{2}+\frac{3}{4} b\left(K_{2}(Q)-K_{3}(Q)\right)-\frac{1}{5} K_{4}(Q)\right]+Q K_{5}(Q), \\
C_{6} & =-\frac{1}{5} \frac{b^{2}}{Q^{2}}+Q\left[\frac{1}{16} b^{2}+\frac{3}{4} b K_{2}(Q)-\frac{3}{20} b K_{3}(Q)-\frac{1}{5} K_{4}(Q)\right]+Q^{2} K_{6}(Q), \\
a & =Q K_{7}(Q)
\end{aligned}
$$

where $M_{0}$ is the dimensionless mass of the static spherically symmetric solution, $b$ is the parameter of the gauge field for such a limiting solution, and $K_{1}, \ldots, K_{7}$ are regular functions of $Q$. Introducing these relations in (118) and expanding the result in $Q$, we recover the perturbative expansions given in [12]:

$$
\begin{aligned}
f & =1-\frac{2 M^{\prime}}{x}+\frac{2 M^{\prime 2}}{x^{2}}+O\left(Q^{2}\right) \\
m & =1-\frac{M^{\prime 2}}{2 x^{2}}+O\left(Q^{2}\right) \\
l & =1-\frac{M^{\prime 2}}{2 x^{2}}+O\left(Q^{2}\right) \\
\omega & =-\frac{2 J^{\prime} M^{\prime}}{x^{2}}+\frac{6 J^{\prime} M^{\prime 2}-a^{\prime} Q^{\prime}}{x^{3}} \\
H_{1} & =O\left(Q^{2}\right) \\
H_{2} & =-1+\frac{a^{\prime}}{x}+\frac{2 a^{\prime} M^{\prime}-3 a^{\prime 2}}{4 x^{2}}+O\left(Q^{2}\right)
\end{aligned}
$$




$$
\begin{aligned}
H_{3}= & O\left(Q^{2}\right) \\
H_{4}= & -1+\frac{a^{\prime}}{x}+\frac{2 a^{\prime} M^{\prime}-3 a^{\prime 2}}{4 x^{2}}+O\left(Q^{2}\right), \\
\bar{B}_{1}= & \frac{Q^{\prime} \cos \theta}{x}-\frac{M^{\prime} Q^{\prime} \cos \theta}{x^{2}}+\frac{1}{60 x^{3}} \cos \theta\left(40 a^{\prime 2} Q^{\prime}+225 M^{\prime 2} Q^{\prime}+120 c_{4}^{\prime}\right. \\
& \left.-8 a^{\prime 2} Q^{\prime} \ln x\right)+O\left(Q^{2}\right), \\
\bar{B}_{2}= & \frac{Q^{\prime} \sin \theta}{x}-\frac{M^{\prime} Q^{\prime} \sin \theta}{x^{2}}-\frac{1}{60 x^{3}} \sin \theta\left(45 M^{\prime 2} Q^{\prime}+240 J^{\prime} M^{\prime}+60 c_{4}^{\prime}\right. \\
& \left.-4 a^{\prime 2} Q^{\prime} \ln x\right)+O\left(Q^{2}\right),
\end{aligned}
$$

where primes denote the notation of Volkov and Straumann, which is related to our notation by

$$
\begin{aligned}
M^{\prime} & =M_{0} \\
a^{\prime} & =b \\
Q^{\prime} & =Q \\
J^{\prime} & =Q K_{7}(0) \\
c_{4}^{\prime} & =\frac{Q}{200}\left[100 b\left(K_{2}(0)-K_{3}(0)\right)-31 b^{2}-300 M_{0}^{2}-80 K_{4}(0)\right] .
\end{aligned}
$$

\subsection{Asymptotic expansion: embedded Kerr-Newman solutions}

For comparison, we present the asymptotic expansion also for the embedded KerrNewman solutions for our choice of coordinates and gauge. The expansion reads

$$
\begin{aligned}
f & =1-\frac{2 M}{x}+\frac{2 M^{2}+Q^{2}+P^{2}}{x^{2}}+O\left(\frac{1}{x^{3}}\right) \\
l & =1+\frac{a^{2}-M^{2}+Q^{2}+P^{2}}{2 x^{2}}+O\left(\frac{1}{x^{3}}\right) \\
m & =1+\frac{a^{2}-M^{2}+Q^{2}+P^{2}}{2 x^{2}}-\frac{a^{2} \sin ^{2} \theta}{x^{2}}+O\left(\frac{1}{x^{3}}\right), \\
\omega & =\frac{2 a M}{x^{2}}-\frac{a\left(Q^{2}+P^{2}+6 M^{2}\right)}{x^{3}}+O\left(\frac{1}{x^{4}}\right) \\
H_{1} & =0 \\
H_{2} & =0 \\
H_{3} & =-(1+P) \cot \theta-\frac{a Q \sin \theta}{x}-\frac{a \sin \theta(a P \cos \theta-M Q)}{x^{2}}+O\left(\frac{1}{x^{3}}\right), \\
H_{4} & =0, \\
\bar{B}_{1} & =\frac{Q}{x}+\frac{a P \cos \theta-M Q}{x^{2}}+O\left(\frac{1}{x^{3}}\right),
\end{aligned}
$$




$$
\bar{B}_{2}=\frac{2 a M \sin \theta}{x^{3}}+O\left(\frac{1}{x^{4}}\right),
$$

where $a$ is the angular momentum per unit mass, $M$ is the mass, $Q$ is the electric charge, $\left(Q^{2}=Q^{a} Q^{a}\right)$, and $P$ is the magnetic charge, $\left(P^{2}=P^{a} P^{a}\right)$.

\section{Appendix C: Expansion at the horizon}

We here first motivate our choice of boundary conditions at the horizon. Then we give the full expansion of the metric and gauge field functions at the horizon and relate this general expansion with the static case.

\subsection{Boundary conditions at the horizon}

Let us begin by noting that the ansatz of the gauge field has the property [13, 28]

$$
\partial_{\varphi} A_{\mu}=D_{\mu} u
$$

with $u=\tau_{z} / 2$. The components $F_{\mu \varphi}$ can be expressed as

$$
F_{\mu \varphi}=D_{\mu} W
$$

with

$$
W=A_{\varphi}-u .
$$

$W$ transforms as a scalar doublet under gauge transformations $U=\exp \left(i \Gamma \tau_{\phi} / 2\right)$, Eq. (20). Using the definition of $W$, Eq. (132), we find for the component $A_{t}$ of the gauge field

$$
A_{t}=\Psi+\frac{\omega}{r} \frac{\tau_{z}}{2}+\frac{\omega}{r} W=\hat{\Psi}+\frac{\omega}{r} W
$$

with

$$
\hat{\Psi}=\Psi+\frac{\omega}{r} \frac{\tau_{z}}{2} .
$$

Thus $\hat{\Psi}$ also transforms as a scalar doublet under gauge transformations.

To discuss the behaviour of the solutions at the horizon it is convenient to rewrite $\Psi$ and $A_{\varphi}$ as

$$
\Psi=-\frac{\tilde{B}_{1}}{x_{\mathrm{H}}} \frac{\tau_{z}}{2}+\frac{\tilde{B}_{2}}{x_{\mathrm{H}}} \frac{\tau_{\rho}}{2}, \quad A_{\varphi}=-\sin \theta\left[\tilde{H}_{4} \frac{\tau_{z}}{2}-\tilde{H}_{3} \frac{\tau_{\rho}}{2}\right] .
$$

This yields for the scalar doublets, $W$ and $\hat{\Psi}$, Eqs. (132), (134),

$$
\begin{aligned}
W & =\sin \theta \tilde{H}_{3} \frac{\tau_{\rho}}{2}-\left(\sin \theta \tilde{H}_{4}+1\right) \frac{\tau_{z}}{2}, \\
\hat{\Psi} & =-\left(\frac{\tilde{B}_{1}}{x_{\mathrm{H}}}-\frac{\omega}{x}\right) \frac{\tau_{z}}{2}+\frac{\tilde{B}_{2}}{x_{\mathrm{H}}} \frac{\tau_{\rho}}{2} .
\end{aligned}
$$


We assume (see below) that near the horizon the metric functions can be expanded as

$$
f=f_{2} \delta^{2}+O\left(\delta^{3}\right), \quad m=m_{2} \delta^{2}+O\left(\delta^{3}\right), \quad l=l_{2} \delta^{2}+O\left(\delta^{3}\right), \quad \omega=\omega_{\mathrm{H}}+\omega_{1} \delta+O\left(\delta^{2}\right),
$$

where $\delta=\left(x-x_{\mathrm{H}}\right) / x_{\mathrm{H}}$ and $\omega_{\mathrm{H}}$ is a constant. For the gauge field functions we assume an expansion in the form

$$
\begin{aligned}
& H_{1}=H_{11} \delta+O\left(\delta^{2}\right), \\
& H_{2}=H_{20}+H_{21} \delta+O\left(\delta^{2}\right), \\
& \tilde{H}_{3}=\tilde{H}_{30}+\tilde{H}_{31} \delta+O\left(\delta^{2}\right), \\
& \tilde{H}_{4}=\tilde{H}_{40}+\tilde{H}_{41} \delta+O\left(\delta^{2}\right), \\
& \tilde{B}_{1}=\tilde{B}_{10}+\tilde{B}_{11} \delta+O\left(\delta^{2}\right), \\
& \tilde{B}_{2}=\tilde{B}_{20}+\tilde{B}_{21} \delta+O\left(\delta^{2}\right),
\end{aligned}
$$

where $\left.H_{1}\right|_{x=x_{\mathrm{H}}}=0$ fixes the gauge freedom.

Let us write the field equations as

$$
E^{\mu}=\frac{1}{\sqrt{-g}} D_{\nu}\left(\sqrt{-g} F^{\nu \mu}\right)=0, \quad E_{\mu \nu}=G_{\mu \nu}-8 \pi G T_{\mu \nu}=0 .
$$

The expansion of $E^{t}$ at the horizon yields

$$
\tilde{B}_{11}=\tilde{H}_{40}\left(\omega_{\mathrm{H}}-\omega_{1}\right) \sin \theta, \quad \tilde{B}_{21}=\tilde{H}_{30}\left(\omega_{\mathrm{H}}-\omega_{1}\right) \sin \theta .
$$

With this result the expansion of $E^{\varphi}$ leads to the conditions

$$
\begin{aligned}
{\left[\left(\tilde{B}_{10}-\omega_{\mathrm{H}}\right) \tilde{H}_{30} \sin \theta-\tilde{B}_{20}\left(\tilde{H}_{40} \sin \theta+1\right)\right] \tilde{B}_{20} } & =0, \\
{\left[\left(\tilde{B}_{10}-\omega_{\mathrm{H}}\right) \tilde{H}_{30} \sin \theta-\tilde{B}_{20}\left(\tilde{H}_{40} \sin \theta+1\right)\right]\left(\tilde{B}_{10}-\omega_{\mathrm{H}}\right) } & =0 .
\end{aligned}
$$

In terms of $W$ and $\hat{\Psi}$ these conditions are equivalent to

$$
\left.[\hat{\Psi},[\hat{\Psi}, W]]\right|_{x=x_{\mathrm{H}}}=\left.0 \Longleftrightarrow[\hat{\Psi}, W]\right|_{x=x_{\mathrm{H}}}=\left.0 \Longleftrightarrow F_{t \varphi}\right|_{x=x_{\mathrm{H}}}=0 .
$$

We now assume that the electro-static potential $\Psi$ is constant at the horizon, Eq. (55), i. e. $\tilde{B}_{10}=$ const and $\tilde{B}_{20}=0$.

To discuss the boundary conditions (143), let us first assume $\left.\hat{\Psi}\right|_{x=x_{\mathrm{H}}} \neq 0$ and $\left.W\right|_{x=x_{\mathrm{H}}}=\left.\lambda \hat{\Psi}\right|_{x=x_{\mathrm{H}}}$, for some function $\lambda(\theta)$. In this case the expansion yields for the gauge potential

$$
A_{\mu} d x^{\mu}=\left\{-\left[\frac{\tilde{B}_{10}}{x_{\mathrm{H}}}+O\left(\delta^{2}\right)\right] d t+\left[\frac{\lambda}{x_{\mathrm{H}}}\left(\tilde{B}_{10}-\omega_{\mathrm{H}}\right)+1+O\left(\delta^{2}\right)\right]\left(d \varphi+\frac{\omega}{x} d t\right)\right\} \frac{\tau_{z}}{2}+O\left(\delta^{4}\right) .
$$


The functions $H_{1}, 1-H_{2}, \tilde{H}_{3}$ and $\tilde{B}_{2}$ vanish at least up to order $O\left(\delta^{4}\right)$, indicating that we find only embedded Abelian solutions for $\left.\hat{\Psi}\right|_{x=x_{\mathrm{H}}} \neq 0$.

Assuming $\left.\hat{\Psi}\right|_{x=x_{\mathrm{H}}}=0$, however, does not imply restrictions on $H_{20}, \tilde{H}_{30}$ and $\tilde{H}_{40}$. In this case the expansion yields $H_{21}=\tilde{H}_{31}=\tilde{H}_{41}=0$ and $\omega_{1}=\omega_{\mathrm{H}}$. The last condition implies $\tilde{B}_{11}=\tilde{B}_{21}=0$. In this case non-Abelian solutions are possible.

\subsection{General expansion at the horizon}

Here we present the expansion of the functions of the stationary axially symmetric black hole solutions at the horizon $x_{\mathrm{H}}$ in powers of $\delta$. These expansions can be obtained from the regularity conditions imposed on the Einstein equations and the matter field equations:

$$
\begin{aligned}
f(\delta, \theta)= & \delta^{2} f_{2}\left\{1-\delta+\frac{\delta^{2}}{24}\left[\frac { 1 } { x _ { \mathrm { H } } ^ { 2 } } \frac { f _ { 2 } } { l _ { 2 } } \left[2 4 \operatorname { c o t } \theta \left[-\left(-H_{30, \theta}+1-H_{20}{ }^{2}\right) H_{30}\right.\right.\right.\right. \\
& \left.-H_{20} H_{40, \theta}+H_{40} H_{40, \theta}\right]+12\left(H_{20}^{2}\left(H_{30}^{2}+H_{40}^{2}-1\right)+\frac{\left(H_{20}-H_{40}\right)^{2}+H_{30}^{2}}{\sin ^{2} \theta}\right. \\
& -\left(H_{30}^{2}+H_{40}^{2}\right)+2 H_{20}\left(-H_{30} H_{40, \theta}+H_{40} H_{30, \theta}\right)+1-2 H_{30, \theta}+H_{30, \theta}^{2} \\
& \left.\left.+H_{40, \theta}^{2}\right)\right]-2 \cot \theta\left(3 \frac{f_{2, \theta}}{f_{2}}-2 \frac{l_{2, \theta}}{l_{2}}\right)-\left(3 \frac{f_{2, \theta}}{f_{2}} \frac{l_{2, \theta}}{l_{2}}+6 \frac{f_{2, \theta \theta}}{f_{2}}+\left(\frac{l_{2, \theta}}{l_{2}}\right)^{2}\right. \\
& \left.-2 \frac{l_{2, \theta \theta}}{l_{2}}-18-6\left(\frac{f_{2, \theta}}{f_{2}}\right)^{2}\right)-\frac{24}{f_{2}}\left[4 \sin \theta \frac{\omega_{2}}{x_{\mathrm{H}}}\left(H_{30} B_{12}+\left(1-H_{40}\right) B_{22}\right)\right. \\
& \left.\left.\left.-2\left(B_{12}^{2}+B_{22}^{2}\right)-\sin ^{2} \theta \frac{\omega_{2}^{2}}{x_{\mathrm{H}}^{2} f_{2}}\left(x_{\mathrm{H}}^{2} l_{2}+2 f_{2}\left(H_{30}^{2}+\left(1-H_{40}\right)^{2}\right)\right)\right]\right]\right\}+O\left(\delta^{5}\right), \\
m(\delta, \theta)= & \delta^{2} m_{2}\left\{1-3 \delta+\frac{\delta^{2}}{24}\left[150-4 \frac{l_{2, \theta \theta}}{l_{2}}+2\left(\frac{l_{2, \theta}}{l_{2}}\right)^{2}+3 \frac{l_{2, \theta}}{l_{2}} \frac{m_{2, \theta}}{m_{2}}-6 \frac{m_{2, \theta \theta}}{m_{2}}\right.\right. \\
& \left.\left.+6\left(\frac{m_{2, \theta}}{m_{2}}\right)^{2}-6\left(\frac{f_{2, \theta}}{f_{2}}\right)^{2}+2 \cot \theta\left(3 \frac{m_{2, \theta}}{m_{2}}-4 \frac{l_{2, \theta}}{l_{2}}\right)+24 \sin ^{2} \theta \frac{l_{2} \omega_{2}^{2}}{f_{2}^{2}}\right]\right\}+O\left(\delta^{5}\right), \\
l(\delta, \theta)= & \delta^{2} l_{2}\left\{1-3 \delta+\frac{\delta^{2}}{12}\left[\left(\frac{l_{2, \theta}}{l_{2}}\right)^{2}-2 \frac{l_{2, \theta \theta}}{l_{2}}+75-4 \cot \theta \frac{l_{2, \theta}}{l_{2}}\right]\right\}+O\left(\delta^{5}\right), \\
\omega(\delta, \theta)= & \omega_{\mathrm{H}}(1+\delta)+\delta^{2} \omega_{2}+O\left(\delta^{4}\right), \\
H_{1}(\delta, \theta)= & \delta\left(1-\frac{1}{2} \delta\right) H_{11}+O\left(\delta^{3}\right) \\
H_{2}(\delta, \theta)= & H_{20}+\frac{\delta^{2}}{4}\left[\frac { m _ { 2 } } { l _ { 2 } } \left(H_{20}\left(H_{30}^{2}+H_{40}^{2}-1\right)-H_{30} H_{40, \theta}+H_{40} H_{30, \theta}+\frac{H_{20}-H_{40}}{\sin ^{2} \theta}\right.\right. \\
& \left.\left.-\cot \theta\left(-2 H_{20} H_{30}+H_{40, \theta}\right)\right)-\left(H_{11, \theta}+H_{20, \theta \theta}\right)\right]+O\left(\delta^{3}\right),
\end{aligned}
$$




$$
\begin{aligned}
H_{3}(\delta, \theta)= & H_{30}-\frac{\delta^{2}}{8}\left[-\left(2 \frac{f_{2, \theta}}{f_{2}}-\frac{l_{2, \theta}}{l_{2}}\right)\left(1-H_{40} H_{20}-H_{30, \theta}-\cot \theta H_{30}\right)\right. \\
& -2 \cot \theta H_{20}\left(H_{20}-H_{40}\right)+2 H_{30, \theta \theta}+4 H_{20} H_{40, \theta}-2\left(\frac{H_{30}}{\sin ^{2} \theta}-2 \cot \theta H_{30, \theta}\right) \\
& \left.-2 H_{30} H_{20}^{2}-2 H_{40}\left(2 H_{11}-H_{20, \theta}\right)-8 \sin \theta \frac{l_{2} \omega_{2}}{f_{2}^{2}}\left(x_{\mathrm{H}} B_{12}-\sin \theta \omega_{2} H_{30}\right)\right]+O\left(\delta^{3}\right), \\
H_{4}(\delta, \theta)= & H_{40}-\frac{\delta^{2}}{8}\left[\left(2 \frac{f_{2, \theta}}{f_{2}}-\frac{l_{2, \theta}}{l_{2}}\right)\left[H_{40, \theta}-H_{20} H_{30}-\cot \theta\left(H_{20}-H_{40}\right)\right]\right. \\
& +H_{20}\left(-4 H_{30, \theta}+2\right)+2\left[H_{30}\left(2 H_{11}-H_{20, \theta}\right)+H_{40, \theta \theta}-H_{40} H_{20}^{2}\right] \\
& +2 \frac{H_{20}-H_{40}}{\sin ^{2} \theta}-2 \cot \theta\left(-2 H_{11}-H_{40, \theta}+H_{20} H_{30}+H_{20, \theta}\right) \\
& \left.+8 \sin \theta \frac{l_{2} \omega_{2}}{f_{2}^{2}}\left[x_{\mathrm{H}} B_{22}-\sin \theta \omega_{2}\left(1-H_{40}\right)\right]\right]+O\left(\delta^{3}\right), \\
\bar{B}_{1}(\delta, \theta)= & -\frac{\omega_{\mathrm{H}} \cos \theta}{x_{\mathrm{H}}}+\delta^{2}(1-\delta) B_{12}+O\left(\delta^{4}\right), \\
\bar{B}_{2}(\delta, \theta)= & \frac{\omega_{\mathrm{H}} \sin \theta}{x_{\mathrm{H}}}+\delta^{2}(1-\delta) B_{22}+O\left(\delta^{4}\right) .
\end{aligned}
$$

$B_{12}$ and $B_{22}$ are functions of $\theta$. Relations (76) and (77) also hold.

\subsection{Expansion: relation with static case}

The general expansion at the horizon includes the expansion for the static case. The static limit corresponds to setting $\omega_{\mathrm{H}}=\omega_{2}=B_{12}=B_{22}=H_{11}=H_{31}=0, m_{2}=l_{2}$, and $H_{40}=H_{20}$; in addition, $f_{2}, l_{2}$, and $H_{20}$ become constant. The expansion reads

$$
\begin{aligned}
f(\delta) & =\delta^{2} f_{2}\left\{1-\delta+\frac{\delta^{2}}{4}\left[\frac{2}{x_{\mathrm{H}}^{2}} \frac{f_{2}}{l_{2}}\left(H_{20}^{2}-1\right)^{2}+3\right]\right\}+O\left(\delta^{5}\right), \\
l(\delta) & =\delta^{2} l_{2}\left(1-3 \delta+\frac{25}{4} \delta^{2}\right)+O\left(\delta^{5}\right), \\
H_{2}(\delta) & =H_{20}\left[1+\frac{\delta^{2}}{4}\left(H_{20}^{2}-1\right)\right]+O\left(\delta^{3}\right),
\end{aligned}
$$

recovering the known expressions for the behavior at the horizon of the static spherically symmetric black hole solutions.

\subsection{Expansion: embedded Kerr-Newman solutions}

For comparison, we present the expansion at the horizon also for the embedded Kerr-

Newman solutions for our choice of coordinates and gauge. The expansion reads 


$$
\begin{aligned}
& f=\frac{4 x_{\mathrm{H}}^{2}\left[\left(M+2 x_{\mathrm{H}}\right)^{2}+a^{2} \cos ^{2} \theta\right]}{\left[2 M\left(M+2 x_{\mathrm{H}}\right)-\left(Q^{2}+P^{2}\right)\right]^{2}} \delta^{2}(1-\delta)+O\left(\delta^{4}\right), \\
& m=\frac{4\left[\left(M+2 x_{\mathrm{H}}\right)^{2}+a^{2} \cos ^{2} \theta\right]^{2}}{\left[2 M\left(M+2 x_{\mathrm{H}}\right)-\left(Q^{2}+P^{2}\right)\right]^{2}} \delta^{2}(1-3 \delta)+O\left(\delta^{4}\right), \\
& l=4 \delta^{2}(1-3 \delta)+O\left(\delta^{4}\right), \\
& \omega=\frac{a x_{\mathrm{H}}}{2 M\left(M+2 x_{\mathrm{H}}\right)-\left(Q^{2}+P^{2}\right)}(1+\delta) \\
& -\frac{2 a x_{\mathrm{H}}^{2}}{\left[2 M\left(M+2 x_{\mathrm{H}}\right)-\left(Q^{2}+P^{2}\right)\right]^{3}}\left\{( M + 2 x _ { \mathrm { H } } ) \left[2\left(M+2 x_{\mathrm{H}}\right)\left(M+x_{\mathrm{H}}\right)\right.\right. \\
& \left.\left.-\left(Q^{2}+P^{2}\right)\right]+2 a^{2} x_{\mathrm{H}} \cos ^{2} \theta\right\} \delta^{2}+O\left(\delta^{3}\right), \\
& H_{1}=0 \text {, } \\
& H_{2}=0 \text {, } \\
& H_{3}=-\frac{1}{\left(M+2 x_{\mathrm{H}}\right)^{2}+a^{2} \cos ^{2} \theta}\left\{\operatorname { c o t } \theta \left[\left(M+2 x_{\mathrm{H}}\right)^{2}+P\left[2 M\left(M+2 x_{\mathrm{H}}\right)\right.\right.\right. \\
& \left.\left.\left.-\left(Q^{2}+P^{2}\right)\right]+a^{2} \cos ^{2} \theta\right]+a Q\left(M+2 x_{\mathrm{H}}\right) \sin \theta\right\} \\
& -\frac{a \sin \theta}{2\left[\left(M+2 x_{\mathrm{H}}\right)^{2}+a^{2} \cos ^{2} \theta\right]^{2}}\left[2 a^{2} Q x_{\mathrm{H}} \cos ^{2} \theta-4 a P x_{\mathrm{H}}\left(M+2 x_{\mathrm{H}}\right) \cos \theta\right. \\
& \left.-2 Q x_{\mathrm{H}}\left(M+2 x_{\mathrm{H}}\right)^{2}\right] \delta^{2}+O\left(\delta^{3}\right), \\
& H_{4}=0 \text {, } \\
& \bar{B}_{1}=\frac{Q\left(M+2 x_{\mathrm{H}}\right)-a \cos \theta}{2 M\left(M+2 x_{\mathrm{H}}\right)-\left(Q^{2}+P^{2}\right)} \\
& -\frac{1}{2\left[2 M\left(M+2 x_{\mathrm{H}}\right)-\left(Q^{2}+P^{2}\right)\right]^{3}}\left\{2 Q x _ { \mathrm { H } } \left[-\left[M\left(M+2 x_{\mathrm{H}}\right)-\left(Q^{2}+P^{2}\right)\right]^{2}\right.\right. \\
& \left.+\left(M+2 x_{\mathrm{H}}\right)^{2}\left[\left(M+2 x_{\mathrm{H}}\right)^{2}+4 x_{\mathrm{H}}^{2}\right]\right]-4 a x_{\mathrm{H}}\left[( M + 2 x _ { \mathrm { H } } ) \left[4 M P x_{\mathrm{H}}\right.\right. \\
& \left.\left.-\left(Q^{2}+P^{2}\right)\right]-2 P x_{\mathrm{H}}\left(Q^{2}+P^{2}\right)+2\left(M+2 x_{\mathrm{H}}\right)^{2}\left(M+x_{\mathrm{H}}\right)\right] \cos \theta \\
& \left.+8 a^{2} Q x_{\mathrm{H}}^{2}\left(M+2 x_{\mathrm{H}}\right) \cos ^{2} \theta-8 a^{3} x_{\mathrm{H}}^{2} \cos ^{3} \theta\right\} \delta^{2}+O\left(\delta^{3}\right) \text {, } \\
& \bar{B}_{2}=\frac{a \sin \theta}{2 M\left(M+2 x_{\mathrm{H}}\right)-\left(Q^{2}+P^{2}\right)} \\
& -\frac{2 a x_{\mathrm{H}} \sin \theta}{\left[2 M\left(M+2 x_{\mathrm{H}}\right)-\left(Q^{2}+P^{2}\right)\right]^{3}}\left\{\left(M+4 x_{\mathrm{H}}\right)\left[2 M\left(M+2 x_{\mathrm{H}}\right)-\left(Q^{2}+P^{2}\right)\right]\right. \\
& \left.-2 a^{2} x_{\mathrm{H}} \sin ^{2} \theta\right\} \delta^{2}+O\left(\delta^{3}\right) \text {, }
\end{aligned}
$$

where

$$
x_{\mathrm{H}}=\frac{1}{2} \sqrt{M^{2}-\left(a^{2}+Q^{2}+P^{2}\right)},
$$




$$
\delta=\frac{x}{x_{\mathrm{H}}}-1 .
$$




\section{References}

[1] W. Israel, Commun. Math. Phys. 8 (1968) 245;

D. C. Robinson, Phys. Rev. Lett. 34 (1975) 905;

P. Mazur, J. Phys. A15 (1982) 3173.

[2] M. Heusler, Black Hole Uniqueness Theorems, (Cambrigde University Press, 1996).

[3] see e.g. M. S. Volkov and D. V. Gal'tsov, Phys. Rept. 319 (1999) 1;

D. V. Gal'tsov, hep-th/0112038, Proceedings of the 16th International Conference on General Relativity and Gravitation, July 2001, Durban, South Africa

[4] M. S. Volkov and D. V. Galt'sov, Sov. J. Nucl. Phys. 51 (1990) 747;

P. Bizon, Phys. Rev. Lett. 64 (1990) 2844;

H. P. Künzle and A. K. M. Masoud-ul-Alam, J. Math. Phys. 31 (1990) 928.

[5] B. Kleihaus and J. Kunz, Phys. Rev. Lett. 79 (1997) 1595;

B. Kleihaus and J. Kunz, Phys. Rev. D57 (1998) 6138.

[6] B. Kleihaus and J. Kunz, Phys. Rev. Lett. 85 (2000) 2430;

B. Kleihaus and J. Kunz, Phys. Lett. B494 (2000) 130.

[7] B. Hartmann, B. Kleihaus, and J. Kunz, Phys. Rev. Lett. 86, 1423 (2001);

B. Hartmann, B. Kleihaus, and J. Kunz, Phys. Rev. D65 (2002) 0024027.

[8] D. Sudarsky and R. M. Wald, Phys. Rev. D46 (1992) 1453;

D. Sudarsky and R. M. Wald, Phys. Rev. D47 (1993) 5209.

[9] see e. g. D. Kramer, H. Stephani, E. Herlt, and M. MacCallum, Exact Solutions of Einstein's Field Equations, Ch. 17 (Cambridge University Press, Cambridge, 1980)

[10] A. Papapetrou, Ann. Inst. H. Poincare A4 1966 83;

B. Carter, J. Math. Phys. 10 (1969) 70.

[11] M. Heusler and N. Straumann, Class. Quant. Grav. 10, (1993) 21.

[12] M. S. Volkov and N. Straumann, Phys. Rev. Lett. 79 (1997) 1428.

[13] D. V. Gal'tsov, Einstein-Yang-Mills solitons: towards new degrees of freedom, gr-qc/9808002.

[14] D. V. Gal'tsov and A. A. Ershov, Phys. Lett. 138 A (1989) 160. 
[15] O. Brodbeck, M. Heusler, N. Straumann and M. S. Volkov, Phys. Rev. Lett. 79 (1997) 4310;

O. Brodbeck and M. Heusler, Phys. Rev. D56 (1997) 6278.

[16] B. Kleihaus and J. Kunz, Phys. Rev. Lett. 86 (2001) 3704.

[17] A. Corichi, and D. Sudarsky, Phys. Rev. D61 (2000) 101501;

A. Corichi, U. Nucamendi, and D. Sudarsky, Phys. Rev. D62 (2000) 044046.

[18] B. Kleihaus and J. Kunz, Phys. Rev. Lett. 78 (1997) 2527;

B. Kleihaus and J. Kunz, Phys. Rev. D57 (1998) 834.

[19] R. M. Wald, General Relativity (University of Chicago Press, Chicago, 1984)

[20] J. N. Islam, Rotating fields in general relativity (Cambridge University Press, Cambridge, 1985)

[21] A. Ashtekar, Ch. Beetle and J. Lewandowski, Phys. Rev. D64 (2001) 044016.

[22] M. J. Perry, Phys. Lett. B71 (1977) 234.

[23] When both electric and magnetic non-Abelian charge is present, the vectors must be parallel to each other, i.e. $Q^{a}=\lambda P^{a}$ with $\lambda$ constant [22].

[24] Since the gauge fields wind $n$ times around, while the azimuthal angle $\varphi$ covers the full trigonometric circle once, we refer to the integer $n$ as the winding number of the solutions.

[25] B. Kleihaus, J. Kunz, A. Sood and M. Wirschins, Phys. Rev. D58 (1998) 084006.

[26] The limiting solution corresponds to an embedded Reissner-Nordstrøm black hole with magnetic charge $|P|=n$ for horizon radius $\tilde{x}_{\mathrm{H}}>1$, where $\tilde{x}$ represents a Schwarzschild-like radial coordinate. [3, 5]. For $\tilde{x}_{\mathrm{H}}<1$, the limiting solution also corresponds to an embedded Reissner-Nordstrøm black hole with magnetic charge $|P|=n$ in the region $1<\tilde{x}$, but contains an oscillating non-Abelian part in the region $\tilde{x}_{\mathrm{H}}<\tilde{x}<1$ [3], 5].

[27] W. Schönauer and R. Weiß, J. Comput. Appl. Math. 27, 279 (1989) 279; M. Schauder, R. Weiß and W. Schönauer, The CADSOL Program Package, Universität Karlsruhe, Interner Bericht Nr. 46/92 (1992).

[28] J. J. van der Bij and E. Radu, On rotating regular nonabelian solutions, Int. J. Mod. Phys. A17 (2002) 1477.

[29] B. Kleihaus, J. Kunz, F. Navarro, in preparation. 
Fig. 1a

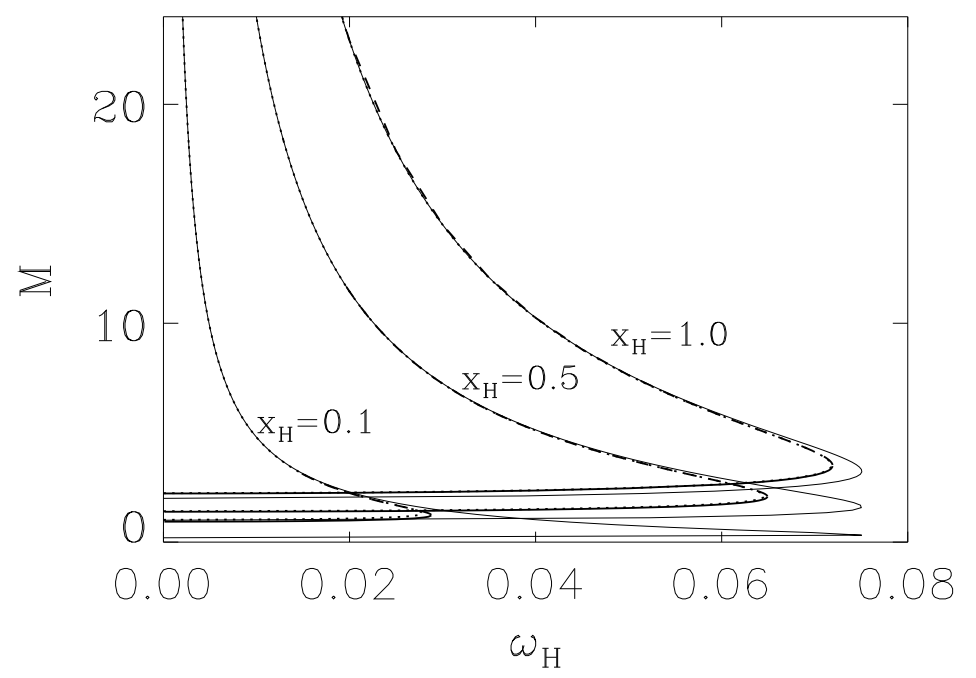

Figure 1a: The dimensionless mass $M$ is shown as a function of $\omega_{\mathrm{H}}$ for node number $k=1$ and $x_{\mathrm{H}}=1.0,0.5$ and 0.1 . For the same values of parameters the dimensionless mass of the Kerr solution (thin solid) and the Kerr-Newman solution (dotted) for $Q=0$ and $|P|=1$ are also shown. 
Fig. 1b

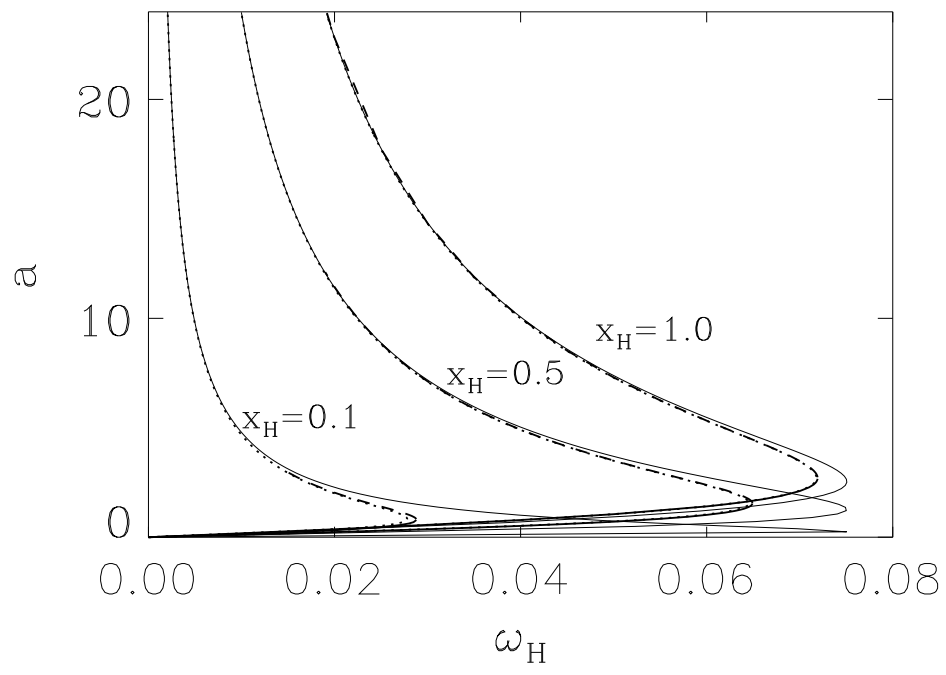

Figure 1b: Same as Fig. 1a for the ratio $a=J / M$.

Fig. 1c

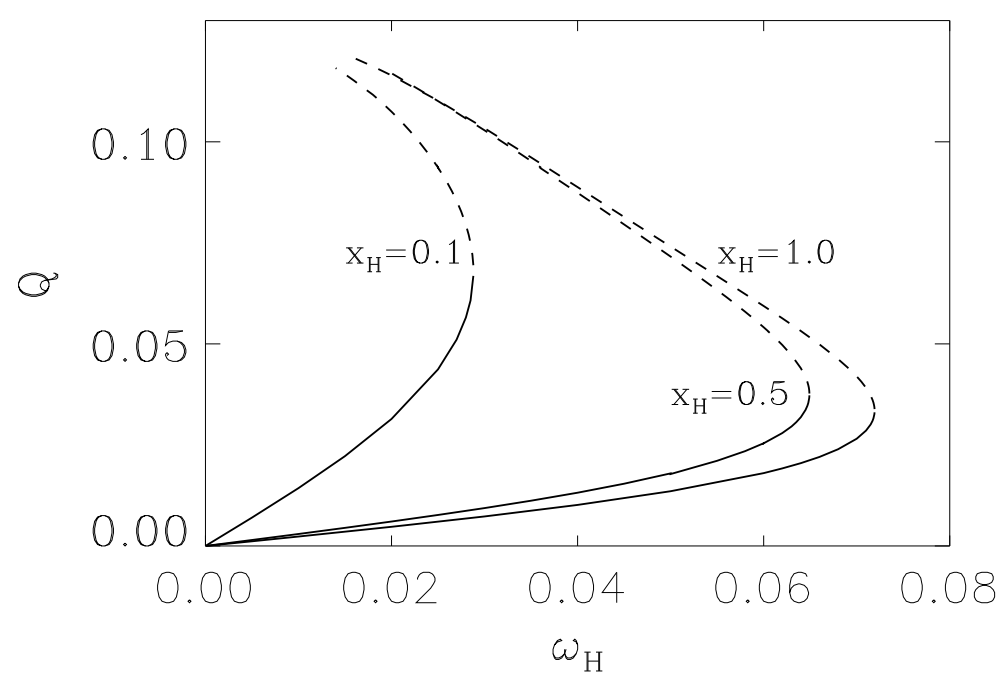

Figure 1c: The electric charge $Q$ is shown as a function of $\omega_{\mathrm{H}}$ for node number $k=1$ and $x_{\mathrm{H}}=1.0,0.5$ and 0.1 . 


\section{Fig.2}
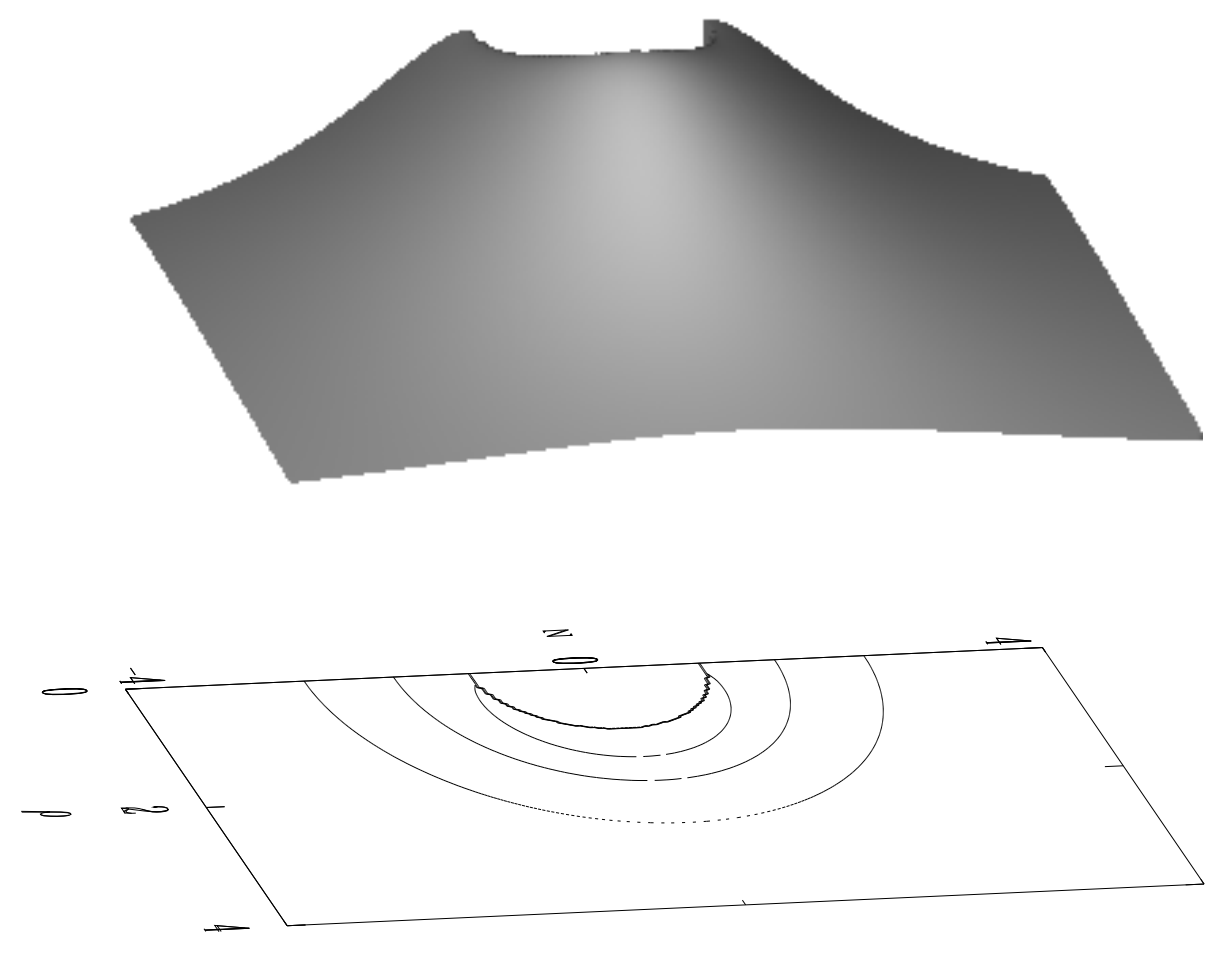

b

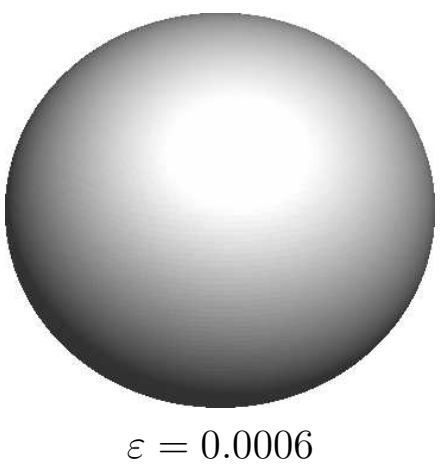

c

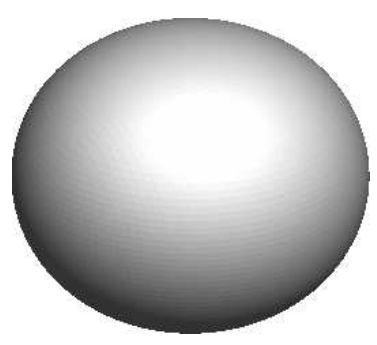

$\varepsilon=0.0009$ d

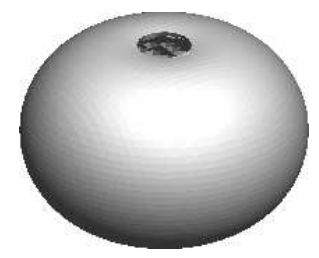

$\varepsilon=0.0011$

Figure 2: The energy density of the matter fields $\varepsilon=-T_{0}^{0}$ is shown as a funtion of the coordiantes $\rho=x \sin \theta, z=x \cos \theta$ for $k=1, x_{\mathrm{H}}=1.0, \omega_{\mathrm{H}}=0.04$ on the lower branch. 
Fig. 3
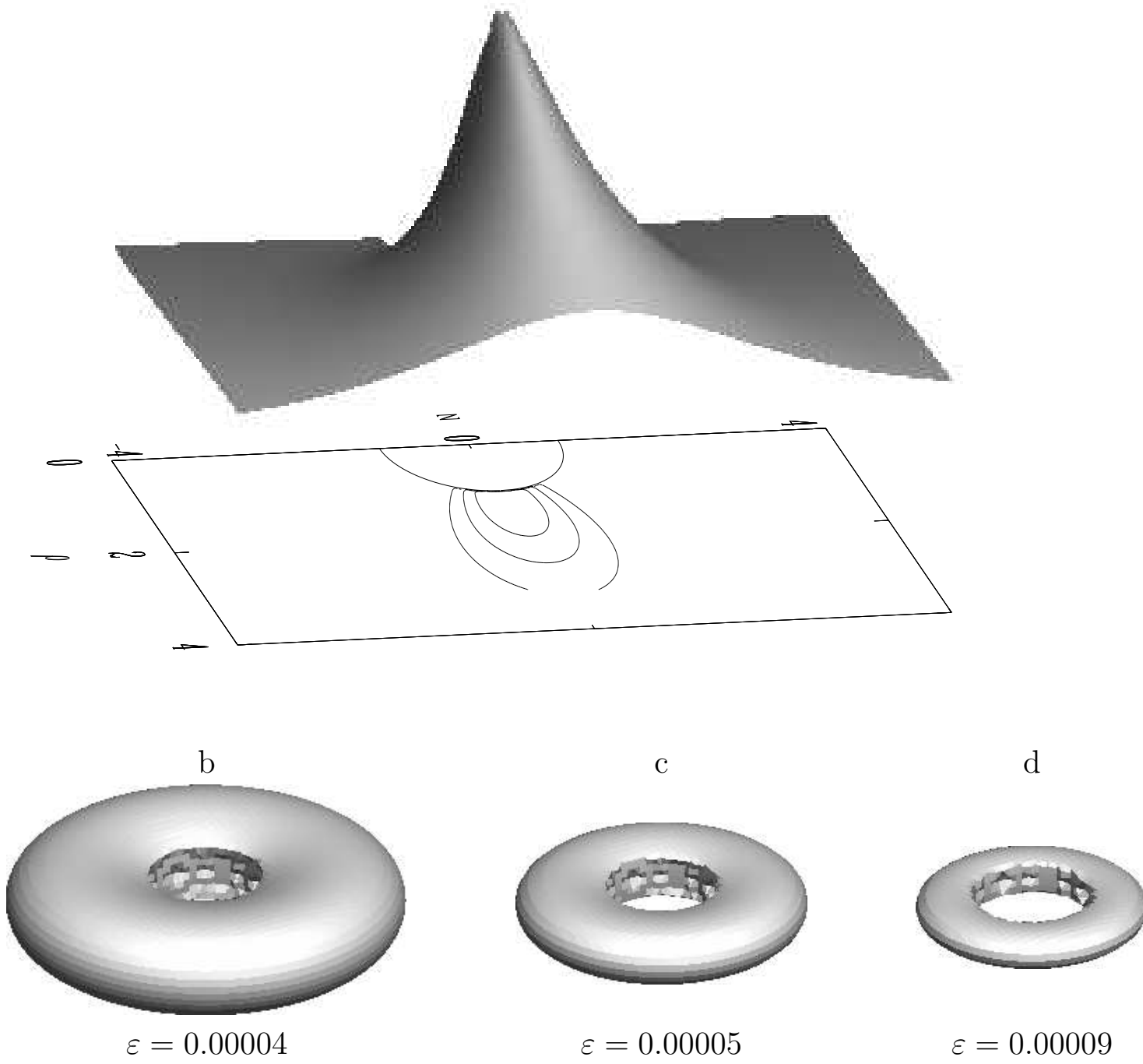

Figure 3: The same as Fig. 2 on the upper branch. 
Fig. $4 \mathrm{a}$

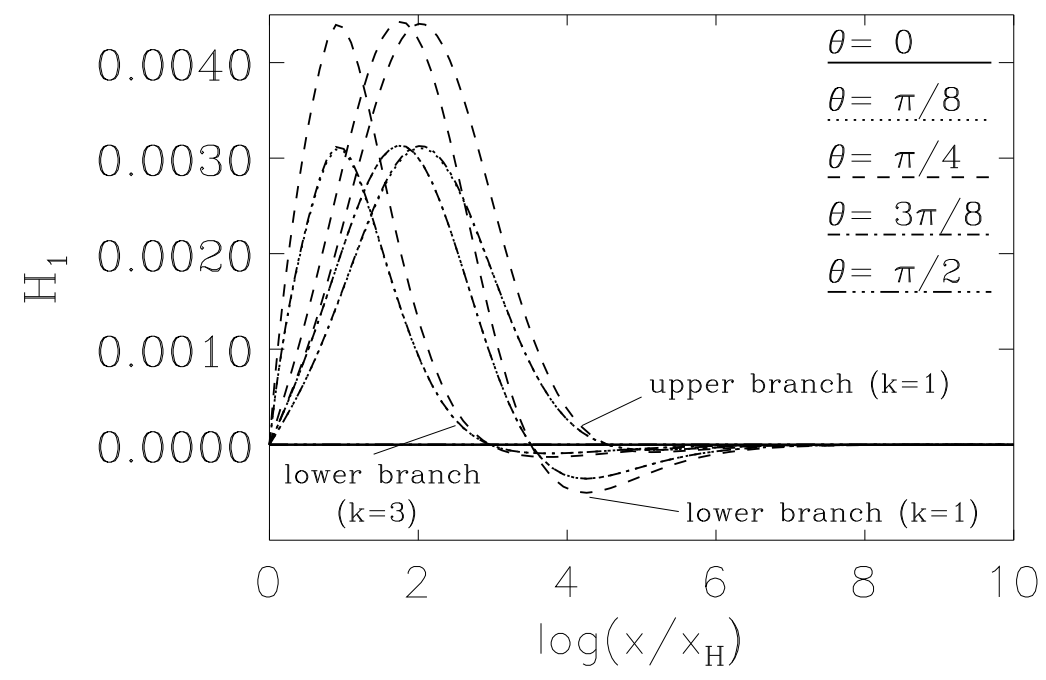

Figure 4a: The function $H_{1}$ is shown for $x_{\mathrm{H}}=1.0, \omega_{\mathrm{H}}=0.04$ and $k=1$ on the lower and upper branch, and for $k=3$ on the lower branch.

Fig. $4 b$

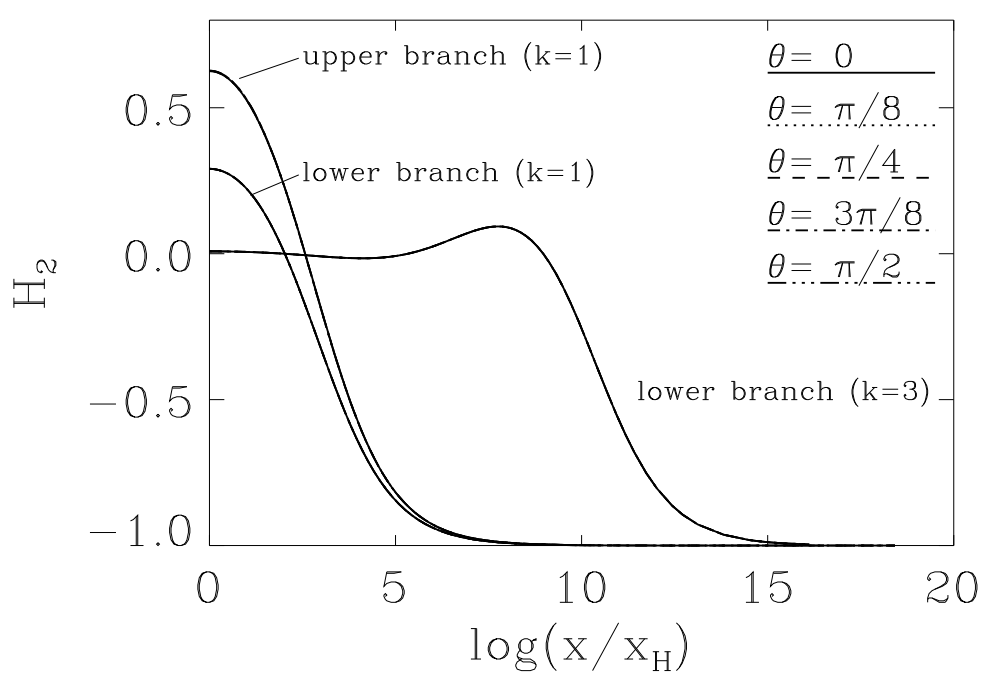

Figure 4b: Same as Fig. 4a for the function $H_{2}$. 
Fig. 4c

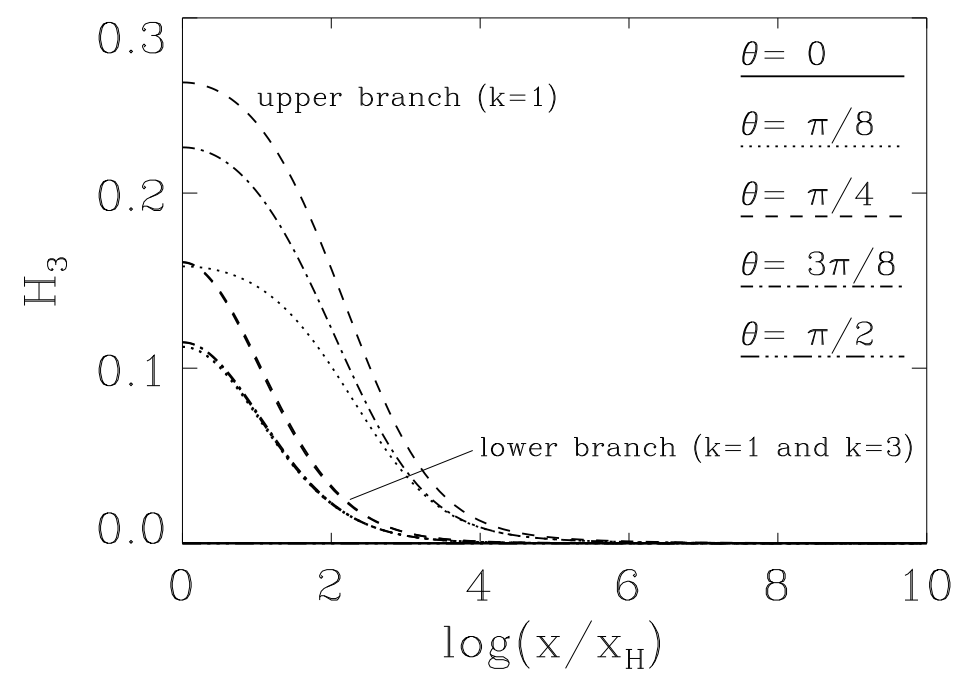

Figure 4c: Same as Fig. 4a for the function $H_{3}$. The curves for $k=1$ and $k=3$ on the lower branch coincide.

Fig. $4 \mathrm{~d}$

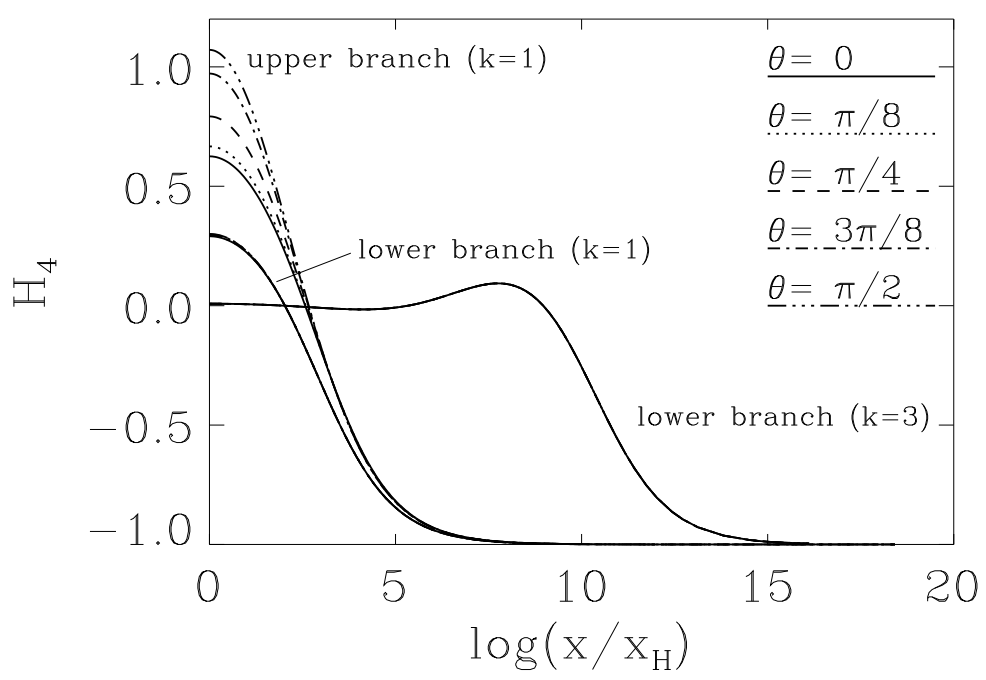

Figure 4d: Same as Fig. 5a for the function $H_{4}$. 
Fig. 4e

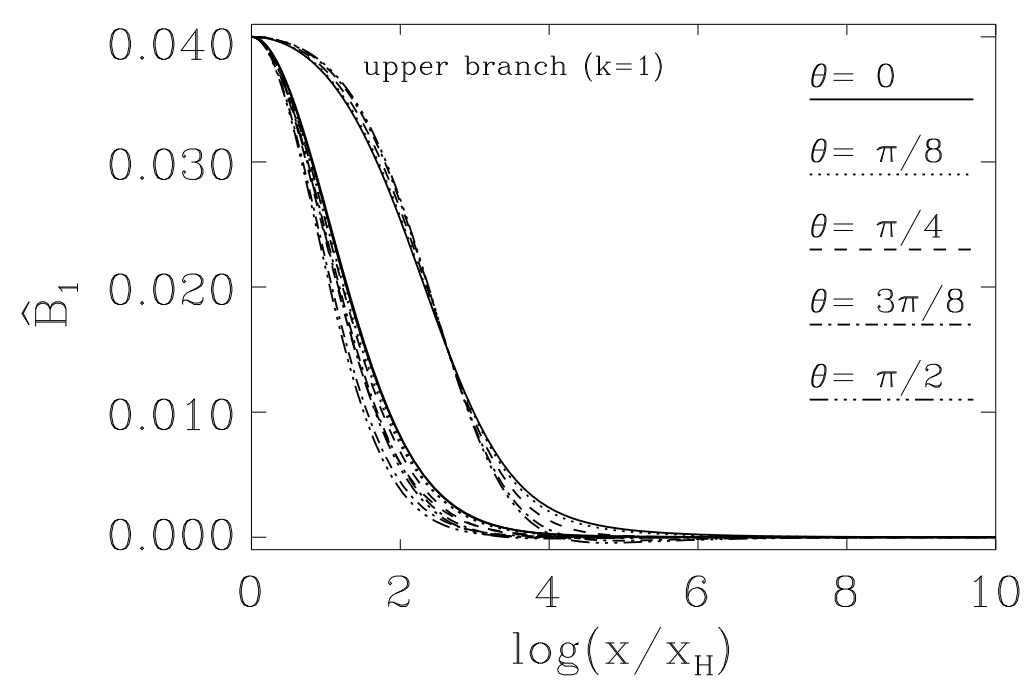

Figure 4e: Same as Fig. 4a for the function $\hat{B}_{1}$.

Fig. 4f

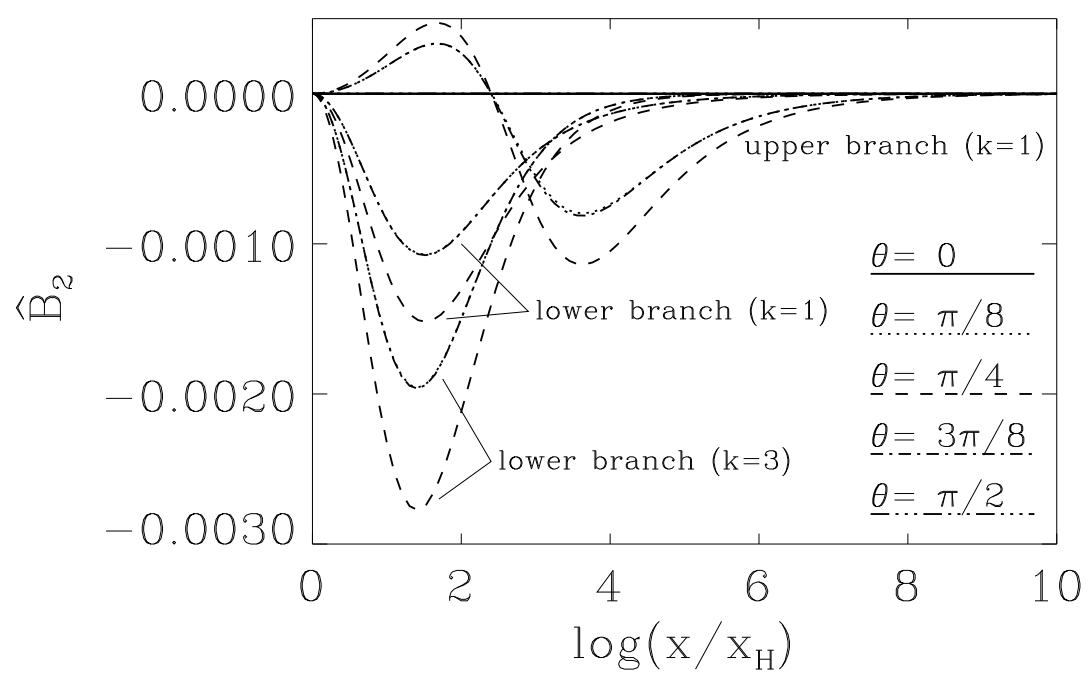

Figure 4f: Same as Fig. 4a for the function $\hat{B}_{2}$. 
Fig. $4 \mathrm{~g}$

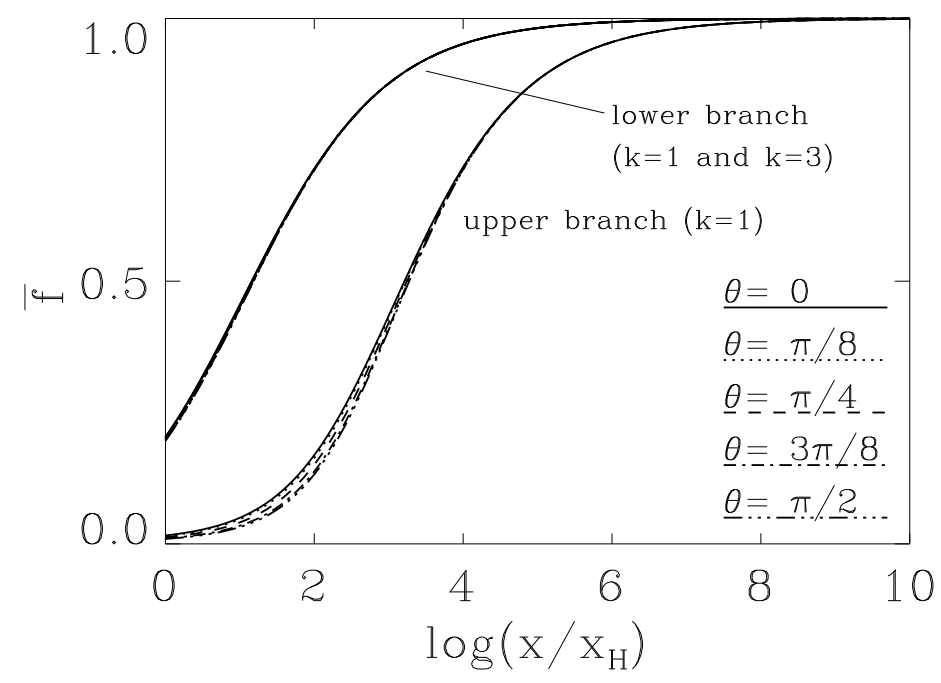

Figure 4g: Same as Fig. 4a for the function $\bar{f}$. The curves for $k=1$ and $k=3$ on the lower branch coincide.

Fig. $4 \mathrm{~h}$

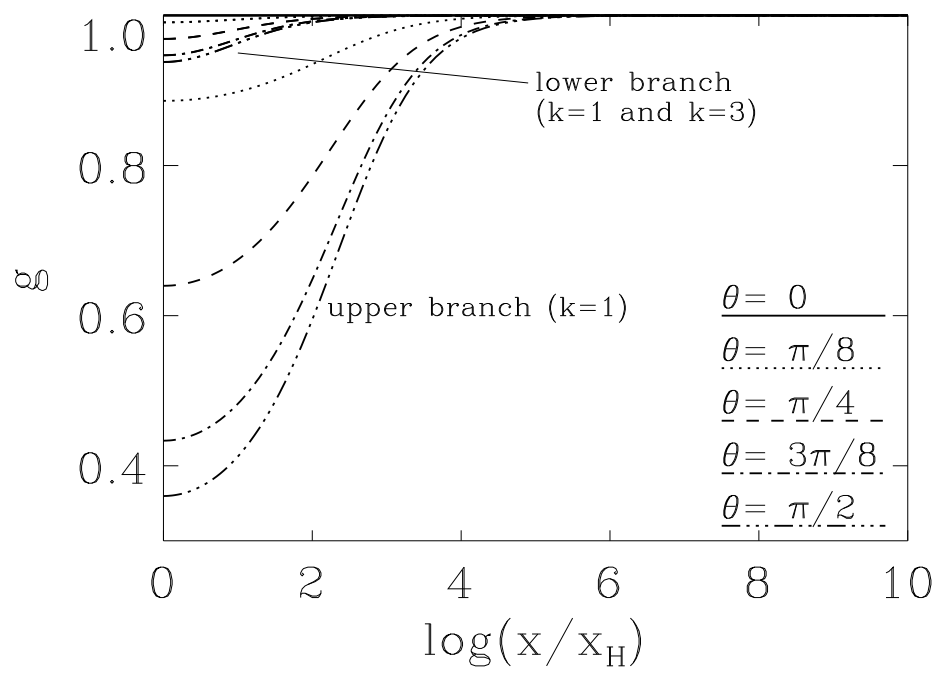

Figure 4h: Same as Fig. 4a for the function $g=m / l$. The curves for $k=1$ and $k=3$ on the lower branch coincide. 
Fig. 4i

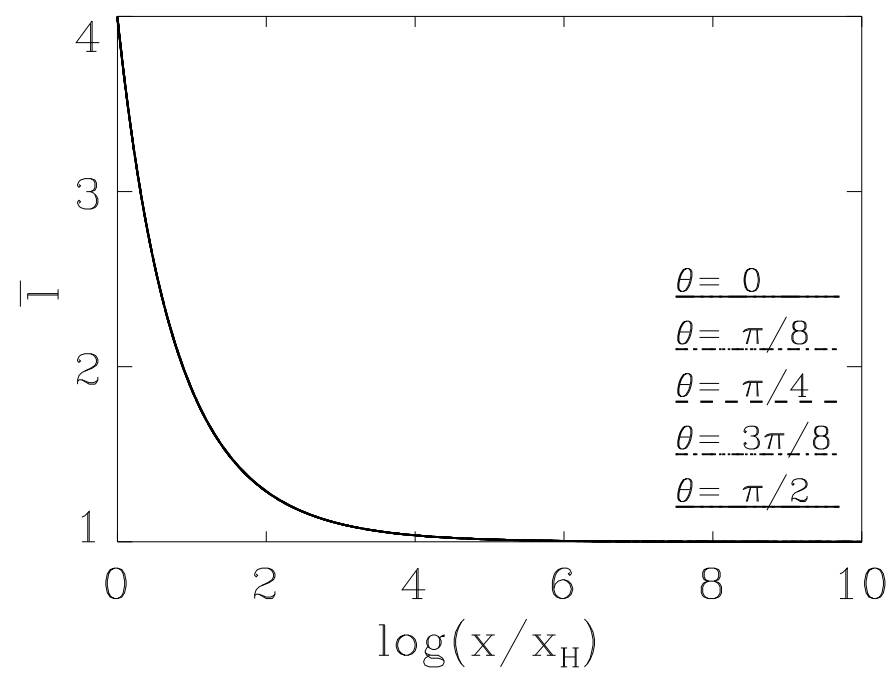

Figure 4i: Same as Fig. 4a for the function $\bar{l}$. The curves for $k=1$ and $k=3$ on the lower branch and $k=1$ on the upper branch coincide.

Fig. $4 j$

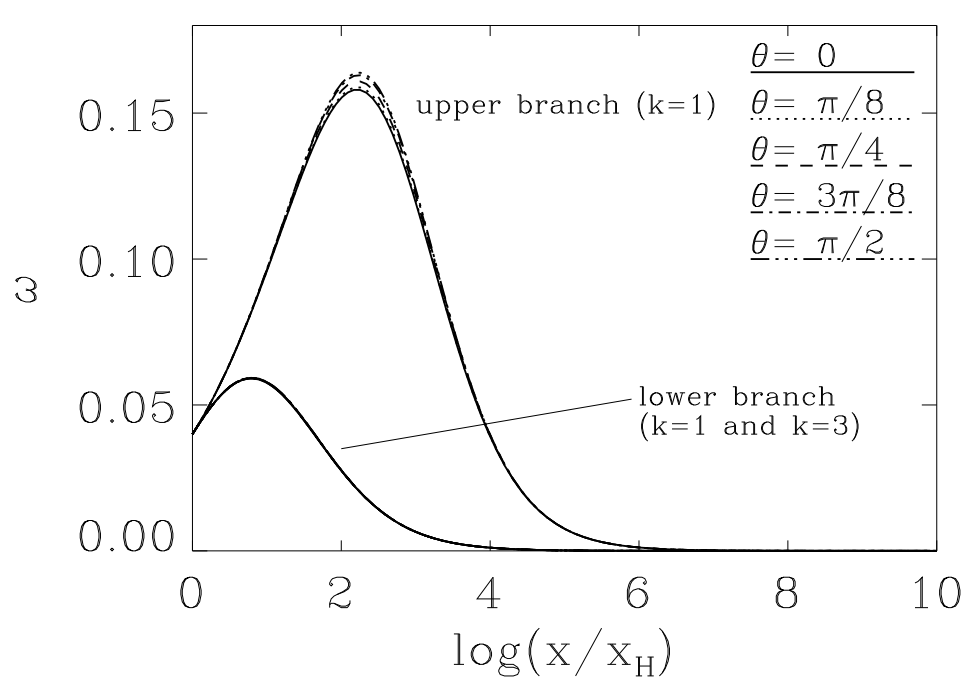

Figure 4j: Same as Fig. 4a for the function $\omega$. The curves for $k=1$ and $k=3$ on the lower branch coincide. 
Fig. 5a

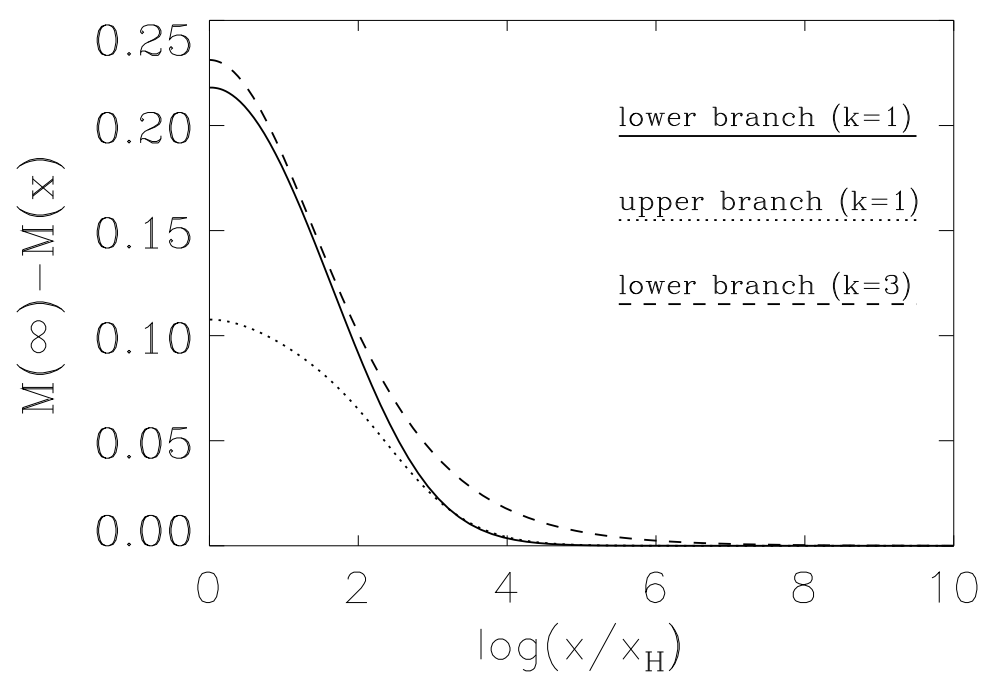

Figure 5a: The local mass $M(x)$ is shown for $x_{\mathrm{H}}=1.0, \omega_{\mathrm{H}}=0.04$ and $k=1$ on the lower and upper branch, and for $k=3$ on the lower branch.

Fig. 5b

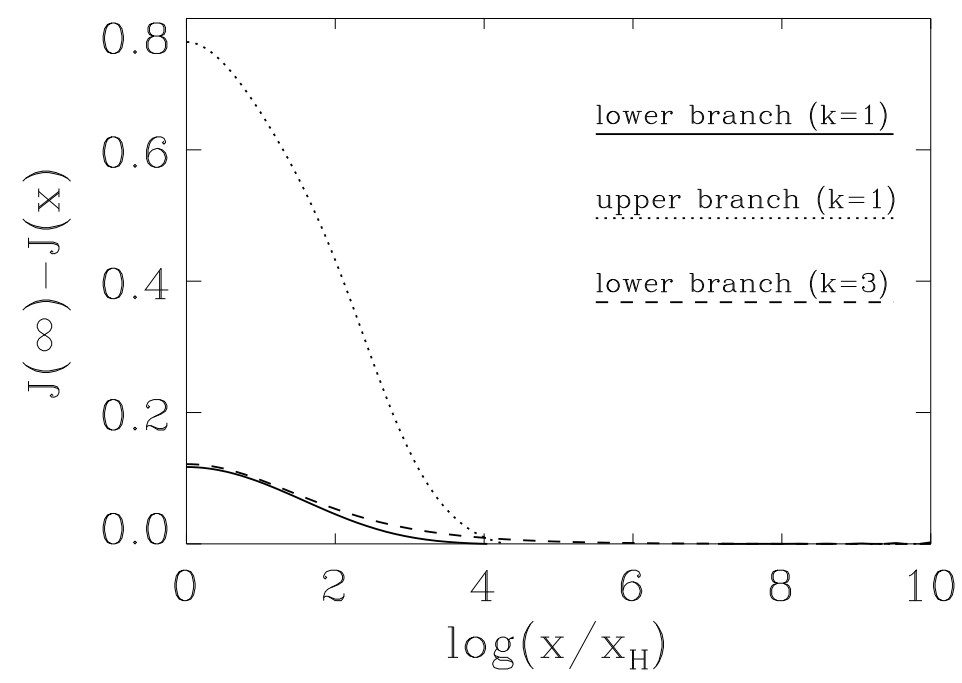

Figure 5b: The same as Fig. 5a for the local angular monetum $J(x)$. 
Fig. 5c

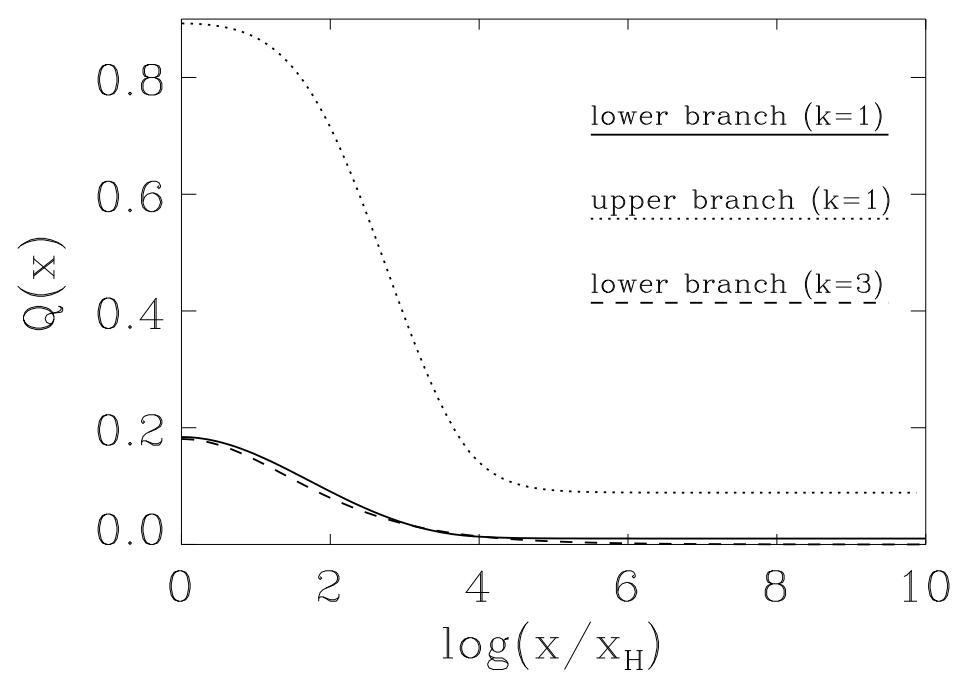

Figure 5c: The same as Fig. 5a for the local electric charge $Q(x)$.

Fig. 5d

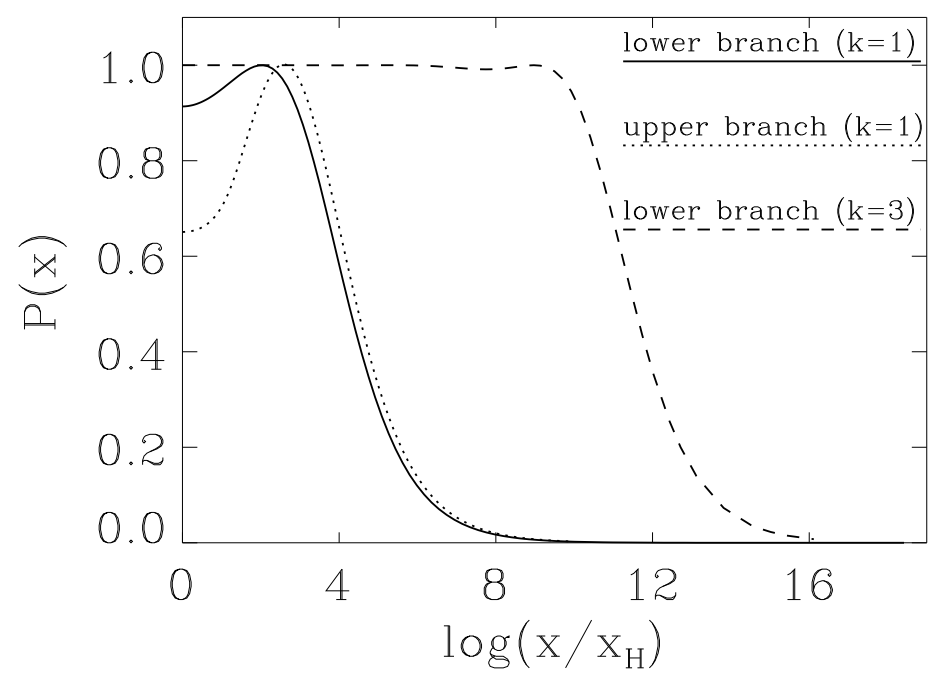

Figure 5d: The same as Fig. 5a for the local magnetic charge $P(x)$. 
Fig. 6a

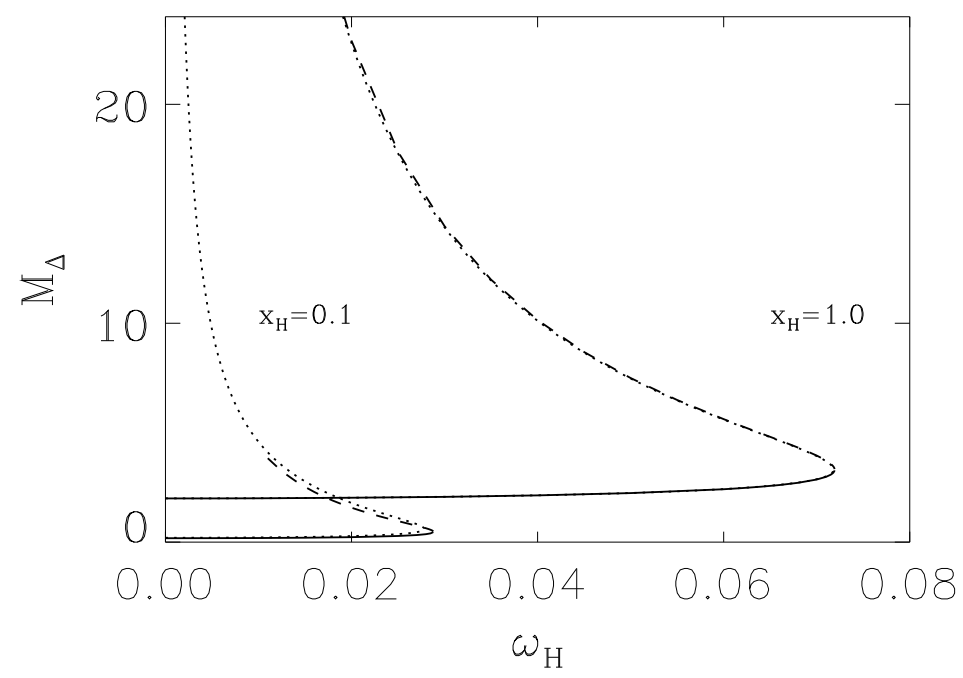

Figure 6a: The horizon mass $M_{\Delta}$ is shown as a function of $\omega_{\mathrm{H}}$ for $k=1, x_{\mathrm{H}}=1$ and $x_{\mathrm{H}}=0.1$ on the lower branch (solid) and on the upper branch (dashed). For the same values of parameters the corresponding functions of the Kerr solution (thin solid) and the Kerr-Newman solution (dotted) for $Q=0$ and $|P|=1$ are also shown. 
Fig. $6 \mathrm{~b}$

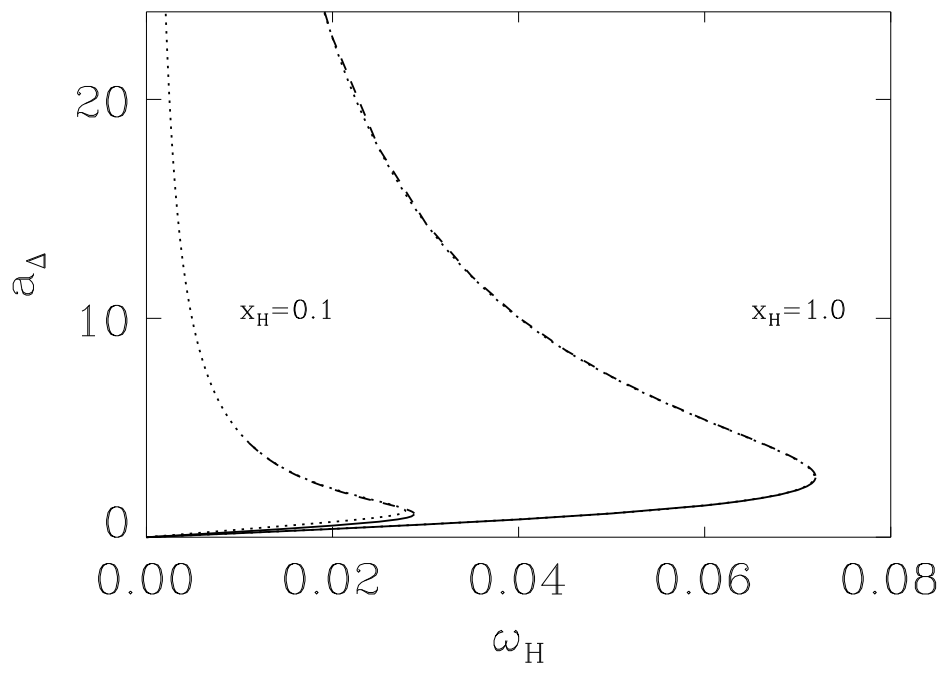

Figure 6b: The same as Fig. 6a for the angular momentum per mass at the horizon $a_{\Delta}=J_{\Delta} / M_{\Delta}$.

Fig. 6c

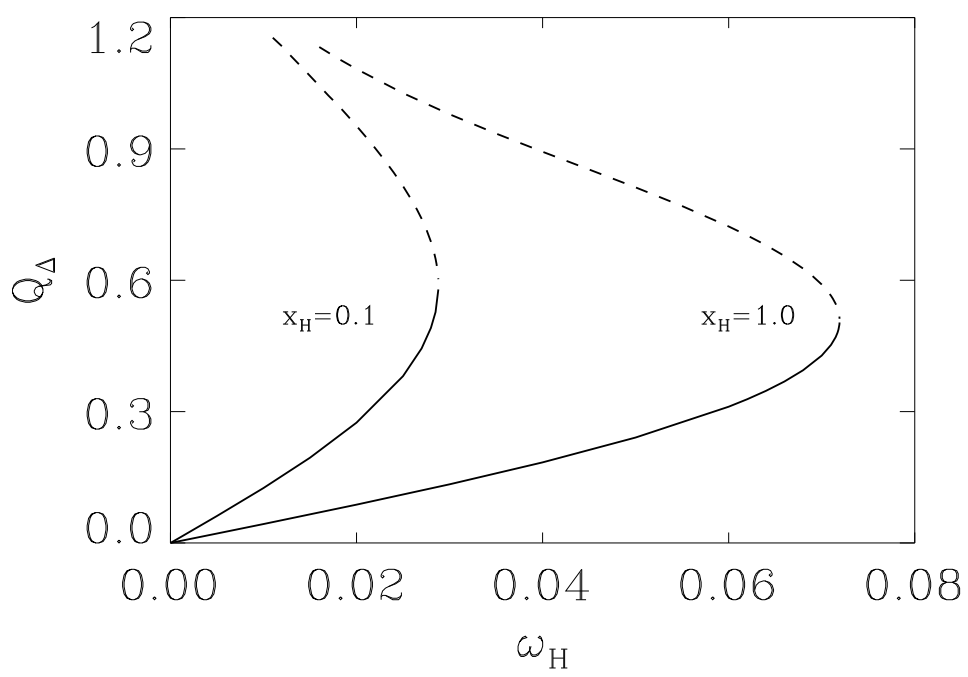

Figure 6c: The same as Fig. 6a for the horizon electric charge $Q_{\Delta}$. 
Fig. 6d

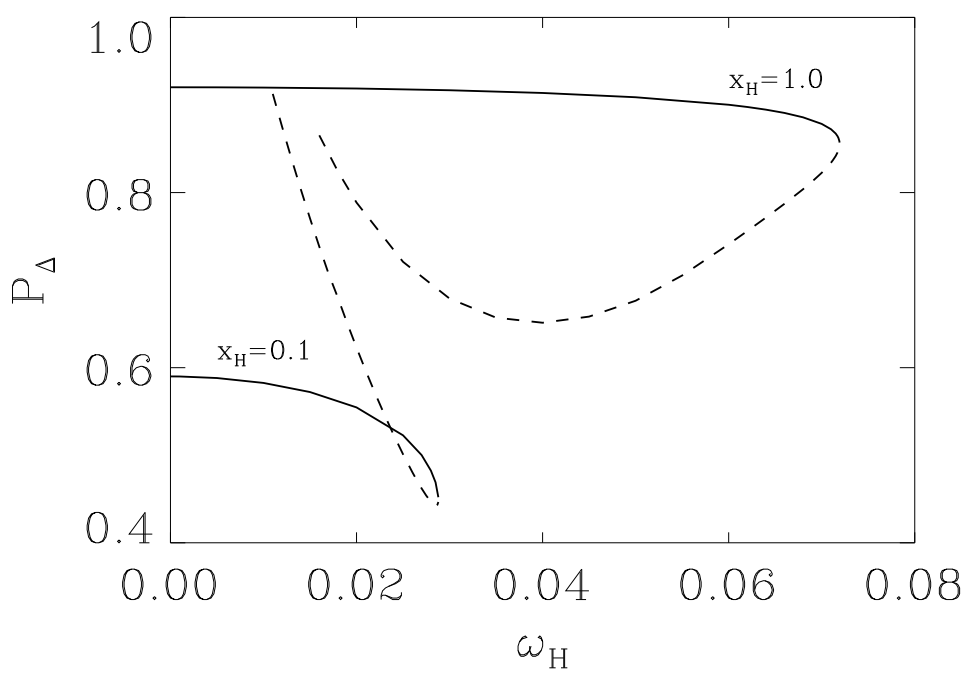

Figure 6d: The same as Fig. 6a for the horizon magnetic charge $P_{\Delta}$. 
Fig. $7 \mathrm{a}$

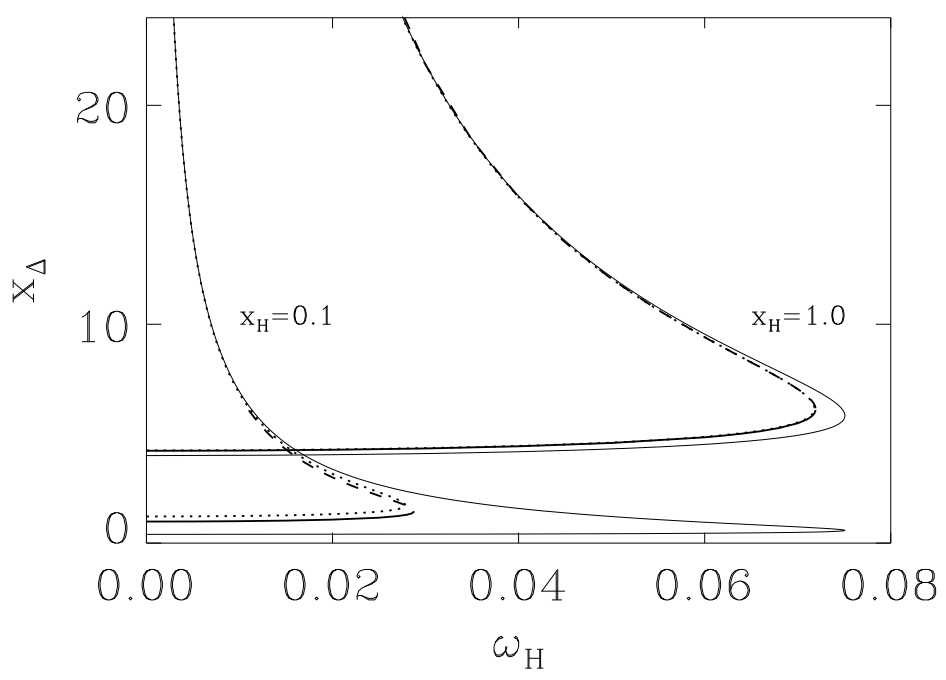

Figure 7a: The area parameter $x_{\Delta}$ is shown as a function of $\omega_{\mathrm{H}}$ for $k=1, x_{\mathrm{H}}=1$ and $x_{\mathrm{H}}=0.1$ on the lower branch (solid) and on the upper branch (dashed). For the same values of parameters the corresponding functions of the Kerr solution (thin solid) and the Kerr-Newman solution (dotted) for $Q=0$ and $|P|=1$ are also shown. 
Fig. $7 b$

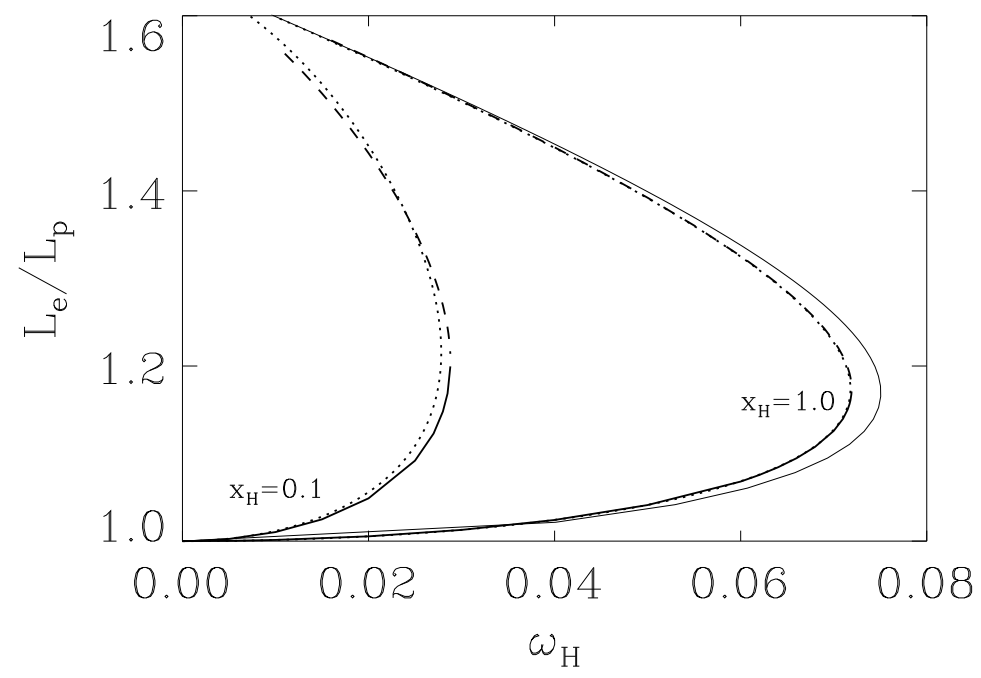

Figure 7b: The same as Fig. 7a for the horizon electric charge $Q_{\Delta}$.

Fig. 7c

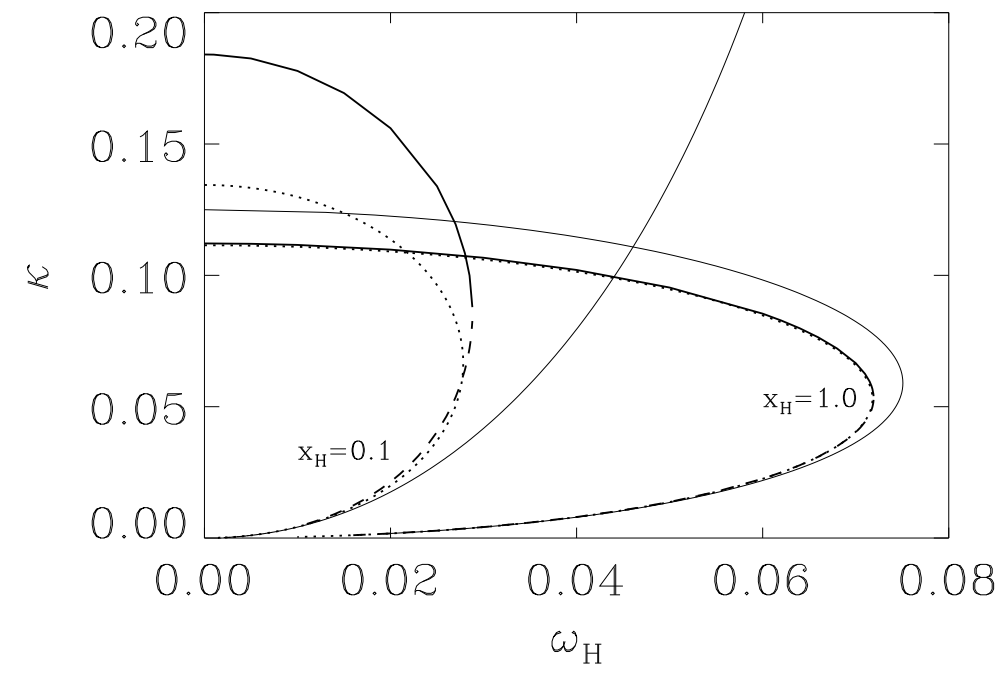

Figure 7c: The same as Fig. 7a for the surface gravity $\kappa$. 
Fig. 8

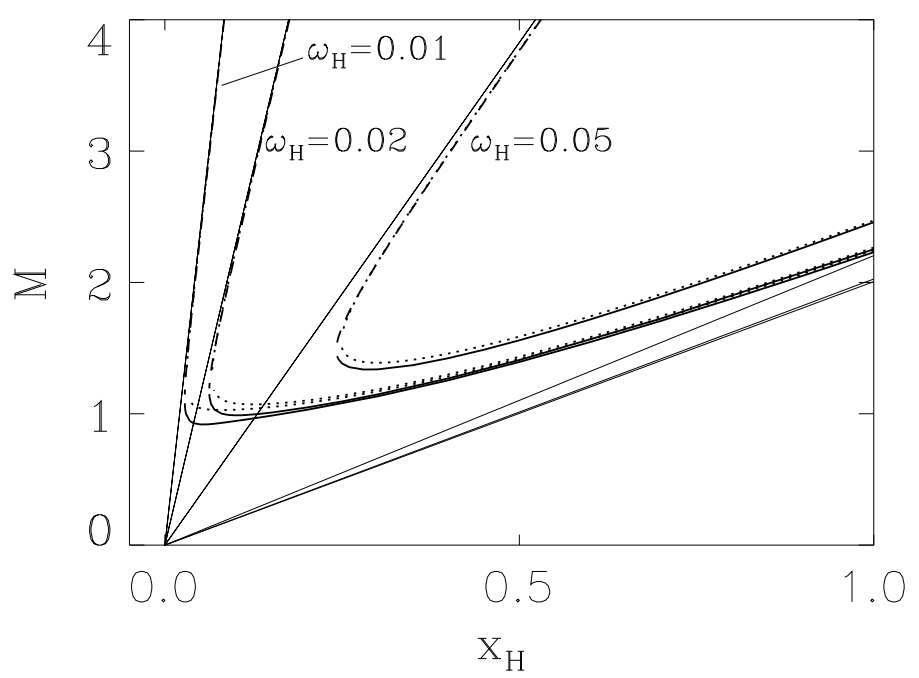

Figure 8: The dimensionless mass $M$ is shown as a function of $x_{\mathrm{H}}$ for $k=1$ and $\omega_{\mathrm{H}}=0.01,0.02$ and 0.05 on the lower branch (solid) and on the upper branch (dashed). For the same values of parameters the corresponding functions of the Kerr solution (thin solid) the Kerr-Newman solution (dotted) for $Q=0$ and $|P|=1$ are also shown. 
Fig. 9

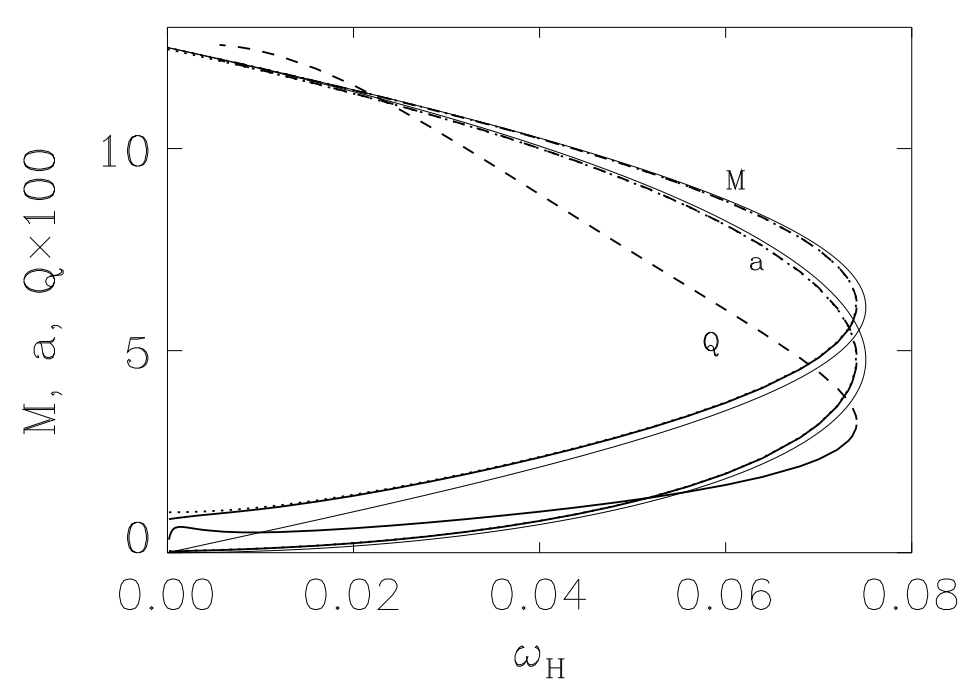

Figure 9: The dimensionless mass $M$, ratio $a=J / M$ and electric charge $Q$ are shown as function of $x_{\mathrm{H}}$ for $k=1$ and fixed $\omega_{\mathrm{H}} / x_{\mathrm{H}}=0.04$ on the lower branch (solid) and on the upper branch (dashed). For the same values of parameters the corresponding functions of the Kerr solution (thin solid) the Kerr-Newman solution (dotted) for $Q=0$ and $|P|=1$ are also shown. 
Fig. 10

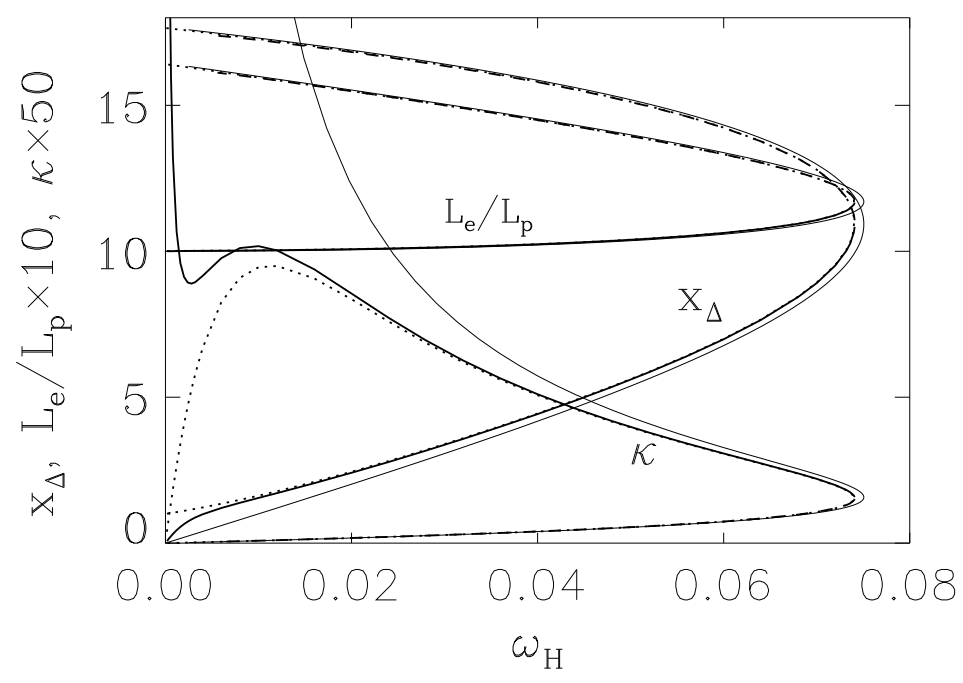

Figure 10: The same as Fig. 9 for the horizon parameter $x_{\Delta}$, the ratio $L_{e} / L_{p}$ and the surface curvature $\kappa$. 